\title{
AFLT-TYPE SELBERG INTEGRALS
}

\author{
SEAMUS P. ALBION, ERIC M. RAINS, AND S. OLE WARNAAR \\ Dedicated to the memory of Richard (Dick) Askey.
}

\begin{abstract}
In their 2011 paper on the AGT conjecture, Alba, Fateev, Litvinov and Tarnopolsky (AFLT) obtained a closed-form evaluation for a Selberg integral over the product of two Jack polynomials, thereby unifying the well-known Kadell and Hua-Kadell integrals. In this paper we use a variety of symmetric functions and symmetric function techniques to prove generalisations of the AFLT integral. These include (i) an $\mathrm{A}_{n}$ analogue of the AFLT integral, containing two Jack polynomials in the integrand; (ii) a generalisation of (i) for $\gamma=1$ (the Schur or GUE case), containing a product of $n+1$ Schur functions; (iii) an elliptic generalisation of the AFLT integral in which the role of the Jack polynomials is played by a pair of elliptic interpolation functions; (iv) an AFLT integral for Macdonald polynomials.
\end{abstract}

Keywords: AGT conjecture, (complex) Schur functions, elliptic beta integrals, elliptic interpolation functions, Jack polynomials, Macdonald polynomials, Selberg integrals.

\section{INTRODUCTION}

In 2010 Alday, Gaiotto and Tachikawa 3] conjectured a deep relationship between $\mathcal{N}=2$ superconformal field theory in four dimensions and Liouville conformal field theory on a punctured Riemann surface. Their correspondence provides a dictionary between correlation functions in Liouville field theory [36, 58] and the Nekrasov partition function in $\mathcal{N}=2$ superconformal field theory [37, 38]. One entry of this dictionary relates the instanton part of the Nekrasov partition function to conformal blocks in Liouville field theory. This relationship allowed Alday et al. to derive an explicit combinatorial expansion for conformal blocks.

One particularly promising approach to the AGT conjecture was developed by Alba, Fateev, Litvinov and Tarnopolsky [1]. Let Vir and $\mathscr{A}$ denote the Virasoro and Heisenberg algebras respectively. Then Alba et al. considered representations $L(P, Q)$ of central charge and conformal dimension

$$
c=1+6 Q^{2} \quad \text { and } \quad \Delta(P)=Q^{2} / 4-P^{2}
$$

of $\operatorname{Vir} \oplus \mathscr{A}$, and showed that $L(P, Q)$ has a unique orthogonal basis $\left\{\left|P_{\boldsymbol{\lambda}}\right\rangle\right\}$ indexed by bipartitions $\boldsymbol{\lambda}$, such that in this basis the matrix element between $L(P, Q)$ and $L\left(P^{\prime}, Q\right)$ corresponding to the primary field indexed by $\alpha$ coincides with

$$
Z_{\text {bifund }}\left(\left(P^{\prime},-P^{\prime}\right), \boldsymbol{\lambda} ;(P,-P), \boldsymbol{\mu} ; \alpha\right) \text {. }
$$

Here $Z_{\text {bifund }}$ is the key building block - corresponding to the 'bifundamental hypermultiplet' - of the instanton part of the Nekrasov partition function, which admits the following explicit combinatorial expression [13, 16, 52]

2010 Mathematics Subject Classification. 05E05, 05E10, 30E20, 33D05, 33D52, 33D67, 81T40.

Work supported by the Australian Research Council Discovery Grant DP170102648. 


$$
\begin{aligned}
Z_{\text {bifund }}\left(\left(u_{1}, u_{2}\right), \boldsymbol{\lambda} ;\left(v_{1}, v_{2}\right), \boldsymbol{\mu} ; m\right)=\prod_{i, j=1}^{2}( & \prod_{s \in \lambda^{(i)}}\left(E\left(u_{i}-v_{j}, \lambda^{(i)}, \mu^{(j)}, s\right)-m\right) \\
& \left.\times \prod_{s \in \mu^{(j)}}\left(Q-m-E\left(v_{j}-u_{i}, \mu^{(j)}, \lambda^{(i)}, s\right)\right)\right),
\end{aligned}
$$

where $\boldsymbol{\lambda}=\left(\lambda^{(1)}, \lambda^{(2)}\right), \boldsymbol{\mu}=\left(\mu^{(1)}, \mu^{(2)}\right)$ and, for $s=(i, j) \in \lambda$,

$$
E(u, \lambda, \mu, s)=u-b\left(\mu_{j}^{\prime}-i\right)+b^{-1}\left(\lambda_{i}-j+1\right)
$$

with $Q$ in (1.1) parametrised as $Q=b+b^{-1}$. In the above $\mu^{\prime}$ is the conjugate of the partition $\mu$, so that $\mu_{j}^{\prime}-i$ and $\lambda_{i}-j$ may be recognised as the (generalised) leg-length $l_{\mu}(s)$ and arm-length $a_{\lambda}(s)$ of the square $s \in \lambda$, see (2.1). Lifting the isomorphism [12, 59] between Verma modules for Vir and Fock space representations of $\mathscr{A}$ to the level of $L(P, Q)$, Alba et al. obtained a closed-form expression in the spirit of [34, 50, 65] for the states $|P\rangle_{\lambda, 0}$ in terms of Jack polynomials $P_{\lambda}^{\left(-1 / b^{2}\right)}$. The more general states $|P\rangle_{\boldsymbol{\lambda}}$ then follow recursively from $|P\rangle_{\lambda, 0}$.

For $\mathscr{O}$ a symmetric Laurent polynomial in $k$ variables and $\alpha, \beta, \gamma \in \mathbb{C}$, define the Selberg average of $\mathscr{O}$ as

$$
\langle\mathscr{O}\rangle_{\alpha, \beta ; \gamma}^{k}:=\frac{1}{S_{k}(\alpha, \beta ; \gamma)} \int_{[0,1]^{k}} \mathscr{O}\left(t_{1}, \ldots, t_{k}\right) \prod_{i=1}^{k} t_{i}^{\alpha-1}\left(1-t_{i}\right)^{\beta-1} \prod_{1 \leqslant i<j \leqslant k}\left|t_{i}-t_{j}\right|^{2 \gamma} \mathrm{d} t_{1} \cdots \mathrm{d} t_{k},
$$

where the normalisation $S_{k}(\alpha, \beta ; \gamma)$ is given by the classical Selberg integral [14, 15, 51, 57]

$$
\begin{aligned}
S_{k}(\alpha, \beta ; \gamma) & :=\int_{[0,1]^{k}} \prod_{i=1}^{k} t_{i}^{\alpha-1}\left(1-t_{i}\right)^{\beta-1} \prod_{1 \leqslant i<j \leqslant k}\left|t_{i}-t_{j}\right|^{2 \gamma} \mathrm{d} t_{1} \cdots \mathrm{d} t_{k} \\
& =\prod_{i=1}^{k} \frac{\Gamma(\beta+(i-1) \gamma) \Gamma(\alpha+(i-1) \gamma) \Gamma(1+i \gamma)}{\Gamma(\alpha+\beta+(2 k-i-1) \gamma) \Gamma(1+\gamma)},
\end{aligned}
$$

for $\operatorname{Re}(\alpha)>0, \operatorname{Re}(\beta)>0$ and

$$
\operatorname{Re}(\gamma)>-\min \{1 / k, \operatorname{Re}(\alpha) /(k-1), \operatorname{Re}(\beta) /(k-1)\} .
$$

A crucial step taken by Alba et al. was to show that for $P+P^{\prime}+\alpha+k b=0$

$$
\begin{aligned}
Z_{\mathrm{bifund}} & \left(\left(P^{\prime},-P^{\prime}\right),(\lambda, 0) ;(P,-P),(\mu, 0) ; \alpha\right) \\
& =\kappa_{\lambda}(P) \kappa_{\mu}\left(P^{\prime}\right)\left\langle P_{\lambda}^{\left(-1 / b^{2}\right)}\left(t^{-1}\right) P_{\mu}^{\left(-1 / b^{2}\right)}\left[t+\left(2 b \alpha-1-b^{2}\right) / b^{2}\right]\right\rangle_{1-b(Q+2 P), 1-2 b \alpha ;-b^{2}}^{k} .
\end{aligned}
$$

In the above,

$$
\kappa_{\lambda}(P):=\prod_{(i, j) \in \lambda}\left(\left(b\left(i-\lambda_{j}^{\prime}-1\right)+b^{-1}\left(\lambda_{i}-j\right)\right)\left(2 P+b i+b^{-1} j\right)\right)
$$


$f\left(t^{-1}\right):=f\left(t_{1}^{-1}, \ldots, t_{k}^{-1}\right)$ and, for $f$ a symmetric function which expands in terms of the power sums $p_{\lambda}$ as $f=\sum_{\lambda} c_{\lambda} p_{\lambda}, f[t+z]$ is plethystic notation (see Section 2.4 for details) for

$$
\sum_{\lambda} c_{\lambda} \prod_{i=1}^{l(\lambda)}\left(p_{\lambda_{i}}\left(t_{1}, \ldots, t_{k}\right)+z\right) .
$$

The verification of (1.5) boils down to computing the Selberg average

$$
\left\langle P_{\lambda}^{(1 / \gamma)}\left(t^{-1}\right) P_{\mu}^{(1 / \gamma)}[t+\beta / \gamma-1]\right\rangle_{\alpha, \beta ; \gamma}^{k}
$$

and comparing this against the explicit form of $Z_{\text {bifund }}$ provided by (1.2). By the complementation symmetry

$$
P_{\lambda}^{(1 / \gamma)}\left(t^{-1}\right)=\left(t_{1} \cdots t_{k}\right)^{-N} P_{\left(N-\lambda_{k}, \ldots, N-\lambda_{1}\right)}^{(1 / \gamma)}(t),
$$

for $\lambda \in \mathscr{P}_{k}$ (the set of partitions of length at most $k$ ) and $N$ an arbitrary integer such that $N \geqslant \lambda_{1}$, this is achieved by the following integral evaluation [1] (see also [35]). Let $\mathscr{P}$ denote the set of partitions.

Theorem 1.1 (AFLT integral). Let $k$ be a positive integer, $\lambda, \mu \in \mathscr{P}$ and $\alpha, \beta, \gamma \in \mathbb{C}$. Then

$$
\begin{aligned}
& \int_{[0,1]^{k}} P_{\lambda}^{(1 / \gamma)}[t] P_{\mu}^{(1 / \gamma)}[t+\beta / \gamma-1] \prod_{i=1}^{k} t_{i}^{\alpha-1}\left(1-t_{i}\right)^{\beta-1} \prod_{1 \leqslant i<j \leqslant k}\left|t_{i}-t_{j}\right|^{2 \gamma} \mathrm{d} t_{1} \cdots \mathrm{d} t_{k} \\
& =P_{\lambda}^{(1 / \gamma)}[k] P_{\mu}^{(1 / \gamma)}[k+\beta / \gamma-1] \prod_{i=1}^{k} \frac{\Gamma(\beta+(i-1) \gamma) \Gamma\left(\alpha+(k-i) \gamma+\lambda_{i}\right) \Gamma(1+i \gamma)}{\Gamma\left(\alpha+\beta+(2 k-m-i-1) \gamma+\lambda_{i}\right) \Gamma(1+\gamma)} \\
& \quad \times \prod_{i=1}^{k} \prod_{j=1}^{m} \frac{\Gamma\left(\alpha+\beta+(2 k-i-j-1) \gamma+\lambda_{i}+\mu_{j}\right)}{\Gamma\left(\alpha+\beta+(2 k-i-j) \gamma+\lambda_{i}+\mu_{j}\right)}
\end{aligned}
$$

where $m$ is an arbitrary integer such that $m \geqslant l(\mu)$, and

$$
\operatorname{Re}(\alpha)>-\lambda_{k}, \quad \operatorname{Re}(\beta)>0, \quad \operatorname{Re}(\gamma)>-\min _{1 \leqslant i \leqslant k-1}\left\{\frac{1}{k}, \frac{\operatorname{Re}(\alpha)+\lambda_{i}}{k-i}, \frac{\operatorname{Re}(\beta)}{k-1}\right\} .
$$

The arguments of the Jack polynomials on the right-hand side are again expressed using plethystic notation, see Section 2.4 for details. Alternatively, both $P_{\lambda}^{(1 / \gamma)}[k]$ and $P_{\mu}^{(1 / \gamma)}[k+\beta / \gamma-1]$ may be written in fully factorised form by equation (2.20) below. When $\mu=0$ the AFLT integral simplifies to Kadell's integral [24] (see also [31]) and for $\beta=\gamma$ it yields the Hua-Kadell integral [19, 23]. Theorem 1.1 is proved in [1] by generalising the Anderson-style recursive proof of Kadell's integral given in [61. Key input in both these proofs is the Okounkov-Olshanski integral formula for Jack polynomials [39, 41].

In the conclusion to their paper [1, Alba et al. remark that the generalisation of their construction to $\mathrm{WA}_{n}$ requires a generalisation of the $\mathrm{A}_{n}$ Selberg integral of 63. with two Jack polynomials included in the integrand 1$]$ For $\mathrm{A}_{2}$ such an AFLT-type integral was considered by Fateev and Litvinov in [11, where they again used a recursion based on the Okounkov-Olshanski integral to obtain a closed-form evaluation. They also claim a more general $\mathrm{A}_{n}$ AFLT integral, but the

\footnotetext{
${ }^{1}$ See also [7, 22] for the relation between $\mathrm{A}_{n}$ Selberg integrals and the AGT conjecture.
} 
evaluation of this integral is only implicit and, unfortunately, the stated recursion relation that needs to be solved to obtain the evaluation is incorrect for $n \geqslant 3$ (see also (7.4) in Section (7).

The first main result of the present paper is the evaluation of the $\mathrm{A}_{n}$ AFLT integral for all $n$. Before stating this result we introduce some notation. For $k, \ell$ nonnegative integers and $t=$ $\left(t_{1}, \ldots, t_{k}\right), s=\left(s_{1}, \ldots, s_{\ell}\right)$ we define the Vandermonde products $\Delta(t)$ and $\Delta(t, s)$ as

$$
\Delta(t):=\prod_{1 \leqslant i<j \leqslant k}\left(t_{i}-t_{j}\right) \text { and } \Delta(t, s):=\prod_{i=1}^{k} \prod_{j=1}^{\ell}\left(t_{i}-s_{j}\right) .
$$

We also abbreviate $\mathrm{d} t_{1} \cdots \mathrm{d} t_{k}$ as $\mathrm{d} t$.

For $n$ a positive integer, let $k_{1}, \ldots, k_{n}$ be integers such that $0 \leqslant k_{1} \leqslant \cdots \leqslant k_{n}$ and let $t^{(1)}, \ldots, t^{(n)}$ be a sets of variables (or alphabets) such that $t^{(r)}$ has cardinality $k_{r}$. Further let $\alpha_{1}, \ldots, \alpha_{n}, \beta \in \mathbb{C}$ such that

$$
\begin{gathered}
\operatorname{Re}(\beta)>0, \quad|\operatorname{Re}(\gamma)|<\frac{1}{k_{n}}, \quad \operatorname{Re}\left(\beta+\left(k_{n}-1\right) \gamma\right)>0, \\
\operatorname{Re}\left(\alpha_{r}+\cdots+\alpha_{s}+(r-s+i-1) \gamma\right)>0 \quad \text { for } 1 \leqslant r \leqslant s \leqslant n \text { and } 1 \leqslant i \leqslant k_{r}-k_{r-1},
\end{gathered}
$$

where $k_{0}:=0.2$ We then define the $\mathrm{A}_{n}$ Selberg average of a polynomial $\mathscr{O}\left(t^{(1)}, \ldots, t^{(n)}\right)$, symmetric in each of the alphabets $t^{(r)}$, as

$$
\langle\mathscr{O}\rangle_{\alpha_{1}, \ldots, \alpha_{n}, \beta ; \gamma}^{k_{1}, \ldots, k_{n}}:=\frac{I_{k_{1}, \ldots, k_{n}}^{\mathrm{A}_{n}}\left(\mathscr{O} ; \alpha_{1}, \ldots, \alpha_{n}, \beta ; \gamma\right)}{I_{k_{1}, \ldots, k_{n}}^{\mathrm{A}_{n}}\left(1 ; \alpha_{1}, \ldots, \alpha_{n}, \beta ; \gamma\right)},
$$

where

$$
\begin{aligned}
& I_{k_{1}, \ldots, k_{n}}^{\mathrm{A}_{n}}\left(\mathscr{O} ; \alpha_{1}, \ldots, \alpha_{n}, \beta ; \gamma\right) \\
&:=\int_{C_{\gamma}^{k_{1}, \ldots, k_{n}}[0,1]} \mathscr{O}\left(t^{(1)}, \ldots, t^{(n)}\right) \prod_{r=1}^{n} \prod_{i=1}^{k_{r}}\left(t_{i}^{(r)}\right)^{\alpha_{r}-1}\left(1-t_{i}^{(r)}\right)^{\beta_{r}-1} \\
& \quad \times \prod_{r=1}^{n}\left|\Delta\left(t^{(r)}\right)\right|^{2 \gamma} \prod_{r=1}^{n-1}\left|\Delta\left(t^{(r)}, t^{(r+1)}\right)\right|^{-\gamma} \mathrm{d} t^{(1)} \cdots \mathrm{d} t^{(n)} .
\end{aligned}
$$

Here

$$
\beta_{1}=\cdots=\beta_{n-1}:=1, \quad \beta_{n}:=\beta
$$

and $C_{\gamma}^{k_{1}, \ldots, k_{n}}[0,1]$ is a real domain of integration described in Section 4.1. The normalisation $I_{k_{1}, \ldots, k_{n}}^{\mathrm{A}_{n}}\left(1 ; \alpha_{1}, \ldots, \alpha_{n}, \beta ; \gamma\right)$ in (1.9) is the $\mathrm{A}_{n}$ Selberg integral of [63, Theorem 1.2] (see also [56, Theorem 3.3] for the $A_{2}$ case), which admits the evaluation

$$
\begin{aligned}
& I_{k_{1}, \ldots, k_{n}}^{\mathrm{A}_{n}}\left(1 ; \alpha_{1}, \ldots, \alpha_{n}, \beta ; \gamma\right) \\
& \quad=\prod_{r=1}^{n} \prod_{i=1}^{k_{r}} \frac{\Gamma\left(\beta_{r}+\left(i-k_{r+1}-1\right) \gamma\right) \Gamma(i \gamma)}{\Gamma(\gamma)}
\end{aligned}
$$

\footnotetext{
${ }^{2}$ The condition $\operatorname{Re}(\gamma)<1 / k_{n}$ may be dropped when $n=1$.
} 


$$
\times \prod_{1 \leqslant r \leqslant s \leqslant n} \prod_{i=1}^{k_{r}-k_{r-1}} \frac{\Gamma\left(\alpha_{r}+\cdots+\alpha_{s}+(r-s+i-1) \gamma\right)}{\Gamma\left(\alpha_{r}+\cdots+\alpha_{s}+\beta_{s}+\left(k_{s}-k_{s+1}+r-s+i-2\right) \gamma\right)},
$$

where, once again, $k_{0}=k_{n+1}:=0$.

For $n$ a nonnegative integer let $(a)_{n}:=\Gamma(a+n) / \Gamma(a)=a(a+1) \cdots(a+n-1)$ denote the Pochhammer symbol, and let $\delta_{u, v}$ be the usual Kronecker delta. Then the $\mathrm{A}_{n}$ analogue of the AFLT integral is given by the following identity for the Selberg average of the product of two Jack polynomials.

Theorem $1.2\left(\mathrm{~A}_{n}\right.$ AFLT integral). For $n$ a positive integer, let $k_{1}, \ldots, k_{n}$ be integers such that $0 \leqslant k_{1} \leqslant \cdots \leqslant k_{n}$. Then for $\alpha_{1}, \ldots, \alpha_{n}, \beta, \gamma \in \mathbb{C}$ such that (1.8) holds and $\lambda, \mu \in \mathscr{P}$, we have

$$
\begin{aligned}
\left\langle P_{\lambda}^{(1 / \gamma)}\right. & {\left.\left[t^{(1)}\right] P_{\mu}^{(1 / \gamma)}\left[t^{(n)}+\beta / \gamma-1\right]\right\rangle_{\alpha_{1}, \ldots, \alpha_{n}, \beta ; \gamma}^{k_{1}, \ldots, k_{n}} } \\
= & P_{\lambda}^{(1 / \gamma)}\left[k_{1}\right] P_{\mu}^{(1 / \gamma)}\left[k_{n}+\beta / \gamma-1\right] \\
& \times \prod_{r=1}^{n} \prod_{i=1}^{\ell} \frac{\left(\alpha_{1}+\cdots+\alpha_{r}+\left(k_{1}-r-i+1\right) \gamma\right)_{\lambda_{i}}}{\left(\alpha_{1}+\cdots+\alpha_{r}+\beta_{r}+\left(k_{1}+k_{r}-k_{r+1}-r-m \delta_{r, n}-i\right) \gamma\right)_{\lambda_{i}}} \\
& \times \prod_{r=1}^{n} \prod_{j=1}^{m} \frac{\left(\alpha_{r}+\cdots+\alpha_{n}+\beta+\left(k_{n}+r-n-j-1\right) \gamma\right)_{\mu_{j}}}{\left(\alpha_{r}+\cdots+\alpha_{n}+\beta+\left(k_{r}-k_{r-1}+k_{n}+r-n-\ell \delta_{r, 1}-j-1\right) \gamma\right)_{\mu_{j}}} \\
& \times \prod_{i=1}^{\ell} \prod_{j=1}^{m} \frac{\left(\alpha_{1}+\cdots+\alpha_{n}+\beta+\left(k_{1}+k_{n}-n-i-j\right) \gamma\right)_{\lambda_{i}+\mu_{j}}}{\left(\alpha_{1}+\cdots+\alpha_{n}+\beta+\left(k_{1}+k_{n}-n-i-j+1\right) \gamma\right)_{\lambda_{i}+\mu_{j}}} .
\end{aligned}
$$

In the expression on the right, $\ell$ and $m$ are arbitrary integers such that $\ell \geqslant l(\lambda), m \geqslant l(\mu)$, $k_{0}=k_{n+1}:=0$ and the $\beta_{r}$ are as in (1.11).

Note that both sides of (1.13) trivially vanish unless $l(\lambda) \leqslant k_{1}$ so that without loss of generality it may be assumed that $\lambda \in \mathscr{P}_{k_{1}}$. Then the $r=1$ term in the second double product on the right simplifies to 1 upon choosing $\ell=k_{1}$.

Since

$$
C_{\gamma}^{k_{1}}[0,1]=\left\{t \in \mathbb{R}^{k_{1}}: 0<t_{1}<t_{2}<\cdots<t_{k_{1}}<1\right\},
$$

Theorem 1.2 for $n=1$ is equivalent to Theorem 1.1. For $\mu=0$ the theorem corresponds to the $\mathrm{A}_{n}$ analogue of Kadell's integral [63, Theorem 6.1], and for $\beta=\gamma$ it gives an $\mathrm{A}_{n}$ analogue of the Hua-Kadell integral [19, 23. Our proof of Theorem 1.2 is not reliant on the Okounkov-Olshanski integral formula for Jack polynomials and instead uses $\mathrm{A}_{n}$ Cauchy-type identities for Macdonald polynomials. One advantage of our approach is that it immediately implies a companion to the $\mathrm{A}_{n}$ AFLT integral, stated as Theorem 4.1 in Section 4.

Setting $k_{1}=0$ in the $\mathrm{A}_{n}$ Selberg integral yields the $\mathrm{A}_{n-1}$ Selberg integral. The same is not true, however, for the $\mathrm{A}_{n}$ AFLT integral. Setting $k_{1}=0$ in (1.13) forces $\lambda=0$ for nonvanishing, thus eliminating one of the two Jack polynomials in the integrand. In their work on the AGT conjecture for $\mathrm{WA}_{n}$, Matsuo and Zhang [66] formulated several conjectures for $\mathrm{A}_{n}$ Selberg integrals of AFLT type that do have the desired reduction property, but unfortunately as stated their conjectures appear to be false. In the $\gamma \rightarrow 1$ limit of Theorem 1.2, in which case the Jack polynomials $P_{\lambda}^{(1 / \gamma)}\left[t^{(1)}\right]$ and $P_{\mu}^{(1 / \gamma)}\left[t^{(n)}+\beta / \gamma-1\right]$ simplify to the Schur functions $s_{\lambda}\left[t^{(1)}\right]$ and $s_{\mu}\left[t^{(n)}+\beta-1\right]$, 
we have managed to prove a corrected Matsuo-Zhang-type AFLT integral, containing a product of $n+1$ Schur functions in the integrand. Because (1.8a) implies that $|\operatorname{Re}(\gamma)|<1$, in this integral we must replace the real domain of integration $C_{\gamma}^{k_{1}, \ldots, k_{n}}[0,1]$ by the complex contour

$$
C^{k_{1}, \ldots, k_{n}}=C_{1}^{k_{1}} \times \cdots \times C_{n}^{k_{n}}, \quad \text { where } \quad C_{r}^{k_{r}}=\underbrace{C_{r} \times \cdots \times C_{r}}_{k_{r} \text { times }}
$$

defined as follows. Each $C_{r}$ is a positively oriented Jordan curve which passes through the origin, contains the interval $(0,1]$ in its interior, and has nonzero slope close to 0 . Away from 0 , the contour $C_{r}$ for $r \geqslant 2$ is contained in the interior of $C_{r-1}$ as shown in the figure below

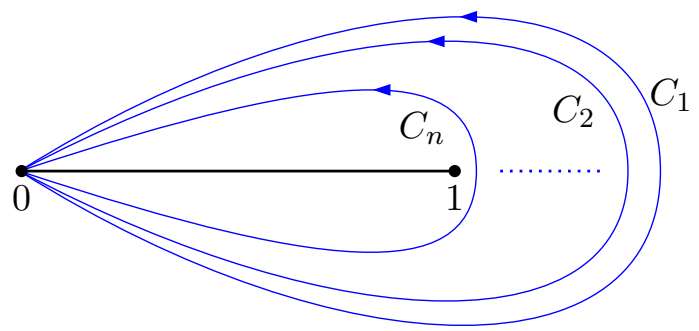

For $\gamma=1$ we now redefine the $\mathrm{A}_{n}$ Selberg average as follows. In the complex $t_{i}^{(r)}$-plane fix the usual principal branch of the complex logarithm, with cut along the negative real axis and argument in $(-\pi, \pi]$. Then for $0 \leqslant k_{1} \leqslant \cdots \leqslant k_{n}$ and $\alpha_{1}, \ldots, \alpha_{n}, \beta \in \mathbb{C}$ such that

$$
\operatorname{Re}\left(\alpha_{r}+\cdots+\alpha_{s}\right)>s-r \text { for } 1 \leqslant r \leqslant s \leqslant n
$$

we define

$$
\langle\mathscr{O}\rangle_{\alpha_{1}, \ldots, \alpha_{n}, \beta}^{k_{1}, \ldots, k_{n}}:=\frac{I_{k_{1}, \ldots, k_{n}}^{\mathrm{A}_{n}}\left(\mathscr{O} ; \alpha_{1}, \ldots, \alpha_{n}, \beta\right)}{I_{k_{1}, \ldots, k_{n}}^{\mathrm{A}_{n}}\left(1 ; \alpha_{1}, \ldots, \alpha_{n}, \beta\right)},
$$

where, assuming (1.11),

$$
\begin{aligned}
I_{k_{1}, \ldots, k_{n}}^{\mathrm{A}_{n}}\left(\mathscr{O} ; \alpha_{1}, \ldots, \alpha_{n}, \beta\right) & \\
:=\frac{1}{(2 \pi \mathrm{i})^{k_{1}+\cdots+k_{n}}} \int_{C^{k_{1}, \ldots, k_{n}}} \mathscr{O}\left(t^{(1)}, \ldots, t^{(n)}\right) & \prod_{r=1}^{n} \prod_{i=1}^{k_{r}}\left(t_{i}^{(r)}\right)^{\alpha_{r}-1}\left(t_{i}^{(r)}-1\right)^{\beta_{r}-1} \\
& \times \prod_{r=1}^{n} \Delta^{2}\left(t^{(r)}\right) \prod_{r=1}^{n-1} \Delta^{-1}\left(t^{(r)}, t^{(r+1)}\right) \mathrm{d} t^{(1)} \cdots \mathrm{d} t^{(n)} .
\end{aligned}
$$

The integral (1.17) should be understood in the sense of indefinite integrals since the integrand is not defined at $t_{i}^{(r)}=0 \in C_{r}$. Due to the change in contour, the normalisation is now given by (see (5.9) )

$$
\begin{aligned}
& I_{k_{1}, \ldots, k_{n}}^{\mathrm{A}_{n}}\left(1 ; \alpha_{1}, \ldots, \alpha_{n}, \beta\right) \\
& \quad=\prod_{r=1}^{n}\left((-1)\left(\begin{array}{c}
k_{r} \\
2
\end{array}\right) \prod_{i=1}^{k_{r}} \frac{i !}{\Gamma\left(k_{r+1}-\beta_{r}+2-i\right)}\right)
\end{aligned}
$$




$$
\times \prod_{1 \leqslant r \leqslant s \leqslant n} \prod_{i=1}^{k_{r}-k_{r-1}} \frac{\Gamma\left(\alpha_{r}+\cdots+\alpha_{s}+r-s+i-1\right)}{\Gamma\left(\alpha_{r}+\cdots+\alpha_{s}+\beta_{s}+k_{s}-k_{s+1}+r-s+i-2\right)},
$$

where $k_{0}=k_{n+1}:=0$.

Let $t^{(0)}:=0$. Then our next main result is a closed form evaluation of

$$
\left\langle\left(\prod_{r=1}^{n} s_{\lambda^{(r)}}\left[t^{(r)}-t^{(r-1)}\right]\right) s_{\lambda^{(n+1)}}\left[t^{(n)}+\beta-1\right]\right\rangle_{\alpha_{1}, \ldots, \alpha_{n}, \beta}^{k_{1}, \ldots, k_{n}},
$$

generalising the $\gamma=1$ case of (1.13). The most concise way to state this is by using the duality [32, p. 43]

$$
s_{\mu^{\prime}}[X]=(-1)^{|\mu|} s_{\mu}[-X],
$$

and to instead give the evaluation of

$$
\left\langle\prod_{r=1}^{n+1} s_{\lambda^{(r)}}\left[t^{(r)}-t^{(r-1)}\right]\right\rangle_{\alpha_{1}, \ldots, \alpha_{n}, \beta}^{k_{1}, \ldots, k_{n}},
$$

where $t^{(n+1)}:=1-\beta 3$ Before stating this evaluation we introduce the following shorthand notation. For $1 \leqslant r \leqslant n+1$ let

$$
A_{r}:=\alpha_{r}+\cdots+\alpha_{n}+k_{r}-k_{r-1}+r
$$

and $A_{r, s}:=A_{r}-A_{s}$, so that $A_{r, s}=-A_{s, r}$. In particular,

$$
A_{r, s}=\alpha_{r}+\cdots+\alpha_{s-1}+k_{r}-k_{r-1}-k_{s}+k_{s-1}+r-s
$$

for $1 \leqslant r \leqslant s \leqslant n+1$.

Theorem 1.3. For $n$ a positive integer, let $0 \leqslant k_{1} \leqslant \cdots \leqslant k_{n}$ be integers, $\alpha_{1}, \ldots, \alpha_{n}, \beta \in \mathbb{C}$ such that (1.16) holds, and $\lambda^{(1)}, \ldots, \lambda^{(n+1)} \in \mathscr{P}$. Let $t^{(0)}:=0$ and $t^{(n+1)}:=1-\beta$. Then

$$
\begin{aligned}
& \left\langle\prod_{r=1}^{n+1} s_{\lambda(r)}\left[t^{(r)}-t^{(r-1)}\right]\right\rangle_{\alpha_{1}, \ldots, \alpha_{n}, \beta}^{k_{1}, \ldots, k_{n}} \\
& =\prod_{r=1}^{n+1} \prod_{1 \leqslant i<j \leqslant \ell_{r}} \frac{\lambda_{i}^{(r)}-\lambda_{j}^{(r)}+j-i}{j-i} \prod_{r, s=1}^{n+1} \prod_{i=1}^{\ell_{r}} \frac{\left(A_{r, s}-k_{s-1}+k_{s}-i+1\right)_{\lambda_{i}(r)}}{\left(A_{r, s}+\ell_{s}-i+1\right)_{\lambda_{i}(r)}} \\
& \quad \times \quad \prod_{1 \leqslant r<s \leqslant n+1} \prod_{i=1}^{\ell_{r}} \prod_{j=1}^{\ell_{s}} \frac{\lambda_{i}^{(r)}-\lambda_{j}^{(s)}+A_{r, s}+j-i}{A_{r, s}+j-i},
\end{aligned}
$$

where $k_{0}:=0$ and $k_{n+1}:=1-\beta$, and where $\ell_{r}(1 \leqslant r \leqslant n+1)$ is an arbitrary nonnegative integer such that $\ell_{r} \geqslant l\left(\lambda^{(r)}\right)$.

The reader is warned that in order to obtain the above compact form for the right-hand side we have used a different convention for $k_{n+1}$ than in the previous two theorems. We also remark that

\footnotetext{
${ }^{3}$ For the evaluation of the average (1.19) see equation (7.1) below.
} 
(1.22) displays a significant amount of nontrivial cancellation. For $s=r$ the second triple product on the right becomes

$$
\prod_{r=1}^{n+1} \prod_{i=1}^{\ell_{r}} \frac{\left(k_{r}-k_{r-1}-i+1\right)_{\lambda_{i}^{(r)}}}{\left(\ell_{r}-i+1\right)_{\lambda_{i}^{(r)}}}
$$

Since $\ell_{r} \geqslant l\left(\lambda^{(r)}\right)$, this shows that the right-hand side vanishes unless $l\left(\lambda^{(r)}\right) \leqslant k_{r}-k_{r-1}$ for all $1 \leqslant r \leqslant n$. The integrand, however, only vanishes for $l\left(\lambda^{(1)}\right) \leqslant k_{1}-k_{0}=k_{1}$. Finally we note that (1.22) has the desired rank-reduction property. If we denote either side of (1.22) by

$$
I_{\lambda(1), \ldots, \lambda(n+1)}^{k_{1}, \ldots, k_{n}}\left(\alpha_{1}, \ldots, \alpha_{n}, \beta\right),
$$

then it is readily verified that

$$
I_{0, \lambda(2), \ldots, \lambda(n+1)}^{0, k_{2}, \ldots, k_{n}}\left(\alpha_{1}, \alpha_{2}, \ldots, \alpha_{n}, \beta\right)=I_{\lambda^{(2)}, \ldots, \lambda(n+1)}^{k_{2}, \ldots, k_{n}}\left(\alpha_{2}, \ldots, \alpha_{n}, \beta\right) .
$$

Evaluating the Selberg integral (1.4) for $\gamma=1$ is not at all hard; it follows from Heine's integral formula for the Hankel determinant of moments of orthogonal polynomials on the real line applied to the case of Jacobi polynomials, see e.g., [30, 47. We have not found a similarly elementary proof of Theorem 1.3, and our proof hinges on a novel type of integration formula for Schur functions indexed by sequences of complex numbers, see Theorem 5.3 .

Our third main result applies to $A_{1}$, and is an elliptic generalisation of the AFLT integral (1.6). This integral, which also generalises the elliptic Selberg integral of 9, 10, 44, contains two elliptic skew interpolation functions in the integrand. These play the role of the pair of Jack polynomials in the AFLT integral.

For $\boldsymbol{\lambda}, \boldsymbol{\nu}$ a pair of bipartitions, let $R_{\boldsymbol{\lambda} / \boldsymbol{\nu}}^{*}\left(\left[v_{1}, \ldots, v_{2 m}\right] ; a, b ; t, p, q\right)$ denote an elliptic skew interpolation function [45, for which we use the shorthand notation

$$
\begin{aligned}
R_{\boldsymbol{\lambda} / \nu}^{*}\left(\left[u z_{1}^{ \pm}, \ldots, u z_{n}^{ \pm}, v_{1}, \ldots, v_{2 m}\right]\right. & ; a, b ; t ; p, q) \\
& :=R_{\lambda / \nu}^{*}\left(\left[u z_{1}, u z_{1}^{-1}, \ldots, u z_{n}, u z_{n}^{-1}, v_{1}, \ldots, v_{2 m}\right] ; a, b ; t ; p, q\right) .
\end{aligned}
$$

Further let $\left(a_{1}, \ldots, a_{k} ; q\right)_{\infty}:=\prod_{r=1}^{k} \prod_{i=0}^{\infty}\left(1-a_{r} q^{i}\right)$ and let $\Gamma(z ; p, q)$ denote the elliptic gamma function [49, for which we adopt the usual multiplicative plus-minus conventions

$$
\begin{aligned}
\Gamma\left(z^{ \pm} ; p, q\right) & :=\Gamma(z ; p, q) \Gamma\left(z^{-1} ; p, q\right), \\
\Gamma\left(z^{ \pm} w^{ \pm} ; p, q\right) & :=\Gamma(z w ; p, q) \Gamma\left(z w^{-1} ; p, q\right) \Gamma\left(z^{-1} w ; p, q\right) \Gamma\left(z^{-1} w^{-1} ; p, q\right) .
\end{aligned}
$$

For $\boldsymbol{\lambda}=\left(\lambda^{(1)}, \lambda^{(2)}\right) \in \mathscr{P}_{n}^{2}$ we define the following shorthand for the ratio of products of elliptic gamma functions,

$$
\Delta_{\lambda}^{0}\left(a \mid b_{1}, \ldots, b_{k} ; t ; p, q\right):=\Delta_{\lambda^{(1)}}^{0}\left(a \mid b_{1}, \ldots, b_{k} ; q, t ; p\right) \Delta_{\lambda^{(2)}}^{0}\left(a \mid b_{1}, \ldots, b_{k} ; p, t ; q\right),
$$

with

$$
\Delta_{\lambda}^{0}\left(a \mid b_{1}, \ldots, b_{k} ; q, t ; p\right):=\prod_{r=1}^{k} \prod_{i=1}^{n} \frac{\Gamma\left(t^{1-i} q^{\lambda_{i}} b_{r} ; p, q\right) \Gamma\left(p q t^{1-i} a / b_{r} ; p, q\right)}{\Gamma\left(t^{1-i} b_{r} ; p, q\right) \Gamma\left(p q t^{1-i} q^{\lambda_{i}} a / b_{r} ; p, q\right)} .
$$

In particular, for $\mu \in \mathscr{P}_{m}$,

$$
\Delta_{\mu}^{0}\left(a \mid b\langle\boldsymbol{\lambda}\rangle_{n ; t ; p, q} ; q, t ; p\right)=\prod_{i=1}^{n} \prod_{j=1}^{m} \frac{\Gamma\left(t^{n-i-j+1} q^{\lambda_{i}^{(1)}+\mu_{j}} p^{\lambda_{i}^{(2)}} b ; p, q\right) \Gamma\left(p q t^{i-j-n+1} q^{-\lambda_{i}^{(1)}} p^{-\lambda_{i}^{(2)}} a / b ; p, q\right)}{\Gamma\left(t^{n-i-j+1} q^{\lambda_{i}^{(1)}} p^{\lambda_{i}^{(2)}} b ; p, q\right) \Gamma\left(p q t^{i-j-n+1} q^{-\lambda_{i}^{(1)}+\mu_{j}} p^{-\lambda_{i}^{(2)}} a / b ; p, q\right)},
$$


where

$$
\langle\boldsymbol{\lambda}\rangle_{n ; t ; p, q}:=\left(q^{\lambda_{1}^{(1)}} p^{\lambda_{1}^{(2)}} t^{n-1}, q^{\lambda_{2}^{(1)}} p^{\lambda_{2}^{(2)}} t^{n-2}, \ldots, q^{\lambda_{n-1}^{(1)}} p^{\lambda_{n-1}^{(2)}} t, q^{\lambda_{n}^{(1)}} p^{\lambda_{n}^{(2)}}\right),
$$

is the spectral vector for the bipartition $\boldsymbol{\lambda}$. With the above notation we may state the following elliptic analogue of (1.6), where we note that instead of $k$ we use $n$ to denote the number of integration variables.

Theorem 1.4 (Elliptic AFLT integral). Let $n$ be a positive integer, $p, q, t, t_{1}, t_{2}, t_{3}, t_{4}, t_{5}, t_{6} \in \mathbb{C}$ such that the elliptic balancing condition

$$
t^{2 n-2} t_{1} \cdots t_{6}=p q
$$

holds and such that $|p|,|q|<1$. Further let

$$
\kappa_{n}:=\frac{(p ; p)_{\infty}^{n}(q ; q)_{\infty}^{n} \Gamma^{n}(t ; p, q)}{2^{n} n !(2 \pi \mathrm{i})^{n}} .
$$

Then, for $\boldsymbol{\lambda} \in \mathscr{P}_{n}^{2}$ and $\boldsymbol{\mu} \in \mathscr{P}^{2}$,

$$
\begin{aligned}
\kappa_{n} \int_{C_{\boldsymbol{\lambda}}} & R_{\boldsymbol{\lambda} / \mathbf{0}}^{*}\left(\left[t^{1 / 2} z_{1}^{ \pm}, \ldots, t^{1 / 2} z_{n}^{ \pm}\right] ; t^{n-1 / 2} t_{1}, t^{1 / 2} t_{2} ; t ; p, q\right) \\
& \times R_{\boldsymbol{\mu} / \mathbf{0}}^{*}\left(\left[t^{1 / 2} z_{1}^{ \pm}, \ldots, t^{1 / 2} z_{n}^{ \pm}, t^{-1 / 2} t_{4}, t^{-1 / 2} t_{5}\right] ; t^{n-3 / 2} t_{3} t_{4} t_{5}, t^{1 / 2} t_{6} ; t ; p, q\right) \\
& \times \prod_{1 \leqslant i<j \leqslant n} \frac{\Gamma\left(t z_{i}^{ \pm} z_{j}^{ \pm} ; p, q\right)}{\Gamma\left(z_{i}^{ \pm} z_{j}^{ \pm} ; p, q\right)} \prod_{i=1}^{n} \frac{\prod_{r=1}^{6} \Gamma\left(t_{r} z_{i}^{ \pm} ; p, q\right)}{\Gamma\left(z_{i}^{ \pm 2} ; p, q\right)} \frac{\mathrm{d} z_{1}}{z_{1}} \cdots \frac{\mathrm{d} z_{n}}{z_{n}} \\
= & \prod_{i=1}^{n}\left(\Gamma\left(t^{i} ; p, q\right) \prod_{1 \leqslant r<s \leqslant 6} \Gamma\left(t^{i-1} t_{r} t_{s} ; p, q\right)\right) \\
& \times \Delta_{\boldsymbol{\lambda}}^{0}\left(t^{n-1} t_{1} / t_{2} \mid t^{n}, t^{n-1} t_{1} t_{3}, t^{n-1} t_{1} t_{4}, t^{n-1} t_{1} t_{5}, t^{n-1} t_{1} t_{6} ; t ; p, q\right) \\
& \times \Delta_{\boldsymbol{\mu}}^{0}\left(t^{n-2} t_{3} t_{4} t_{5} / t_{6} \mid t^{n-1} t_{3} t_{4}, t^{n-1} t_{3} t_{5}, t^{n-1} t_{4} t_{5} ; t ; p, q\right) \\
& \times \frac{\Delta_{\boldsymbol{\mu}}^{0}\left(t^{n-2} t_{3} t_{4} t_{5} / t_{6} \mid t^{n-2} t_{1} t_{3} t_{4} t_{5}\langle\boldsymbol{\lambda}\rangle_{n ; t ; p, q}\right)}{\Delta_{\boldsymbol{\mu}}^{0}\left(t^{n-2} t_{2} t_{3} t_{4} / t_{5} \mid t^{n-1} t_{1} t_{3} t_{4} t_{5}\langle\boldsymbol{\lambda}\rangle_{n ; t ; p, q}\right)},
\end{aligned}
$$

where $C_{\boldsymbol{\lambda} \boldsymbol{\mu}}$ is a deformation of $\mathbb{T}^{n}$ (with $\mathbb{T}$ the positively oriented unit circle) separating sequences of poles of the integrand tending to zero from sequences of poles tending to infinity.

For $\boldsymbol{\mu}=0$ this may be viewed as an analogue of Kadell's integral, and also follows by setting $\boldsymbol{\mu}=0$ in (6.11) below, a fact that was already noted by the second author in [4, Remark 2]. Imposing the constraint $t_{4} t_{5}=t$, the integral may be viewed as an elliptic analogue of the HuaKadell integral. In this case the integral is invariant under the simultaneous substitutions $\boldsymbol{\lambda} \leftrightarrow \boldsymbol{\mu}$ and $\left(t_{1}, t_{2}\right) \leftrightarrow\left(t_{3}, t_{6}\right)$.

By taking an appropriate $p \rightarrow 0$ limit, Theorem 1.4 simplifies to the following AFLT integral over a pair of Macdonald polynomials $P_{\lambda}(q, t)$.

Corollary 1.5. For $\lambda \in \mathscr{P}_{n}, \mu \in \mathscr{P}$, and $a, b, q, t \in \mathbb{C}$ such that $|b|,|q|,|t|<1$,

$$
\frac{1}{n !(2 \pi \mathrm{i})^{n}} \int_{\mathbb{T}^{n}} P_{\lambda}(z ; q, t) P_{\mu}\left(\left[z+\frac{t-b}{1-t}\right] ; q, t\right)
$$




$$
\begin{gathered}
\times \prod_{i=1}^{n} \frac{\left(a / z_{i}, q z_{i} / a ; q\right)_{\infty}}{\left(b / z_{i}, z_{i} ; q\right)_{\infty}} \prod_{1 \leqslant i<j \leqslant n} \frac{\left(z_{i} / z_{j}, z_{j} / z_{i} ; q\right)_{\infty}}{\left(t z_{i} / z_{j}, t z_{j} / z_{i} ; q\right)_{\infty}} \frac{\mathrm{d} z_{1}}{z_{1}} \cdots \frac{\mathrm{d} z_{n}}{z_{n}} \\
=b^{|\lambda|} t^{|\mu|} P_{\lambda}\left(\left[\frac{1-t^{n}}{1-t}\right] ; q, t\right) P_{\mu}\left(\left[\frac{1-b t^{n-1}}{1-t}\right] ; q, t\right) \\
\times \prod_{i=1}^{n} \frac{\left(t, a t^{n-m-i} q^{\lambda_{i}}, a t^{1-i} / b, q t^{i-1} b / a ; q\right)_{\infty}}{\left(q, t^{i}, b t^{i-1}, a t^{1-i} q^{\lambda_{i}} / b ; q\right)_{\infty}} \prod_{i=1}^{m} \prod_{j=1}^{m} \frac{\left(a t^{n-i-j+1} q^{\lambda_{i}+\mu_{j}} ; q\right)_{\infty}}{\left(a t^{n-i-j} q^{\lambda_{i}+\mu_{j}} ; q\right)_{\infty}}
\end{gathered}
$$

where $m$ is an arbitrary integer such that $m \geqslant l(\mu)$.

For $\lambda=\mu=0$ the above integral may also be found in [6, Section 6] as a special case of the biorthogonality relation for multivariable Pastro polynomials. This special case also easily follows by combining the ${ }_{1} \Psi_{1}$ summation for Macdonald polynomials [27] with the orthogonality and quadratic norm evaluation of the Macdonald polynomials with respect to the scalar product $\langle\cdot, \cdot\rangle_{n}^{\prime}$, see (6.13).

The remainder of this paper is organised as follows. In the next section we review some basic material from the theory of symmetric functions. This includes a discussion of Schur, Jack and Macdonald polynomials as well as the heavily-used plethystic notation. In Section 3 we present some $\mathrm{A}_{n}$ generalisations of the classical Cauchy identity for Macdonald polynomials. Then, in Section 4, we show that such Cauchy identities are essentially discrete analogues of $\mathrm{A}_{n}$ AFLT integrals, leading to a proof of Theorem 1.2 and its companion given in Theorem 4.1. In Section 5 we use integral formulas of Cauchy-type for complex Schur functions to prove Theorem 1.3. In Section [6 we review some of the theory of elliptic interpolation functions and use this to prove the elliptic AFLT integral of Theorem 1.4. Finally, in Section 7, we discuss some open problems stemming from our work.

\section{Preliminaries}

2.1. Partitions. Throughout this paper $\mathbb{N}$ denotes the set of nonnegative integers.

A partition $\lambda$ is a weakly decreasing sequence of nonnegative integers $\left(\lambda_{1}, \lambda_{2}, \ldots\right)$ with only finitely many of the $\lambda_{i}$ nonzero. The positive $\lambda_{i}$ are called the parts of $\lambda$, and the number of parts is called the length, denoted by $l(\lambda)$. The sum of the $\lambda_{i}$ is denoted by $|\lambda|$, and if $|\lambda|=n$ we say that $\lambda$ is a partition of $n$ and write $\lambda \vdash n$. With the possible exception of finitely many zeros, we usually drop the infinite string of zeros of a partition so that $\lambda=\left(\lambda_{1}, \ldots, \lambda_{n}\right)$ denotes a partition of length at most $n$. The set of all partitions and the set of partitions of length at most $n$ are denoted by $\mathscr{P}$ and $\mathscr{P}_{n}$ respectively. In particular, $\mathscr{P}_{0}=\{0\}$ where, by mild abuse of notation, the unique partition of zero is denoted by 0 .

We identify a partition with its Young diagram, which is the set of all $(i, j) \in \mathbb{N}^{2}$ such that $1 \leqslant i \leqslant l(\lambda)$ and $1 \leqslant j \leqslant \lambda_{i}$. This may be visualised as a left-justified array of squares with $\lambda_{i}$ squares in row $i$. For example the Young diagram of the partition $(6,4,3,1,1)$ is

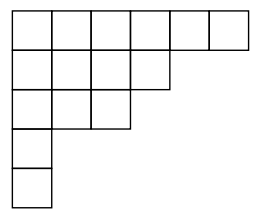


The conjugate of $\lambda$, which is denoted by $\lambda^{\prime}$, is obtained by reflecting $\lambda$ in the main diagonal. Hence $(6,4,3,1,1)^{\prime}=(5,3,3,2,1,1)$. Given a square $s=(i, j) \in \mathbb{N}^{2}$ and a partition $\lambda$ we define the (generalised) arm-length, leg-length, arm-colength, and leg-colength of $s$ by

$$
\begin{aligned}
a_{\lambda}(s) & :=\lambda_{i}-j, & l_{\lambda}(s) & :=\lambda_{j}^{\prime}-i, \\
a^{\prime}(s) & :=j-1, & l^{\prime}(s) & :=i-1,
\end{aligned}
$$

respectively. If $s \in \lambda$ this reduces to the standard definition in [32, p. 337]. Note that with the above notation the function $E$ in (1.3) may also be written as

$$
E(u, \lambda, \mu, s)=u-b l_{\mu}(s)+b^{-1}\left(a_{\lambda}(s)+1\right),
$$

where $s \in \lambda$.

A frequently encountered statistic on partitions is

$$
n(\lambda):=\sum_{i \geqslant 1}(i-1) \lambda_{i}=\sum_{i \geqslant 1}\left(\begin{array}{c}
\lambda_{i}^{\prime} \\
2
\end{array}\right)=\sum_{s \in \lambda} l^{\prime}(s) .
$$

All of the previous notation regarding partitions is extended to bipartitions $\lambda \in \mathscr{P}^{2}$ in the obvious way. In particular, $\boldsymbol{\mu} \subseteq \boldsymbol{\lambda}$ will be used as shorthand for $\mu^{(1)} \subseteq \lambda^{(1)}$ and $\mu^{(2)} \subseteq \lambda^{(2)}$, and $\mathbf{0}:=(0,0)$.

2.2. Generalised shifted factorials. For $n$ a nonnegative integer and $b$ an indeterminate or complex number, the Pochhammer symbol $(b)_{n}$ is defined as

$$
(b)_{n}:=\prod_{i=0}^{n-1}(b+i)
$$

where an empty product is to be taken as 1 . We generalise this to complex $z$ by

$$
(b)_{z}:=\frac{\Gamma(b+z)}{\Gamma(b)}
$$

where now it is assumed that $b \in \mathbb{C}$ and neither $b$ nor $b+z$ are nonpositive integers. Similarly, for indeterminate or arbitrary complex $b$ and $q$, the $q$-shifted factorial is defined as

$$
(b ; q)_{n}:=\prod_{i=0}^{n-1}\left(1-b q^{i}\right)
$$

where $n \in \mathbb{N} \cup\{\infty\}$ and where $|q|<1$ in the infinite product case. When $0<q<1$ this can again be extended to complex $z$ by

$$
(b ; q)_{z}:=\frac{(b ; q)_{\infty}}{\left(b q^{z} ; q\right)_{\infty}}
$$

This in particular implies that for $n$ a positive integer,

$$
\frac{1}{(q ; q)_{-n}}=0
$$

In terms of the $q$-gamma function

$$
\Gamma_{q}(z):=\frac{(q ; q)_{\infty}}{\left(q^{z} ; q\right)_{\infty}}(1-q)^{1-z}
$$


and (for $z \in \mathbb{C} \backslash\{0,-1,-2, \ldots\}$ and $0<q<1$ ),

$$
\left(q^{b} ; q\right)_{n}=(1-q)^{n} \frac{\Gamma_{q}(b+n)}{\Gamma_{q}(b)} .
$$

A generalisation of (2.3) to partitions is given by

$$
(b ; q, t)_{\lambda}:=\prod_{s \in \lambda}\left(1-b q^{a^{\prime}(s)} t^{-l^{\prime}(s)}\right)=\prod_{i \geqslant 1}\left(b t^{1-i} ; q\right)_{\lambda_{i}} .
$$

Setting $t=q^{\gamma}$ and replacing $b$ by $q^{b}$, and then letting $q$ tend to 1 we obtain an analogue of the Pochhammer symbol indexed by partitions

$$
(b ; \gamma)_{\lambda}:=\prod_{i \geqslant 1}(b+(1-i) \gamma)_{\lambda_{i}} .
$$

Also frequently used in this paper are the generalised hook polynomials

$$
\begin{aligned}
& c_{\lambda}(q, t):=\prod_{s \in \lambda}\left(1-q^{a_{\lambda}(s)} t^{l_{\lambda}(s)+1}\right)=\prod_{i=1}^{n}\left(t^{n-i+1} ; q\right)_{\lambda_{i}} \prod_{1 \leqslant i<j \leqslant n} \frac{\left(t^{j-i} ; q\right)_{\lambda_{i}-\lambda_{j}}}{\left(t^{j-i+1} ; q\right)_{\lambda_{i}-\lambda_{j}}} \\
& c_{\lambda}^{\prime}(q, t):=\prod_{s \in \lambda}\left(1-q^{a_{\lambda}(s)+1} t^{l_{\lambda}(s)}\right)=\prod_{i=1}^{n}\left(q t^{n-i} ; q\right)_{\lambda_{i}} \prod_{1 \leqslant i<j \leqslant n} \frac{\left(q t^{j-i-1} ; q\right)_{\lambda_{i}-\lambda_{j}}}{\left(q t^{j-i} ; q\right)_{\lambda_{i}-\lambda_{j}}} \\
& b_{\lambda}(q, t):=\frac{c_{\lambda}(q, t)}{c_{\lambda}^{\prime}(q, t)}
\end{aligned}
$$

Note that the choice of $n$ on the right of the first two equations is irrelevant as long as $n \geqslant l(\lambda)$.

Finally, at the top-level we have the elliptic shifted factorials and gamma function. To define the former we need the modified theta function

$$
\theta(z ; p):=(z ; p)_{\infty}(p / z ; p)_{\infty},
$$

where $z \in \mathbb{C}^{*}$ and $p \in \mathbb{C}$ such that $|p|<1$. Then the elliptic shifted factorial is given by

$$
(b ; q, p)_{n}:=\prod_{i=0}^{n-1} \theta\left(b q^{i} ; p\right),
$$

so that $(b ; q, 0)_{n}=(b ; q)_{n}$. If $\Gamma(z ; p, q)$ denotes the elliptic gamma function [49]

$$
\Gamma(z ; p, q):=\prod_{i, j=0}^{\infty} \frac{1-p^{i+1} q^{j+1} / z}{1-z p^{i} q^{j}},
$$

which satisfies the reflection formula $\Gamma(z ; p, q) \Gamma(p q / z ; p, q)=1$, then, in analogy with (2.2),

$$
(b ; q, p)_{n}=\frac{\Gamma\left(b q^{n} ; p, q\right)}{\Gamma(b ; p, q)} .
$$

Similarly, the elliptic generalisation of (2.5) is

$$
(b ; q, t ; p)_{\lambda}:=\prod_{s \in \lambda} \theta\left(b q^{a^{\prime}(s)} t^{-l^{\prime}(s)} ; p\right)=\prod_{i \geqslant 1}\left(b t^{1-i} ; q, p\right)_{\lambda_{i}}
$$


To shorten some of our expressions we also use the following shorthand for the "well-poised" ratio of products of elliptic shifted factorials:

$$
\Delta_{\lambda}^{0}\left(a \mid b_{1}, \ldots, b_{n} ; q, t ; p\right):=\prod_{i=1}^{n} \frac{\left(b_{i} ; q, t ; p\right)_{\lambda}}{\left(p q a / b_{i} ; q, t ; p\right)_{\lambda}},
$$

from which it is clear that which it is clear that

$$
\Delta_{\lambda}^{0}\left(a \mid b_{1}, \ldots, b_{n} ; q, t ; p\right)=\frac{1}{\Delta_{\lambda}^{0}\left(a \mid p q a / b_{1}, \ldots, p q a / b_{n} ; q, t ; p\right)} .
$$

Finally we need

$$
\begin{aligned}
& C_{\lambda}^{-}(b ; q, t ; p):=\prod_{s \in \lambda} \theta\left(b q^{a_{\lambda}(s)} t^{l_{\lambda}(s)} ; p\right) \\
& C_{\lambda}^{+}(b ; q, t ; p):=\prod_{(i, j) \in \lambda} \theta\left(b q^{\lambda_{i}+j-1} t^{2-\lambda_{j}^{\prime}-i} ; p\right),
\end{aligned}
$$

so that $c_{\lambda}(q, t)=C_{\lambda}^{-}(t ; q, t ; 0)$ and $c_{\lambda}^{\prime}(q, t)=C_{\lambda}^{-}(q ; q, t ; 0)$.

2.3. Symmetric functions. Let $X=\left\{x_{1}, x_{2}, \ldots\right\}$ be an alphabet of countably many variables and $X_{n}=\left\{x_{1}, \ldots, x_{n}\right\}$ an alphabet of cardinality $n$. Then we denote the ring of symmetric functions in $X$ (resp. $X_{n}$ ) over the field $\mathbb{F}$ by $\Lambda$ (resp. $\Lambda_{n}$ ), see 32]. Typically, we will work with $\mathbb{F}=\mathbb{Q}$, or the extensions $\mathbb{Q}(\gamma)$ and $\mathbb{Q}(q, t)$.

Given a sequence $\alpha=\left(\alpha_{1}, \alpha_{2}, \ldots\right)$ of nonnegative integers such that $|\alpha|:=\alpha_{1}+\alpha_{2}+\cdots$ is finite, we write $\alpha^{+}$for the unique partition obtained by reordering the $\alpha_{i}$. Then the monomial symmetric function indexed by the partition $\lambda$ is defined as

$$
m_{\lambda}(X):=\sum_{\alpha^{+}=\lambda} X^{\alpha}
$$

where $X^{\alpha}:=x_{1}^{\alpha_{1}} x_{2}^{\alpha_{2}} \cdots$. Further defining $m_{\lambda}\left(X_{n}\right):=\left.m_{\lambda}(X)\right|_{x_{i}=0}$ for $i>n$ it follows that $m_{\lambda}\left(X_{n}\right)=0$ if $l(\lambda)>n$. The sets $\left\{m_{\lambda}(X)\right\}$ and $\left\{m_{\lambda}\left(X_{n}\right)\right\}_{l(\lambda) \leqslant n}$ form bases of $\Lambda$ and $\Lambda_{n}$ respectively.

For $k$ a nonnegative integer the $k$ th complete and elementary symmetric functions are defined by

$$
h_{k}(X):=\sum_{1 \leqslant i_{1} \leqslant \cdots \leqslant i_{k}} x_{i_{1}} \cdots x_{i_{k}}
$$

and

$$
e_{k}(X):=\sum_{1 \leqslant i_{1}<\cdots<i_{k}} x_{i_{1}} \cdots x_{i_{k}}
$$

respectively. For $k$ a positive integer we set $e_{-k}=h_{-k}=0$. The generating functions for the complete symmetric functions is given by

$$
\sigma_{z}(X):=\sum_{k \geqslant 0} z^{k} h_{k}(X)=\prod_{i \geqslant 1} \frac{1}{1-z x_{i}}
$$

and

$$
\sum_{k \geqslant 0} z^{k} e_{k}(X)=\prod_{i \geqslant 1}\left(1+z x_{i}\right)=\frac{1}{\sigma_{-z}(X)} .
$$


We also require Newton power sums

$$
p_{k}(X):=\sum_{i \geqslant 1} x_{i}^{k}
$$

for $k$ a positive integer. These admit the generating function

$$
\psi_{z}(X):=\sum_{k \geqslant 1} \frac{z^{k} p_{k}(X)}{k}=-\sum_{i \geqslant 1} \log \left(1-z x_{i}\right),
$$

so that

$$
\sigma_{z}(X)=\mathrm{e}^{\psi_{z}(X)} .
$$

The most important family of classical symmetric functions are the Schur functions, defined by the usual ratio of alternants

$$
s_{\lambda}\left(X_{n}\right)=\frac{\operatorname{det}_{1 \leqslant i, j \leqslant n}\left(x_{i}^{\lambda_{j}+n-j}\right)}{\Delta\left(X_{n}\right)},
$$

for $l(\lambda) \leqslant n$ and 0 otherwise. The Schur functions indexed by partitions of length at most $n$ form a basis of $\Lambda_{n}$. From (2.10) it is not hard to derive the specialisation formula [32, p. 44]

$$
s_{\lambda}(\underbrace{1, \ldots, 1}_{n \text { times }})=\prod_{i=1}^{k} \frac{(n-i+1)_{\lambda_{i}}}{(k-i+1)_{\lambda_{i}}} \prod_{1 \leqslant i<j \leqslant k} \frac{\lambda_{i}-\lambda_{j}+j-i}{j-i},
$$

where $k$ is an arbitrary integer such that $k \geqslant l(\lambda)$.

2.4. Plethystic notation. We extensively use plethystic or $\lambda$-ring notation when dealing with symmetric functions, see e.g., [18, 29, 46]. For $X=\left\{x_{1}, x_{2}, \ldots\right\}$ an alphabet and $f(X)$ a symmetric function in $X$ we use the additive notation

$$
f\left(x_{1}, x_{2}, \ldots\right)=f(X)=f[X]=f\left[x_{1}+x_{2}+\cdots\right] .
$$

Hence $X+Y$, the sum of the alphabets $X$ and $Y$, is the disjoint union of these sets. The above notation forces

$$
p_{k}[X+Y]=p_{k}[X]+p_{k}[Y]
$$

A symmetric function acting on the difference of two alphabets is then defined as

$$
p_{k}[X-Y]:=p_{k}[X]-p_{k}[Y] \text {. }
$$

Observe that

$$
p_{k}[(X+Y)-Y]=p_{k}[X]
$$

as it should. Inside plethystic brackets we denote the empty alphabet by 0 . By (2.9) it follows that

$$
\sigma_{z}[X+Y]=\sigma_{z}[X] \sigma_{z}[Y] \quad \text { and } \quad \sigma_{z}[X-Y]=\frac{\sigma_{z}[X]}{\sigma_{z}[Y]}
$$

and hence $\sigma_{z}[-X] \sigma_{z}[X]=1$. Together with (2.8) this implies

$$
h_{k}[-X]=(-1)^{k} e_{k}[X]
$$

which, by the dual Jacobi-Trudi identity [32, p. 41], extends to Schur functions as (1.20).

For the Cartesian product of two alphabets we have

$$
p_{k}[X Y]=p_{k}[X] p_{k}[Y],
$$


which in particular implies that if $X=x$ is an alphabet containing a single letter then

$$
p_{k}[x Y]=x^{k} p_{k}[Y] \text {. }
$$

However, from (2.12) we also have

$$
p_{k}[n X]:=p_{k}[\underbrace{X+\cdots+X}_{n \text { times }}]=n p_{k}[X],
$$

so that

$$
f[n]=f[\underbrace{1+\cdots+1}_{n \text { times }}]=f(\underbrace{1, \ldots, 1}_{n \text { times }}) .
$$

We extend the above to any $z \in \mathbb{F}$ via

$$
p_{k}[z X]:=z p_{k}[X] .
$$

For $X=1$ we more simply write the above left-hand side as $p_{k}[z]$. A Note that this leads to some notational ambiguities, and whenever not clear from the context we will indicate if a symbol such as $x$ or $z$ represents a letter or a binomial element.

Finally, since the Cartesian product of the alphabets $\left\{1, t, t^{2}, \ldots\right\}=1+t+t^{2}+\cdots$ and $1-t$ is 1 , we adopt the standard convention of writing the former as $1 /(1-t)$. An often occurring composite alphabet is $(a-b) /(1-t)$ for which

$$
p_{k}\left[\frac{a-b}{1-t}\right]=\frac{a^{k}-b^{k}}{1-t^{k}}
$$

and

$$
\sigma_{1}\left[\frac{a-b}{1-t}\right]=\frac{(b ; t)_{\infty}}{(a ; t)_{\infty}}
$$

2.5. Macdonald polynomials. In this section we work with the ring of symmetric functions $\Lambda$ over $\mathbb{F}=\mathbb{Q}(q, t)$.

Let $\langle\cdot, \cdot\rangle: \Lambda \times \Lambda \rightarrow \mathbb{F}$ be the $q, t$-Hall scalar product on $\Lambda$ given by [32, p. 306]

$$
\left\langle p_{\lambda}, p_{\mu}\right\rangle:=\delta_{\lambda, \mu} z_{\lambda} \prod_{i \geqslant 1} \frac{1-q^{\lambda_{i}}}{1-t^{\lambda_{i}}},
$$

where $z_{\lambda}:=\prod_{i \geqslant 1} i^{m_{i}} m_{i} !$. The Macdonald polynomials $P_{\lambda}=P_{\lambda}(q, t)=P_{\lambda}(X ; q, t)$ are the unique symmetric functions such that

$$
\left\langle P_{\lambda}, P_{\mu}\right\rangle=0 \quad \text { if } \quad \lambda \neq \mu
$$

and

$$
P_{\lambda}=m_{\lambda}+\sum_{\mu<\lambda} u_{\lambda \mu} m_{\mu}, \quad u_{\lambda \mu} \in \mathbb{F}
$$

with respect to the usual dominance order on partitions. Like the Schur functions, $\left\{P_{\lambda}(X)\right\}$ and $\left\{P_{\lambda}\left(X_{n}\right)\right\}_{l(\lambda) \leqslant n}$ are bases for $\Lambda$ and $\Lambda_{n}$ respectively, and $P_{\lambda}\left(X_{n} ; q, t\right)=0$ if $l(\lambda)>n$.

We also require the skew Macdonald polynomials defined by

$$
P_{\lambda}([X+Y] ; q, t)=\sum_{\mu} P_{\lambda / \mu}(X ; q, t) P_{\mu}(Y ; q, t)
$$

\footnotetext{
${ }^{4}$ In [29, p. 32] Lascoux refers to a single letter alphabet $x$ as a rank-1 element of a $\lambda$-ring and $z \in \mathbb{F}$ as a binomial element, since for the latter $e_{k}[z]=\left(\begin{array}{c}z \\ k\end{array}\right)$ and $h_{k}[z]=\left(\begin{array}{c}z+k-1 \\ k\end{array}\right)$.
} 
where it should be noted that $P_{\lambda / 0}=P_{\lambda}$. We also note that $P_{\lambda / \mu}$ is homogeneous of degree $|\lambda / \mu|$ and vanishes unless $\mu \subseteq \lambda$ (cf. [32, p. 344]).

For $q=t$ the Macdonald polynomials reduce to the Schur functions (2.10). More generally, if we set $t=q^{\gamma}$ and let $q$ tend to 1 we obtain the Jack polynomials [55]

$$
P_{\lambda}^{(1 / \gamma)}(X)=\lim _{q \rightarrow 1} P_{\lambda}\left(X ; q, q^{\gamma}\right)
$$

Setting $Q_{\lambda / \mu}(q, t):=b_{\lambda}(q, t) P_{\lambda / \mu}(q, t) / b_{\mu}(q, t)$, the Macdonald polynomials satisfy the (skew) Cauchy identity [32, p. 345]

$$
\begin{aligned}
\sum_{\lambda} P_{\lambda}(X ; q, t) Q_{\lambda / \mu}(Y ; q, t) & =P_{\mu}(X ; q, t) \prod_{i, j \geqslant 1} \frac{\left(t x_{i} y_{j} ; q\right)_{\infty}}{\left(x_{i} y_{j} ; q\right)_{\infty}} \\
& =P_{\mu}(X ; q, t) \sigma_{1}\left[\frac{1-t}{1-q} X Y\right]
\end{aligned}
$$

where the second equality follows from (2.13) and (2.14).

Let

$$
{ }_{2} \phi_{1}\left[\begin{array}{c}
a, b \\
c
\end{array} ; q, z\right]:=\sum_{k=0}^{\infty} \frac{(a ; q)_{k}(b ; q)_{k}}{(c ; q)_{k}(q ; q)_{k}} z^{k}
$$

denote the usual $q$-analogue of the ${ }_{2} F_{1}$ Gauss hypergeometric function [17].

Lemma 2.1. Let $x$ and $y$ be single-letter alphabets. Then

$$
P_{(r)}([x-y] ; q, t)=x_{2}^{r} \phi_{1}\left[\begin{array}{l}
t^{-1}, q^{-r} \\
q^{1-r} t^{-1}
\end{array} ; q, \frac{y q}{x}\right] .
$$

Proof. If we set $\mu=0$ in (2.16) and then replace $(X, Y) \mapsto(x-y, 1)$ this yields

$$
\sum_{r \geqslant 0} \frac{(t ; q)_{r}}{(t ; t)_{r}} P_{(r)}([x-y] ; q, t)=\sigma_{1}\left[\frac{1-t}{1-q}(x-y)\right]=\frac{(t x ; q)_{\infty}(y ; q)_{\infty}}{(x ; q)_{\infty}(t y ; q)_{\infty}} .
$$

Using the $q$-binomial theorem [17, Equation (II.3)] to expand the right-hand side as a power series in $x$ and $y$ leads to

$$
\sum_{r \geqslant 0} \frac{(t ; q)_{r}}{(t ; t)_{r}} P_{(r)}([x-y] ; q, t)=\sum_{k, \ell \geqslant 0} \frac{(t ; q)_{\ell}\left(t^{-1} ; q\right)_{k}}{(q ; q)_{\ell}(q ; q)_{k}} x^{\ell}(t y)^{k} .
$$

Equating terms of homogeneous degree $r$ in $x, y$ gives

$$
\frac{(t ; q)_{r}}{(t ; t)_{r}} P_{(r)}([x-y] ; q, t)=\sum_{k=0}^{r} \frac{(t ; q)_{r-k}\left(t^{-1} ; q\right)_{k}}{(q ; q)_{r-k}(q ; q)_{k}} x^{r-k}(t y)^{k}
$$

which is equivalent to (2.17).

For $\lambda \in \mathscr{P}_{n}$ define the spectral vector

$$
\langle\lambda\rangle_{n}=\langle\lambda\rangle_{n ; q, t}:=\left(q^{\lambda_{1}} t^{n-1}, q^{\lambda_{2}} t^{n-2}, \ldots, q^{\lambda_{n-1}} t, q^{\lambda_{n}} t^{0}\right)
$$

which, depending on the context, we will also interpret plethystically as

$$
\langle\lambda\rangle_{n}=q^{\lambda_{1}} t^{n-1}+q^{\lambda_{2}} t^{n-2}+\cdots+q^{\lambda_{n-1}} t+q^{\lambda_{n}} t^{0} .
$$


Then the principal specialisation formula for Macdonald polynomials [32, p. 337] can be written as

$$
P_{\lambda}\left[\langle 0\rangle_{n}\right]=P_{\lambda}\left[\frac{1-t^{n}}{1-t}\right]=\frac{t^{n(\lambda)}\left(t^{n} ; q, t\right)_{\lambda}}{c_{\lambda}(q, t)},
$$

where from hereon we mostly suppress the dependence of the Macdonald polynomials on $q$ and $t$. By a polynomial argument (2.18) is equivalent to [32, p. 338]

$$
P_{\lambda}\left[\frac{1-a}{1-t}\right]=\frac{t^{n(\lambda)}(a ; q, t)_{\lambda}}{c_{\lambda}(q, t)}
$$

Replacing $(a, t) \mapsto\left(q^{z \gamma}, q^{\gamma}\right)$ and letting $q$ tend to one in (2.19) yields the following expression for the Jack polynomial evaluated at a binomial element $z$ :

$$
P_{\lambda}^{(1 / \gamma)}[z]=\frac{(z \gamma ; \gamma)_{\lambda}}{(k \gamma ; \gamma)_{\lambda}} \prod_{1 \leqslant i<j \leqslant k} \frac{((j-i+1) \gamma)_{\lambda_{i}-\lambda_{j}}}{((j-i) \gamma)_{\lambda_{i}-\lambda_{j}}}
$$

where $k$ is an arbitrary integer such that $k \geqslant l(\lambda)$. For $\gamma=1$ and $z=n$, with $n$ a nonnegative integer this reduces to (2.11).

The evaluation symmetry of the Macdonald polynomials [32, p. 332] may expressed in terms of spectral vectors as

$$
P_{\mu}\left[\langle 0\rangle_{n}\right] P_{\lambda}\left[\langle\mu\rangle_{n}\right]=P_{\lambda}\left[\langle 0\rangle_{n}\right] P_{\mu}\left[\langle\lambda\rangle_{n}\right]
$$

where $\lambda, \mu \in \mathscr{P}_{n}$. We require a more general form of this symmetry, which is a nonsymmetric version of [64, Proposition 2.1].

Lemma 2.2. For $\lambda \in \mathscr{P}_{n}$ and $\mu \in \mathscr{P}_{m}$,

$$
P_{\mu}\left[\frac{1-a}{1-t}\right] P_{\lambda}\left[a t^{-m}\langle\mu\rangle_{m}+\frac{1-a t^{-m}}{1-t}\right]=P_{\lambda}\left[\frac{1-a}{1-t}\right] P_{\mu}\left[a t^{-n}\langle\lambda\rangle_{n}+\frac{1-a t^{-n}}{1-t}\right] .
$$

Proof. For $m=n$ and $a \mapsto a t^{n}$ this is [64, Proposition 2.1]

$$
P_{\mu}\left[\frac{1-a t^{n}}{1-t}\right] P_{\lambda}\left[a\langle\mu\rangle_{n}+\frac{1-a}{1-t}\right]=P_{\lambda}\left[\frac{1-a t^{n}}{1-t}\right] P_{\mu}\left[a\langle\lambda\rangle_{n}+\frac{1-a}{1-t}\right] .
$$

Fixing an integer $m$ such that $l(\mu) \leqslant m \leqslant n$ we have

$$
a\langle\mu\rangle_{n}=a t^{n-m}\langle\mu\rangle_{m}+\frac{a-a t^{n-m}}{1-t} .
$$

Therefore (2.23) becomes

$$
P_{\mu}\left[\frac{1-a t^{n}}{1-t}\right] P_{\lambda}\left[a t^{n-m}\langle\mu\rangle_{m}+\frac{1-a t^{n-m}}{1-t}\right]=P_{\lambda}\left[\frac{1-a t^{n}}{1-t}\right] P_{\mu}\left[a\langle\lambda\rangle_{n}+\frac{1-a}{1-t}\right] .
$$

Scaling $a \mapsto a t^{-n}$ results in (2.22). Since this is symmetric in $m$ and $n$ the restriction $m \leqslant n$ may be dropped. 


\section{Cauchy-type identities}

An important special case of the Cauchy identity (2.16) - obtained by taking $\mu=0$, making the plethystic substitution $Y \mapsto(1-a) /(1-t)$ and using the specialisation formula (2.19) - is the Kaneko-Macdonald $q$-binomial theorem [26, 33]

$$
\sum_{\lambda} \frac{t^{n(\lambda)}(a ; q, t)_{\lambda}}{c_{\lambda}^{\prime}(q, t)} P_{\lambda}(X ; q, t)=\prod_{i \geqslant 1} \frac{\left(a x_{i} ; q\right)_{\infty}}{\left(x_{i} ; q\right)_{\infty}} .
$$

An $\mathrm{A}_{n}$ analogue of this formula is given in [63, Theorem 3.2] and then applied to prove the $\nu=0$ case of Theorem 1.2. In order to prove Theorem 1.2 in full we require an $\mathrm{A}_{n}$ Cauchy-type identity which simultaneously generalises (2.16) and [63, Theorem 3.2]. This is the content of Theorem 3.4 below.

3.1. Identities for skew Macdonald polynomials. For partitions $\lambda, \mu$ and $k \in \mathbb{N}, \ell \in \mathbb{N} \cup\{\infty\}$ such that $k \geqslant l(\lambda)$ and $\ell \geqslant l(\mu)$, define 64$]^{5}$

$$
f_{\lambda, \mu}^{k, \ell}(a ; q, t):=t^{-k|\mu|} \prod_{i=1}^{k} \prod_{j=1}^{\ell} \frac{\left(a q t^{j-i-1} ; q\right)_{\lambda_{i}-\mu_{j}}}{\left(a q t^{j-i} ; q\right)_{\lambda_{i}-\mu_{j}}} .
$$

By $(a ; q)_{-n} /(b ; q)_{-n}=(b / a)^{n}(q / b ; q)_{n} /(q / a ; q)_{n}$ it follows that

$$
f_{\lambda, \mu}^{k, \ell}(a ; q, t)=f_{\mu, \lambda}^{\ell, k}(t / a q ; q, t)
$$

provided $\ell$ is finite. For infinite $\ell$, let $t^{\ell}:=0$. Our proof of Theorem 3.4, given in Section 3.3, hinges on the following summation formula for skew Macdonald polynomials [64, Theorem 3.4].

Proposition 3.1. For partitions $\lambda$ and $\mu$, we have

$$
\sum_{\nu} t^{-|\nu|} P_{\mu / \nu}\left[\frac{1-1 / a}{1-t}\right] Q_{\lambda / \nu}\left[\frac{1-a q / t}{1-t}\right]=P_{\mu}\left[\frac{1-t^{k} / a}{1-t}\right] Q_{\lambda}\left[\frac{1-a q t^{\ell-1}}{1-t}\right] f_{\lambda, \mu}^{k, \ell}(a ; q, t),
$$

where $k \in \mathbb{N}$ and $\ell \in \mathbb{N} \cup\{\infty\}$ may be chosen arbitrarily, provided that $k \geqslant l(\lambda)$ and $\ell \geqslant l(\mu)$.

In 64] the right-hand side of (3.1) is stated with $\ell=k$. Of course, since the left-hand side does not depend on $k$ and $\ell$, the above form of the identity is not actually more general. Indeed,

$$
\begin{aligned}
& f_{\lambda, \mu}^{k, \ell}(a ; q, t) \\
& \quad=t^{-(k-l(\lambda))|\mu|} f_{\lambda, \mu}^{l(\lambda), l(\mu)}(a ; q, t) \prod_{i=1}^{l(\lambda)} \prod_{j=l(\mu)+1}^{\ell} \frac{\left(a q t^{j-i-1} ; q\right)_{\lambda_{i}}}{\left(a q t^{j-i} ; q\right)_{\lambda_{i}}} \prod_{i=l(\lambda)+1}^{k} \prod_{j=1}^{l(\mu)} \frac{\left(a q t^{j-i-1} ; q\right)_{-\mu_{j}}}{\left(a q t^{j-i} ; q\right)_{-\mu_{j}}} \\
& \quad=f_{\lambda, \mu}^{l(\lambda), l(\mu)}(a ; q, t) \frac{\left(a q t^{l(\mu)-1} ; q, t\right)_{\lambda}}{\left(a q t^{\ell-1} ; q, t\right)_{\lambda}} \frac{\left(t^{l(\lambda)} / a ; q, t\right)_{\mu}}{\left(t^{k} / a ; q, t\right)_{\mu}} .
\end{aligned}
$$

Substituting this in the right-hand side of (3.1) and using (2.19) yields the identity with $k$ and $\ell$ replaced by $l(\lambda)$ and $l(\mu)$. For later use we note that from the above and (2.19) it follows that

$$
f_{\lambda, \mu}^{k, \infty}(a ; q, t) Q_{\lambda}\left[\frac{1}{1-t}\right]=f_{\lambda, \mu}^{k, \ell}(a ; q, t) Q_{\lambda}\left[\frac{1-a q t^{\ell-1}}{1-t}\right],
$$

where $\ell$ is an arbitrary integer such that $\ell \geqslant l(\mu)$.

\footnotetext{
${ }^{5}$ For the relationship between $f_{\lambda, \mu}^{k, \ell}(a ; q, t)$ and Nekrasov-type functions, see [20, Equation (B.4)].
} 
For our next result we would like to specialise $a=t^{k-\ell}$ (for $k \leqslant \ell$ and $\ell$ finite) in (3.1). Potentially this could lead to problems with the double product on the right, and the following lemma serves to show that such a specialisation is in fact permitted provided the resulting double product is interpreted correctly.

Lemma 3.2. Let $\lambda$ and $\mu$ be partitions and $k, \ell \in \mathbb{N}$ such that $k \leqslant \ell$ and such that $k \geqslant l(\lambda)$ and $\ell \geqslant l(\mu)$. Then

$$
\lim _{b \rightarrow 1} f_{\lambda, \mu}^{k, \ell}\left(b t^{k-\ell} ; q, t\right)
$$

is well-defined. Furthermore a necessary and sufficient condition for the nonvanishing of this limit is

$$
\lambda_{i} \geqslant \mu_{i-k+\ell} \text { for } 1 \leqslant i \leqslant k .
$$

The inequalities (3.4) may conveniently be visualised as:

$$
\begin{array}{r}
\lambda_{1} \geqslant \lambda_{2} \geqslant \cdots \geqslant \lambda_{k} \geqslant \lambda_{k+1} \geqslant \cdots \geqslant 0 \\
\mathbb{V} \geqslant \mathbb{V}_{\mathbb{V}} \geqslant \mu_{\ell-k+1} \geqslant \mu_{\ell-k+2} \geqslant \cdots \geqslant \mu_{\ell} \geqslant \mu_{\ell+1} \geqslant \cdots \geqslant 0 .
\end{array}
$$

Remark. It is assumed in Lemma 3.2 that $q$ and $t$ are generic. For the Schur case $q=t$ the equation (3.4) has to be replaced by

$$
\lambda_{i}=\mu_{j_{i}}+i-j_{i}+\ell-k \text { for } 1 \leqslant i \leqslant k,
$$

where $1 \leqslant j_{1}<j_{2}<\cdots<j_{k} \leqslant \ell$.

Proof. To see that the limit is well-defined, note that for fixed $i$ the powers of $t$ in (3.3) are zero when $j=i-k+\ell+1$ in the numerator and $j=i-k+\ell$ in the denominator. Since $j \leqslant \ell$ this yields $k-\ell \leqslant i \leqslant k-1$ for the numerator and $k-\ell+1 \leqslant i \leqslant k$ for the denominator, with both the lower bounds automatically satisfied since $k \leqslant \ell$. Therefore, taking the product of the $t$-independent $q$-shifted factorials in (3.3) and making a shift in the indices yields

$$
\frac{\prod_{i=1}^{k-1}(b q ; q)_{\lambda_{i}-\mu_{i-k+\ell+1}}}{\prod_{i=1}^{k}(b q ; q)_{\lambda_{i}-\mu_{i-k+\ell}}}=\frac{1}{(b q ; q)_{\lambda_{k}-\mu_{\ell}}} \prod_{i=\ell-k+1}^{\ell-1}\left(b q^{1+\lambda_{i+\ell-k}-\mu_{i}} ; q\right)_{\mu_{i}-\mu_{i+1}} .
$$

Since $\mu$ is a partition, $\mu_{i} \geqslant \mu_{i+1}$, and hence the limit $b \rightarrow 1$ exists.

The vanishing of the limit (3.3) is completely determined by the vanishing of the right-hand side of (3.6) when $b \rightarrow 1$. Clearly the term $1 /(q ; q)_{\lambda_{k}-\mu_{\ell}}$ will vanish unless $\lambda_{k} \geqslant \mu_{\ell}$ by (2.4). In order for (3.6) to be nonvanishing, one of

$$
\begin{aligned}
& \lambda_{i+k-\ell} \geqslant \mu_{i}, \\
& \lambda_{i+k-\ell}<\mu_{i}=\mu_{i+1},
\end{aligned}
$$

must hold for each $i$ such that $\ell-k+1 \leqslant i \leqslant \ell-1$. Now assume that $\lambda_{k} \geqslant \mu_{\ell}$ and one of (3.7a) and (3.7b) holds. Consider the largest $i$ for which (3.7b) holds but (3.7a) does not. We cannot have $i=\ell-1$ as this would imply

$$
\lambda_{k}<\mu_{\ell-1}=\mu_{\ell}
$$

contradicting $\lambda_{k} \geqslant \mu_{\ell}$. Similarly, no such maximal $i$ exists with $i \leqslant \ell-1$ as we then would get

$$
\lambda_{i+k-\ell}<\mu_{i}=\mu_{i+1}
$$


However, as (3.7a) must now hold with $i \mapsto i+1$, this would give $\lambda_{i+k-\ell}<\lambda_{i+k-\ell+1}$, contradicting that $\lambda$ is a partition. Therefore we conclude that (3.7a) must hold for all $\ell-k+1 \leqslant i \leqslant \ell-1$. This is equivalent to the desired conditions by a shift of indices, and hence we are done.

By abuse of notation, we will write $f_{\lambda, \mu}^{k, \ell}\left(t^{k-\ell} ; q, t\right)$ instead of $\lim _{b \rightarrow 1} f_{\lambda, \mu}^{k, \ell}\left(b t^{k-\ell} ; q, t\right)$ in the following.

Corollary 3.3. Let $k, \ell \in \mathbb{N}$ such that $k \leqslant \ell$. Then, for partitions $\lambda, \mu$ such that $l(\lambda) \leqslant k$,

$$
\sum_{\nu} t^{-|\nu|} P_{\mu / \nu}\left[\frac{1-t^{\ell-k}}{1-t}\right] Q_{\lambda / \nu}\left[\frac{1-q t^{k-\ell-1}}{1-t}\right]=P_{\mu}\left[\frac{1-t^{\ell}}{1-t}\right] Q_{\lambda}\left[\frac{1-q t^{k-1}}{1-t}\right] f_{\lambda, \mu}^{k, \ell}\left(t^{k-\ell} ; q, t\right) .
$$

The above corollary is essentially [62, Theorem 4.1, $u=0$ ]. It should be noted that the condition $l(\mu) \leqslant \ell$ has been dropped in comparison with Proposition [3.1] and [62, Theorem 4.1], since both sides identically vanish when $l(\mu)>\ell$. To see this, note that the summand vanishes unless $\nu \subseteq \lambda$, $\nu \subseteq \mu$ and $\mu_{i-k+\ell} \leqslant \nu_{i}$ for all $i \geqslant 1$. This in particular implies that the summand vanishes unless $\mu_{i-k+\ell} \leqslant \lambda_{i}$ for all $i \geqslant 1$, in accordance with (3.4). When $i=k+1$ this yields $\mu_{\ell+1} \leqslant \lambda_{k+1}$. Now, since $l(\lambda) \leqslant k, \lambda_{k+1}=0$ so that $\mu_{\ell+1}=0$, i.e., $l(\mu) \leqslant \ell$. Clearly, the right-hand side has this same vanishing property.

3.2. $\mathbf{A}_{n}$ Cauchy-type identities. As we will see below, the Cauchy identity for Macdonald polynomials may be viewed as a discrete analogue of the AFLT integral. For the purpose of generalisation it is convenient to think of the Cauchy identity (2.16) as an identity for the root system $\mathrm{A}_{1}$ in which the two alphabets $X$ and $Y$ are attached to the single vertex of the corresponding Dynkin diagram:

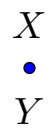

Extending this to $\mathrm{A}_{n}$, we consider sums of the form

$$
\sum_{\lambda^{(1)}, \ldots, \lambda^{(n)}} \prod_{r=1}^{n} P_{\lambda^{(r)}}\left[X^{(r)}\right] Q_{\lambda^{(r)}}\left[Y^{(r)}\right] \prod_{r=1}^{n-1} f_{\lambda^{(r)}, \lambda^{(r+1)}}^{k_{r}, k_{r+1}}\left(a_{r} ; q, t\right),
$$

\begin{tabular}{|c|c|c|c|}
\hline$X^{(1)}$ & $X^{(2)}$ & $X^{(r-1)}$ & $X^{(n-1)}$ \\
\hline$Y^{(1)}$ & $Y^{(2)}$ & $Y^{(r-1)}$ & $Y^{(n-1)}$ \\
\hline
\end{tabular}

where the functions $f_{\lambda^{(r)}, \lambda^{(r+1)}}^{k_{r}, k_{r+1}}$ represent the edges of the $\mathrm{A}_{n}$ Dynkin diagram:

In (3.8) we choose $k_{1} \leqslant k_{2} \leqslant \cdots \leqslant k_{n-1}$ to be nonnegative integers and $k_{n} \in \mathbb{N} \cup\{\infty\}$. Also, $a_{r}$ for $1 \leqslant r \leqslant n-2$ will be fixed as $a_{r}=t^{k_{r}-k_{r+1}}$, whereas $a_{n-1}$ is an indeterminate. In the sum (3.8) we also specialise the alphabets $X^{(2)}, \ldots, X^{(n)}$ and $Y^{(1)}, \ldots, Y^{(n-1)}$ as

$$
X^{(r+1)}=\frac{1-t^{k_{r}} / a_{r}}{1-t}, \quad Y^{(r)}=z_{r} \frac{t-a_{r} q t^{k_{r+1}}}{1-t} \quad \text { for } 1 \leqslant r \leqslant n-1,
$$

and fix the cardinalities of $X^{(1)}$ and $Y^{(n)}$ to be

$$
\left|X^{(1)}\right|=k_{1}, \quad\left|Y^{(n)}\right|=k_{n} .
$$


It should be noted that since $a_{r}=t^{k_{r}-k_{r+1}}$ for $r \neq n-1$ we have

$$
X^{(r)}=\frac{1-t^{k_{r}}}{1-t} \quad \text { for } 2 \leqslant r \leqslant n-1
$$

so that $\left|X^{(r)}\right|=k_{r}$ for all $1 \leqslant r \leqslant n-1$.

Recall the convention that $t^{k}:=0$ if $k=\infty$. Our first $\mathrm{A}_{n}$ Cauchy-type formula may then be stated as follows.

Theorem 3.4 ( $\mathrm{A}_{n}$ Cauchy-type formula I). Let $k_{1} \leqslant k_{2} \leqslant \cdots \leqslant k_{n-1}$ be nonnegative integers and $k_{n} \in \mathbb{N} \cup\{\infty\}$. Then for $a_{n-1}$ an indeterminate, $a_{r}:=t^{k_{r}-k_{r+1}}$ for $1 \leqslant r \leqslant n-2, X^{(1)}=$ $x_{1}+\cdots+x_{k_{1}}, Y^{(n)}=y_{1}+\cdots+y_{k_{n}}$ and $X^{(2)}, \ldots, X^{(n)}, Y^{(1)}, \ldots, Y^{(n-1)}$ as in (3.9), and

$$
W:=z_{1} \cdots z_{n-1} X^{(1)}+\sum_{r=1}^{n-1} z_{r+1} \cdots z_{n-1} \frac{1-1 / a_{r}}{1-t},
$$

we have

$$
\begin{aligned}
& \sum_{\lambda^{(1)}, \ldots, \lambda^{(n)}} \prod_{r=1}^{n} P_{\lambda^{(r)}}\left(\left[X^{(r)}\right] ; q, t\right) Q_{\lambda^{(r)} / \mu^{(r)}}\left(\left[Y^{(r)}\right] ; q, t\right) \prod_{r=1}^{n-1} f_{\lambda^{(r)}, \lambda^{(r+1)}}^{k_{r}, k_{r+1}}\left(a_{r} ; q, t\right) \\
&= P_{\mu^{(n)}}([W] ; q, t) \prod_{r=1}^{n-1}\left(\prod_{i=1}^{k_{1}} \frac{\left(a_{r} q z_{1} \cdots z_{r} x_{i} ; q\right)_{\infty}}{\left(t z_{1} \cdots z_{r} x_{i} ; q\right)_{\infty}} \prod_{j=1}^{k_{n}} \frac{\left(z_{r+1} \cdots z_{n-1} y_{j} / a_{r} ; q\right)_{\infty}}{\left(z_{r+1} \cdots z_{n-1} y_{j} ; q\right)_{\infty}}\right) \\
& \quad \times \prod_{i=1}^{k_{1}} \prod_{j=1}^{k_{n}} \frac{\left(t z_{1} \cdots z_{n-1} x_{i} y_{j} ; q\right)_{\infty}}{\left(z_{1} \cdots z_{n-1} x_{i} y_{j} ; q\right)_{\infty}} \prod_{1 \leqslant r<s \leqslant n-1} \prod_{i=1}^{k_{r+1}-k_{r}} \frac{\left(a_{s} q t^{i-1} z_{r+1} \cdots z_{s} ; q\right)_{\infty}}{\left(t^{i} z_{r+1} \cdots z_{s} ; q\right)_{\infty}}
\end{aligned}
$$

where $\mu^{(1)}, \ldots, \mu^{(n-1)}:=0$ and $\mu^{(n)}$ is an arbitrary partition.

For $n=1$ the theorem reduces to (2.16) with $(X, Y, \mu) \mapsto\left(X^{(1)}, Y^{(1)}, \mu^{(n)}\right)$, and for $n=2, k_{2}$ finite and $\mu^{(n)}=0$ it coincides with [64, Theorem 1.2]. When $n \geqslant 2$ there is some mild redundancy in (3.11) since the substitution $X^{(1)} \mapsto z_{1}^{-1} X^{(1)}$ eliminates any reference to $z_{1}$. We further remark that we do not know how to evaluate the left-hand side of (3.11) in closed-form if one (or more) of the $a_{r}$ for $1 \leqslant r \leqslant n-2$ is an indeterminate. Since $\left|X^{(r)}\right|=k_{r}$ for $1 \leqslant r \leqslant n-1$ the summand vanishes unless $l\left(\lambda^{(r)}\right) \leqslant k_{r}$ for this same range of $r$. If $a_{r}$ for some $r \leqslant n-2$ is an indeterminate, then $\lambda^{(r+1)}$ can have an arbitrarily large length, which prevents us from applying Proposition 3.1 in our proof. Requiring $a_{r}=t^{k_{r}-k_{r+1}}$ for $1 \leqslant r \leqslant n-1$ allows us to use Corollary [3.3 in place of the proposition. We are, however, allowed to keep $a_{n-1}$ an indeterminate since $Y^{(n)}$ is either a finite alphabet of cardinality $k_{n}$, or countably infinite, permitting us to apply Proposition 3.1. For more details we refer to the proof of the theorem contained in the next section.

There is a second, closely related, Cauchy-type identity, in which $k_{n}$ is finite and no longer corresponds to the cardinality of $Y^{(n)}$.

Corollary 3.5 ( $\mathrm{A}_{n}$ Cauchy-type formula II). Let $k_{1} \leqslant k_{2} \leqslant \cdots \leqslant k_{n}$ be nonnegative integers. Then for $a_{r}:=t^{k_{r}-k_{r+1}}$ for $1 \leqslant r \leqslant n-1, X^{(1)}=x_{1}+\cdots+x_{k_{1}}, Y^{(n)}=y_{1}+y_{2}+\cdots$ and $X^{(2)}, \ldots, X^{(n)}, Y^{(1)}, \ldots, Y^{(n-1)}$ as in (3.9), and

$$
W:=z_{1} \cdots z_{n-1} X^{(1)}+\sum_{r=1}^{n-1} z_{r+1} \cdots z_{n-1} \frac{1-t^{k_{r}-k_{r+1}}}{1-t}
$$


we have

$$
\begin{aligned}
& \sum_{\lambda^{(1)}, \ldots, \lambda^{(n)}} \prod_{r=1}^{n} P_{\lambda^{(r)}}\left(\left[X^{(r)}\right] ; q, t\right) Q_{\lambda^{(r)} / \mu^{(r)}}\left(\left[Y^{(r)}\right] ; q, t\right) \prod_{r=1}^{n-1} f_{\lambda^{(r)}, \lambda^{(r+1)}}^{k_{r}, k_{r+1}}\left(a_{r} ; q, t\right) \\
& =P_{\mu^{(n)}}([W] ; q, t) \prod_{r=1}^{n-1}\left(\prod_{i=1}^{k_{1}} \frac{\left(q t^{k_{r}-k_{r+1}} z_{1} \cdots z_{r} x_{i} ; q\right)_{\infty}}{\left(t z_{1} \cdots z_{r} x_{i} ; q\right)_{\infty}} \prod_{j \geqslant 1} \frac{\left(t^{k_{r+1}-k_{r}} z_{r+1} \cdots z_{n-1} y_{j} ; q\right)_{\infty}}{\left(z_{r+1} \cdots z_{n-1} y_{j} ; q\right)_{\infty}}\right) \\
& \quad \times \prod_{i=1}^{k_{1}} \prod_{j \geqslant 1} \frac{\left(t z_{1} \cdots z_{n-1} x_{i} y_{j} ; q\right)_{\infty}}{\left(z_{1} \cdots z_{n-1} x_{i} y_{j} ; q\right)_{\infty}} \prod_{1 \leqslant r<s \leqslant n-1} \prod_{i=1}^{k_{r+1}-k_{r}} \frac{\left(q t^{i+k_{s}-k_{s+1}-1} z_{r+1} \cdots z_{s} ; q\right)_{\infty}}{\left(t^{i} z_{r+1} \cdots z_{s} ; q\right)_{\infty}}
\end{aligned}
$$

where $\mu^{(1)}, \ldots, \mu^{(n-1)}:=0$ and $\mu^{(n)}$ is an arbitrary partition.

We note that in the above corollary the range for which (3.10) holds includes $r=n$. In particular $\left|X^{(r)}\right|=k_{r}$ for all $1 \leqslant r \leqslant n$. The corollary simplifies to the $\mathrm{A}_{n} q$-binomial theorem 63, Theorem 3.2] if we replace $Y^{(n)} \mapsto z_{n} t^{k_{n-1}}(1-a) /(1-t)$ and $z_{r} \mapsto z_{r} t^{k_{r-1}-1}$ for all $1 \leqslant r \leqslant n-1$ where $k_{0}:=0$.

Proof of Corollary 3.5. We take Theorem 3.4 with $k_{n}=\infty$. Then $Y^{(n)}=y_{1}+y_{2}+\cdots$ in accordance with the corollary. Moreover, by (3.2) and the fact that $\mu^{(n-1)}=0$,

$$
\begin{aligned}
& Q_{\lambda^{(n-1)} / \mu^{(n-1)}}\left[Y^{(n-1)}\right] f_{\lambda^{(n-1)}, \lambda^{(n)}}^{k_{n-1}, k_{n}}\left(a_{n-1} ; q, t\right) \\
& =Q_{\lambda^{(n-1)}}\left[\frac{z_{n} t}{1-t}\right] f_{\lambda^{(n-1)}, \lambda^{(n)}}^{k_{n-1}, \infty}\left(a_{n-1} ; q, t\right) \\
& =Q_{\lambda^{(n-1)}}\left[z_{n} \frac{t-a_{n-1} q t^{\hat{k}_{n}}}{1-t}\right] f_{\lambda^{(n-1)}, \lambda^{(n)}}^{k_{n-1} \hat{k}_{n}}\left(a_{n-1} ; q, t\right) \\
& =Q_{\lambda^{(n-1)} / \mu^{(n-1)}}\left[\hat{Y}^{(n-1)}\right] f_{\lambda^{(n-1)}, \lambda(n)}^{k_{n-1} \hat{k}_{n}}\left(a_{n-1} ; q, t\right) .
\end{aligned}
$$

Here $\hat{k}_{n}$ is an arbitrary integer such that $\hat{k}_{n} \geqslant l\left(\lambda^{(n)}\right)$ and $\hat{Y}^{(n-1)}:=z_{n}\left(t-a_{n-1} q t^{\hat{k}_{n}}\right) /(1-t)$, so that $\hat{Y}^{(n-1)}$ corresponds to $Y^{(n-1)}$ in (3.9) except that $k_{n}$ has been replaced by $\hat{k}_{n}$. Of course, since we are summing over all partitions $\lambda^{(n)}$ there exists no integer $\hat{k}_{n}$ such that $\hat{k}_{n} \geqslant l\left(\lambda^{(n)}\right)$ for all $\lambda^{(n)}$. To get around this problem we specialise $a_{n-1}=q^{k_{n-1}-\hat{k}_{n}}$. Then $X^{(n)}=\left(1-t^{\hat{k}_{n}}\right) /(1-t)$ of cardinality $\left|\hat{k}_{n}\right|$ so that without loss of generality we may assume that $l\left(\lambda^{(n)}\right) \leqslant \hat{k}_{n}$. Finally replacing $\hat{k}_{n}$ by $k_{n}$ completes the proof.

The proof of Theorem 1.2 actually requires a plethystically substituted version of the $\mu^{(n)}=0$ instance of (3.12) obtained by replacing $Y^{(n)} \mapsto Y^{(n)}+(c-d) /(1-t)$. This substitution can easily be carried out noting that the right-hand side of (3.12) without $P_{\mu^{(n)}}[W]$ is expressible in terms of $\sigma_{1}$ as

$$
\begin{gathered}
\sigma_{1}\left[\sum_{r=1}^{n-1}\left(t z_{1} \cdots z_{r} \frac{1-q t^{k_{r}-k_{r+1}-1}}{1-q} X^{(1)}+z_{r+1} \cdots z_{n-1} \frac{1-t^{k_{r+1}-k_{r}}}{1-q} Y^{(n)}\right)+\right. \\
\left.z_{1} \cdots z_{n-1} \frac{1-t}{1-q} X^{(1)} Y^{(n)}+\sum_{1 \leqslant r<s \leqslant n-1} t z_{r+1} \cdots z_{s} \frac{\left(1-q t^{k_{s}-k_{s+1}-1}\right)\left(1-t^{k_{r+1}-k_{r}}\right)}{(1-q)(1-t)}\right] .
\end{gathered}
$$


Corollary 3.6. With the same conditions as in Corollary [3.5,

$$
\begin{aligned}
& \sum_{\lambda^{(1)}, \ldots, \lambda^{(n)}} P_{\lambda^{(n)}}\left(\left[X^{(n)}\right] ; q, t\right) Q_{\lambda^{(n)}}\left(\left[Y^{(n)}+\frac{c-d}{1-t}\right] ; q, t\right) \\
& \times \prod_{r=1}^{n-1}\left(P_{\lambda^{(r)}}\left(\left[X^{(r)}\right] ; q, t\right) Q_{\lambda^{(r)}}\left(\left[Y^{(r)}\right] ; q, t\right) f_{\lambda^{(r)}, \lambda^{(r+1)}}^{k_{r}, k_{r+1}}\left(a_{r} ; q, t\right)\right) \\
& =\prod_{r=1}^{n-1}\left(\prod_{i=1}^{k_{1}} \frac{\left(q t^{k_{r}-k_{r+1}} z_{1} \cdots z_{r} x_{i} ; q\right)_{\infty}}{\left(t z_{1} \cdots z_{r} x_{i} ; q\right)_{\infty}} \prod_{j \geqslant 1} \frac{\left(t^{k_{r+1}-k_{r}} z_{r+1} \cdots z_{n-1} y_{j} ; q\right)_{\infty}}{\left(z_{r+1} \cdots z_{n-1} y_{j} ; q\right)_{\infty}}\right) \\
& \times \prod_{i=1}^{k_{1}} \prod_{j \geqslant 1} \frac{\left(t z_{1} \cdots z_{n-1} x_{i} y_{j} ; q\right)_{\infty}}{\left(z_{1} \cdots z_{n-1} x_{i} y_{j} ; q\right)_{\infty}} \prod_{i=1}^{k_{1}} \frac{\left(d z_{1} \cdots z_{n-1} x_{i} ; q\right)_{\infty}}{\left(c z_{1} \cdots z_{n-1} x_{i} ; q\right)_{\infty}} \\
& \times \prod_{r=1}^{n-1} \prod_{i=1}^{k_{r+1}-k_{r}} \frac{\left(d z_{r+1} \cdots z_{n-1} t^{i-1} ; q\right)_{\infty}}{\left(c z_{r+1} \cdots z_{n-1} t^{i-1} ; q\right)_{\infty}} \prod_{1 \leqslant r<s \leqslant n-1} \prod_{i=1}^{k_{r+1}-k_{r}} \frac{\left(q t^{i+k_{s}-k_{s+1}-1} z_{r+1} \cdots z_{s} ; q\right)_{\infty}}{\left(t^{i} z_{r+1} \cdots z_{s} ; q\right)_{\infty}}
\end{aligned}
$$

3.3. Proof of Theorem 3.4. We define two families of auxiliary alphabets $\left\{X^{(r, m)}\right\}_{0 \leqslant m<r \leqslant n}$ and $\left\{Z^{(r)}\right\}_{r=1}^{n}$ as

$$
X^{(r, m)}:= \begin{cases}z_{1} \cdots z_{m} X^{(1)}+\sum_{u=1}^{m} z_{u+1} \cdots z_{m} \frac{1-1 / a_{u}}{1-t} & \text { if } r=m+1 \\ \frac{1-1 / a_{r-1}}{1-t} & \text { otherwise }\end{cases}
$$

and

$$
Z^{(r)}:= \begin{cases}Y^{(n)} & \text { if } r=n, \\ z_{r} \frac{t-a_{r} q}{1-t} & \text { otherwise. }\end{cases}
$$

The first family satisfies the simple recursion

$$
z_{m+1} X^{(m+1, m)}+X^{(m+2, m)}=X^{(m+2, m+1)} .
$$

Lemma 3.7. For $n, m$ integers such that $0 \leqslant m \leqslant n-1$, and $\nu^{(n)}$ a partition, define

$$
\begin{aligned}
g_{m}:= & \prod_{r=1}^{m} \prod_{i=1}^{k_{1}} \frac{\left(a_{r} q z_{1} \cdots z_{r} x_{i} ; q\right)_{\infty}}{\left(t z_{1} \cdots z_{r} x_{i} ; q\right)_{\infty}} \prod_{1 \leqslant r<s \leqslant m} \prod_{i=1}^{k_{r+1}-k_{r}} \frac{\left(a_{s} q t^{i-1} z_{r+1} \cdots z_{s} ; q\right)_{\infty}}{\left(t^{i} z_{r+1} \cdots z_{s} ; q\right)_{\infty}} \\
& \times \sum_{\nu^{(m+1)}, \ldots, \nu^{(n-1)}} \prod_{r=m+1}^{n}\left(z_{r}^{\left|\nu^{(r)}\right|} \sum_{\lambda^{(r)}} P_{\lambda^{(r)} / \nu^{(r-1)}}\left(\left[X^{(r, m)}\right] ; q, t\right) Q_{\lambda^{(r)} / \nu^{(r)}}\left(\left[Z^{(r)}\right] ; q, t\right)\right),
\end{aligned}
$$

where $\nu^{(m)}:=0$. Then $g_{m}=g_{m+1}$ for $0 \leqslant m \leqslant n-2$.

Proof. Since $\nu^{(m)}:=0$, the sum over $\lambda^{(m+1)}$ in (3.14) is of the form (2.16) with

$$
(X, Y, \lambda, \mu) \mapsto\left(X^{(m+1, m)}, Z^{(m+1)}, \lambda^{(m+1)}, \nu^{(m+1)}\right)
$$


and hence equates to

$$
P_{\nu^{(m+1)}}\left[X^{(m+1, m)}\right] \sigma_{1}\left[\frac{1-t}{1-q} X^{(m+1, m)} Z^{(m+1)}\right] .
$$

Since $0 \leqslant m \leqslant n-2$, it follows that

$$
\begin{aligned}
\sigma_{1} & {\left[\frac{1-t}{1-q} X^{(m+1, m)} Z^{(m+1)}\right] } \\
& =\sigma_{1}\left[z_{1} \cdots z_{m+1} \frac{t-a_{m+1} q}{1-q} X^{(1)}+\sum_{r=1}^{m} z_{r+1} \cdots z_{m+1} \frac{\left(1-t^{k_{r+1}-k_{r}}\right)\left(t-a_{m+1} q\right)}{(1-t)(1-q)}\right] \\
& =\prod_{i=1}^{k_{1}} \sigma_{z_{1} \cdots z_{m+1}}\left[\frac{t-a_{m+1} q}{1-q} x_{i}\right] \prod_{r=1}^{m} \prod_{i=1}^{k_{r+1}-k_{r}} \sigma_{z_{r+1} \cdots z_{m+1}}\left[\frac{t^{i-1}\left(t-a_{m+1} q\right)}{1-q}\right] \\
& =\prod_{i=1}^{k_{1}} \frac{\left(a_{m+1} q z_{1} \cdots z_{m+1} x_{i} ; q\right)_{\infty}}{\left(t z_{1} \cdots z_{m+1} x_{i} ; q\right)_{\infty}} \prod_{r=1}^{m} \prod_{i=1}^{k_{r+1}-k_{r}} \frac{\left(a_{m+1} q t^{i-1} z_{r+1} \cdots z_{m+1} ; q\right)_{\infty}}{\left(t^{i} z_{r+1} \cdots z_{m+1} ; q\right)_{\infty}}
\end{aligned}
$$

where the second equality follows from (2.13) and the last equality from (2.14). As a result,

$$
\begin{aligned}
g_{m}= & \prod_{r=1}^{m+1} \prod_{i=1}^{k_{1}} \frac{\left(a_{r} q z_{1} \cdots z_{r} x_{i} ; q\right)_{\infty}}{\left(t z_{1} \cdots z_{r} x_{i} ; q\right)_{\infty}} \prod_{1 \leqslant r<s \leqslant m+1} \prod_{i=1}^{k_{r+1}-k_{r}} \frac{\left(a_{s} q t^{i-1} z_{r+1} \cdots z_{s} ; q\right)_{\infty}}{\left(t^{i} z_{r+1} \cdots z_{s} ; q\right)_{\infty}} \\
\times & \left\{P_{\nu^{(m+1)}}\left[z_{m+1} X^{(m+1, m)}\right]\right. \\
& \left.\times \prod_{r=m+2}^{n}\left(z_{r}^{\left|\nu^{(r)}\right|} \sum_{\lambda^{(r)}} P_{\lambda^{(r)} / \nu^{(r-1)}}\left[X^{(r, m)}\right] Q_{\lambda^{(r)} / \nu^{(r)}}\left[Z^{(r)}\right]\right)\right\} .
\end{aligned}
$$

After interchanging the order of the sum over $\nu^{(m+1)}$ with those over the $\lambda^{(r)}$, the former can be summed using (2.15) with

$$
(X, Y, \lambda, \mu) \mapsto\left(X^{(m+2, m)}, z_{m+1} X^{(m+1, m)}, \lambda^{(m+2)}, \nu^{(m+1)}\right) .
$$

Thanks to the recursion (3.13) this yields $P_{\lambda^{(m+2)}}\left[X^{(m+2, m+1)}\right]$, resulting in $g_{m}=g_{m+1}$.

We are now ready to prove Theorem 3.4. As a first step we eliminate $f_{\lambda^{(r)}, \lambda^{(r+1)}}^{k_{r}, k_{r+1}}\left(a_{r} ; q, t\right)$ from the summand in (3.11) by applying Corollary 3.3 with

$$
(\lambda, \mu, \nu, k, \ell) \mapsto\left(\lambda^{(r)}, \lambda^{(r+1)}, \nu^{(r)}, k_{r}, k_{r+1}\right) \quad \text { for } 1 \leqslant r \leqslant n-2
$$

and Proposition 3.1 with

$$
(a, \lambda, \mu, \nu, k, \ell) \mapsto\left(a_{n-1}, \lambda^{(n-1)}, \lambda^{(n)}, \nu^{(n-1)}, k_{n-1}, k_{n}\right) \quad \text { for } r=n-1 .
$$

Then

$$
\begin{gathered}
\sum_{\lambda^{(1)}, \ldots, \lambda^{(n)}} \prod_{r=1}^{n} P_{\lambda^{(r)}}\left[X^{(r)}\right] Q_{\lambda^{(r)} / \mu^{(r)}}\left[Y^{(r)}\right] \prod_{r=1}^{n-1} f_{\lambda^{(r)}, \lambda^{(r+1)}}^{k_{r}, k_{r+1}}\left(a_{r} ; q, t\right) \\
=\sum_{\lambda^{(1)}, \ldots, \lambda^{(n)}} \sum_{\nu^{(1)}, \ldots, \nu^{(n-1)}} P_{\lambda^{(1)}}\left[X^{(1)}\right] Q_{\lambda^{(n)} / \mu^{(n)}}\left[Y^{(n)}\right]
\end{gathered}
$$




$$
\begin{aligned}
& \times \prod_{r=1}^{n-1} z_{r}^{\left|\lambda^{(r)}\right|} Q_{\lambda^{(r)} / \nu^{(r)}}\left[\frac{t-a_{r} q}{1-t}\right] P_{\lambda^{(r+1)} / \nu^{(r)}}\left[\frac{1-1 / a_{r}}{1-t}\right] \\
= & \left.\sum_{\nu^{(1)}, \ldots, \nu^{(r-1)}} \prod_{r=1}^{n}\left(z_{r}^{\left|\nu^{(r)}\right|} \sum_{\lambda^{(r)}} P_{\lambda^{(r)} / \nu^{(r-1)}}\left[X^{(r, 0)}\right] Q_{\lambda^{(r)} / \nu^{(r)}}\left[Z^{(r)}\right]\right)\right|_{z_{n}=1, \nu^{(n)}=\mu^{(n)}} \\
= & \left.g_{0}\right|_{z_{n}=1, \nu^{(n)}=\mu^{(n)}},
\end{aligned}
$$

where in the fourth line $\nu^{(0)}:=0$, and where $g_{0}$ is defined in (3.14). Using Lemma 3.7 we may replace $g_{0}$ by $g_{n-1}$, leading to

$$
\begin{aligned}
\sum_{\lambda^{(1)}, \ldots, \lambda^{(n)}} & \prod_{r=1}^{n} P_{\lambda^{(r)}}\left[X^{(r)}\right] Q_{\lambda^{(r)} / \mu^{(r)}}\left[Y^{(r)}\right] \prod_{r=1}^{n-1} f_{\lambda^{(r)}, \lambda^{(r+1)}}^{k_{r}, k_{r+1}}\left(a_{r} ; q, t\right) \\
= & \prod_{r=1}^{n-1} \prod_{i=1}^{k_{1}} \frac{\left(a_{r} q z_{1} \cdots z_{r} x_{i} ; q\right)_{\infty}}{\left(t z_{1} \cdots z_{r} x_{i} ; q\right)_{\infty}} \prod_{1 \leqslant r<s \leqslant n-1} \prod_{i=1}^{k_{r+1}-k_{r}} \frac{\left(a_{s} q t^{i-1} z_{r+1} \cdots z_{s} ; q\right)_{\infty}}{\left(t^{i} z_{r+1} \cdots z_{s} ; q\right)_{\infty}} \\
& \times \sum_{\lambda^{(n)}} P_{\lambda^{(n)}}\left[X^{(n, n-1)}\right] Q_{\lambda^{(n)} / \mu^{(n)}}\left[Y^{(n)}\right] .
\end{aligned}
$$

The final sum on the right can be carried out by (2.16) with

$$
(X, Y, \lambda, \mu) \mapsto\left(X^{(n, n-1)}, Y^{(n)}, \lambda^{(n)}, \mu^{(n)}\right) .
$$

Since $W=X^{(n, n-1)}$ and

$$
\begin{aligned}
\sigma_{1}\left[\frac{1-t}{1-q} X^{(n, n-1)} Y^{(n)}\right] & =\sigma_{1}\left[z_{1} \cdots z_{n-1} \frac{1-t}{1-q} X^{(1)} Y^{(n)}+\sum_{r=1}^{n-1} z_{r+1} \cdots z_{n-1} \frac{1-1 / a_{r}}{1-q} Y^{(n)}\right] \\
& =\prod_{i=1}^{k_{1}} \prod_{j=1}^{k_{n}} \frac{\left(t z_{1} \cdots z_{n-1} x_{i} y_{j} ; q\right)_{\infty}}{\left(z_{1} \cdots z_{n-1} x_{i} y_{j} ; q\right)_{\infty}} \prod_{r=1}^{n-1} \prod_{j=1}^{k_{n}} \frac{\left(z_{r+1} \cdots z_{n-1} y_{j} / a_{r} ; q\right)_{\infty}}{\left(z_{r+1} \cdots z_{n-1} y_{j} ; q\right)_{\infty}}
\end{aligned}
$$

the right-hand side of the theorem results.

\section{The $\mathrm{A}_{n}$ AFLT integral}

In this section we first give a description of the domain on integration of the $\mathrm{A}_{n}$ AFLT integral (1.13) and then apply the $\mathrm{A}_{n}$ Cauchy identity of Corollary 3.6 to prove this integral. At the end of the section we give a companion to the AFLT integral based on Theorem 3.4

4.1. The domain $C_{\gamma}^{k_{1}, \ldots, k_{n}}[\mathbf{0 , 1}]$. The domain of integration $C_{\gamma}^{k_{1}, \ldots, k_{n}}[0,1]$ of the integral (1.10) takes the form of a chain in the usual sense of algebraic topology. Since this chain is the same as that of the $\mathrm{A}_{n}$ Selberg integral of [63, Theorem 1.2] (see also [56] where this chain first appeared in the case of $\mathrm{A}_{2}$ ), we refer to [63] for the details of exactly how it arises in the course of the proof presented in Section 4.2.

For $n=1$ the domain $C_{\gamma}^{k_{1}}[0,1]$ is the $k_{1}$-simplex given in (1.14). In order to describe $C_{\gamma}^{k_{1}, \ldots, k_{n}}[0,1]$ for $n \geqslant 2$, we first consider $D^{k_{1}, \ldots, k_{n}}[0,1] \subseteq[0,1]^{k_{1}+\cdots+k_{n}}$ as the set of points

$$
\left(t^{(1)}, t^{(2)}, \ldots, t^{(n)}\right)=\left(t_{1}^{(1)}, \ldots, t_{k_{1}}^{(1)}, t_{1}^{(2)}, \ldots, t_{k_{2}}^{(2)}, \ldots, t_{1}^{(n)}, \ldots, t_{k_{n}}^{(n)}\right) \in[0,1]^{k_{1}+\cdots+k_{n}}
$$


subject to

$$
0<t_{1}^{(r)}<\cdots<t_{k_{r}}^{(r)}<1 \quad \text { for } 1 \leqslant r \leqslant n
$$

and

$$
t_{i}^{(r)}<t_{i-k_{r}+k_{r+1}}^{(r+1)} \quad \text { for } 1 \leqslant i \leqslant k_{r}, 1 \leqslant r \leqslant n-1 .
$$

Following (3.5) this may be visualised as

$$
\begin{aligned}
0 & <t_{1}^{(r)}<t_{2}^{(r)}<\cdots<t_{k_{r}}^{(r)}<1 \\
0<t_{1}^{(r+1)}<\cdots<t_{k_{r+1}-k_{r}+1}^{(r+1)}<t_{k_{r+1}-k_{r}+2}^{(r+1)}<\cdots<t_{k_{r+1}}^{(r+1)}<1 . & <
\end{aligned}
$$

We need to consider all possible total orderings between the integration variables $t^{(r)}$ and $t^{(r+1)}$ consistent with the above partial order. Each such total ordering may be described by a map

$$
M_{r}:\left\{1, \ldots, k_{r}\right\} \longrightarrow\left\{1, \ldots, k_{r+1}\right\}
$$

such that $M_{r}(i) \leqslant M_{r}(i+1)$ and $1 \leqslant M_{r}(i) \leqslant i+k_{r+1}-k_{r}$, so that

$$
t_{M_{r}(i)-1}^{(r+1)}<t_{i}^{(r)}<t_{M_{r}(i)}^{(r+1)}
$$

where $t_{0}^{(r+1)}:=0$. In view of this we define the sets

$$
D_{M_{1}, \ldots, M_{n-1}}^{k_{1}, \ldots, k_{n}} \subseteq D^{k_{1}, \ldots, k_{n}}[0,1]
$$

by requiring that (4.1) holds for fixed admissible maps $M_{1}, \ldots, M_{n-1}$. Then $D^{k_{1}, \ldots, k_{n}}$ can be written as the chain

$$
D^{k_{1}, \ldots, k_{n}}[0,1]=\sum_{M_{1}, \ldots, M_{n-1}} D_{M_{1}, \ldots, M_{n-1}}^{k_{1}, \ldots, k_{n}}[0,1]
$$

where the sum is over all admissible maps $M_{1}, \ldots, M_{n-1}$. Analytically continuing the weight function

$$
F_{M_{1}, \ldots, M_{n-1}}^{k_{1}, \ldots, k_{n}}(\gamma):=\prod_{r=1}^{n-1} \prod_{i=1}^{k_{r}} \frac{\sin \left(\pi\left(i+k_{r+1}-k_{r}-M_{r}(i)+1\right) \gamma\right)}{\sin \left(\pi\left(i+k_{r+1}-k_{r}\right) \gamma\right)} \quad \text { for } \gamma \in \mathbb{C} \backslash \mathbb{Z},
$$

to include $\gamma=0$, the chain $C_{\gamma}^{k_{1}, \ldots, k_{n}}[0,1]$ is defined as

$$
C_{\gamma}^{k_{1}, \ldots, k_{n}}[0,1]:=\sum_{M_{1}, \ldots, M_{n-1}} F_{M_{1}, \ldots, M_{n-1}}^{k_{1}, \ldots, k_{n}}(\gamma) D_{M_{1}, \ldots, M_{n-1}}^{k_{1}, \ldots, k_{n}}[0,1] .
$$

Note that it follows from the above that

$$
C_{\gamma}^{0, k_{2}, \ldots, k_{n}}[0,1]=C_{\gamma}^{k_{2}, \ldots, k_{n}}[0,1] .
$$

4.2. Proof of Theorem 1.2. We begin with the identity of Corollary 3.6, where we note that the alphabets $X^{(1)}$ and $Y^{(n)}$ contain $k_{1}$ variables and countably many variables respectively. We now fix a nonnegative integer $m$ and set $Y_{i}^{(n)}=0$ for $i>m$. Next we fix a pair of partitions $\lambda \in \mathscr{P}_{k_{1}}$ and $\mu \in \mathscr{P}_{m}$, and carry out the specialisation

$$
\left(X^{(1)}, Y^{(n)}, c, d\right) \mapsto\left(\langle\lambda\rangle_{k_{1}}, b z_{n} t^{1-m}\langle\mu\rangle_{m}, z_{n} t, b z_{n} t^{1-m}\right) .
$$


Also replacing $\lambda^{(1)}, \ldots, \lambda^{(n)}$ by $\nu^{(1)}, \ldots, \nu^{(n)}$, this leads to the identity

$$
\begin{aligned}
\sum_{\nu^{(1)}, \ldots, \nu^{(n)}} & P_{\nu^{(1)}}\left[\langle\lambda\rangle_{k_{1}}\right] Q_{\nu^{(n)}}\left[b t^{-m}\langle\mu\rangle_{m}+\frac{1-b t^{-m}}{1-t}\right] \\
& \times \prod_{r=1}^{n}\left(t z_{r}\right)^{\left|\nu^{(r)}\right|} \prod_{r=1}^{n-1}\left(P_{\nu^{(r+1)}}\left[\frac{1-t^{k_{r+1}}}{1-t}\right] Q_{\nu^{(r)}}\left[\frac{1-q t^{k_{r}-1}}{1-t}\right] f_{\nu^{(r)}, \nu^{(r+1)}}^{k_{r}, k_{r+1}}\left(t^{k_{r}-k_{r+1}} ; q, t\right)\right) \\
= & \prod_{r=1}^{n-1}\left(\prod_{i=1}^{k_{1}} \frac{\left(z_{1} \cdots z_{r} q^{\lambda_{i}+1} t^{k_{1}+k_{r}-k_{r+1}-i} ; q\right)_{\infty}}{\left(z_{1} \cdots z_{r} q^{\lambda_{i}} t^{k_{1}-i+1} ; q\right)_{\infty}} \prod_{j=1}^{m} \frac{\left(b z_{r+1} \cdots z_{n} q^{\mu_{j}} t^{k_{r+1}-k_{r}-j} ; q\right)_{\infty}}{\left(b z_{r+1} \cdots z_{n} q^{\mu_{j}} t^{-j} ; q\right)_{\infty}}\right) \\
& \times \prod_{i=1}^{k_{1}} \prod_{j=1}^{m} \frac{\left(b z_{1} \cdots z_{n} q^{\lambda_{i}+\mu_{j}} t^{k_{1}+1-i-j} ; q\right)_{\infty}}{\left(b z_{1} \cdots z_{n} q^{\lambda_{i}+\mu_{j}} t^{k_{1}-i-j} ; q\right)_{\infty}} \prod_{i=1}^{k_{1}} \frac{\left(b z_{1} \cdots z_{n-1} q^{\lambda_{i}} t^{k_{1}-m-i} ; q\right)_{\infty}}{\left(z_{1} \cdots z_{n-1} q^{\lambda_{i}} t^{k_{1}-i} ; q\right)_{\infty}} \\
& \times \prod_{r=1}^{n-1} \prod_{i=1}^{k_{r+1}-k_{r}} \frac{\left(b z_{r+1} \cdots z_{n-1} t^{i-m-1} ; q\right)_{\infty}}{\left(z_{r+1} \cdots z_{n-1} t^{i-1} ; q\right)_{\infty}} \prod_{1 \leqslant r<s \leqslant n-1} \prod_{i=1}^{k_{r+1}-k_{r}} \frac{\left(q t^{k_{s}-k_{s+1}+i-1} z_{r+1} \cdots z_{s} ; q\right)_{\infty}}{\left(t^{i} z_{r+1} \cdots z_{s} ; q\right)_{\infty}}
\end{aligned}
$$

where we have dropped $a_{r}$ in favour of $t^{k_{r}-k_{r+1}}$ in comparison with Corollary 3.6. By virtue of the evaluation symmetry (2.21) and the generalised evaluation symmetry of Lemma 2.2, we have

$$
P_{\nu^{(1)}}\left[\langle\lambda\rangle_{k_{1}}\right]=\frac{P_{\nu^{(1)}}\left[\frac{1-t^{k_{1}}}{1-t}\right]}{P_{\lambda}\left[\frac{1-t^{k_{1}}}{1-t}\right]} P_{\lambda}\left[\left\langle\nu^{(1)}\right\rangle_{k_{1}}\right]
$$

and

$$
Q_{\nu^{(n)}}\left[b t^{-m}\langle\mu\rangle_{m}+\frac{1-b t^{-m}}{1-t}\right]=\frac{Q_{\nu^{(n)}}\left[\frac{1-b}{1-t}\right]}{P_{\mu}\left[\frac{1-b}{1-t}\right]} P_{\mu}\left[b t^{-k_{n}}\left\langle\nu^{(n)}\right\rangle_{k_{n}}+\frac{1-b t^{-k_{n}}}{1-t}\right]
$$

effectively allowing us to interchange the roles of $\nu^{(1)}, \nu^{(n)}$ and $\lambda, \mu$ in the summand. Carrying this out and multiplying both sides by

$$
P_{\lambda}\left[\frac{1-t^{k_{1}}}{1-t}\right] P_{\mu}\left[\frac{1-b}{1-t}\right]
$$

the left-hand side of the above identity becomes

$$
\begin{aligned}
\sum_{\nu^{(1)}, \ldots, \nu^{(n)}} P_{\lambda}\left[\left\langle\nu^{(1)}\right\rangle_{k_{1}}\right] P_{\mu}\left[b t^{-k_{n}}\left\langle\nu^{(n)}\right\rangle_{k_{n}}+\frac{1-b t^{-k_{n}}}{1-t}\right](b ; q, t)_{\nu^{(n)}} \\
\quad \times \prod_{r=1}^{n} \frac{\left(t z_{r}\right)^{\left|\nu^{(r)}\right|} t^{2 n\left(\nu^{(r)}\right)}\left(t^{k_{r}} ; q, t\right)_{\nu^{(r)}}}{c_{\nu^{(r)}}(q, t) c_{\nu^{(r)}}^{\prime}(q, t)} \prod_{r=1}^{n-1}\left(q t^{k_{r}-1} ; q, t\right)_{\nu^{(r)}} f_{\nu^{(r)}, \nu^{(r+1)}}^{k_{r}, k_{r+1}}\left(t^{k_{r}-k_{r+1}} ; q, t\right),
\end{aligned}
$$

where we have also used the specialisation formulas (2.18) and (2.19). The corresponding righthand side is as before, except for the additional factor (4.4). Next we use (2.5) and (2.6) in the summand, make the further substitutions

$$
b \mapsto q^{\beta+\left(k_{n}-1\right) \gamma}, \quad t \mapsto q^{\gamma} \quad \text { and } \quad z_{r} \mapsto q^{\alpha_{r}-\gamma} \quad \text { for } 1 \leqslant r \leqslant n,
$$


and introduce auxiliary variables $\left(t^{(1)}, \ldots, t^{(n)}\right)$ as $t_{i}^{(r)}:=q^{\nu_{i}^{(r)}}+\left(k_{r}-i\right) \gamma$. To more simply express the resulting identity we introduce some additional notation, and for alphabets $t=\left(t_{1}, \ldots, t_{k}\right)$, $s=\left(s_{1}, \ldots, s_{\ell}\right)$ define

$$
\Delta_{\gamma}(t ; q):=\prod_{1 \leqslant i<j \leqslant k} t_{j}^{2 \gamma}\left(1-t_{i} / t_{j}\right)\left(q^{1-\gamma} t_{i} / t_{j} ; q\right)_{2 \gamma-1}
$$

and

$$
\Delta_{\gamma}(t, s ; q):=\prod_{i=1}^{k} \prod_{j=1}^{\ell} s_{j}^{-\gamma}\left(q t_{i} / s_{j} ; q\right)_{-\gamma} .
$$

After multiplying through by $(1-q)^{k_{1}+\cdots+k_{n}}$, this yields

$$
\begin{aligned}
& (1-q)^{k_{1}+\cdots+k_{n}} \sum_{\nu^{(1)}, \ldots, \nu^{(n)}} P_{\lambda}\left(t^{(1)} ; q, q^{\gamma}\right) P_{\mu}\left(\left[q^{\beta-\gamma} t^{(n)}+\frac{1-q^{\beta-\gamma}}{1-q^{\gamma}}\right] ; q, q^{\gamma}\right) \\
& \times \prod_{r=1}^{n}\left(\Delta_{\gamma}\left(t^{(r)} ; q\right) \prod_{i=1}^{k_{r}}\left(t_{i}^{(r)}\right)^{\alpha_{r}}\left(q t_{i}^{(r)} ; q\right)_{\beta_{r}-1}\right) \prod_{r=1}^{n-1} \Delta_{\gamma}\left(t^{(r)}, t^{(r+1)} ; q\right) \\
& =q^{\gamma \sum_{r=1}^{n}\left(\alpha_{r}\left(\begin{array}{c}
k_{r} \\
2
\end{array}\right)+2 \gamma\left(\begin{array}{c}
k_{r} \\
3
\end{array}\right)\right)-\gamma^{2} \sum_{r=1}^{n-1} k_{r}\left(\begin{array}{c}
k_{r+1} \\
2
\end{array}\right)} \\
& \times P_{\lambda}\left(\left[\frac{1-q^{k_{1} \gamma}}{1-q^{\gamma}}\right] ; q, q^{\gamma}\right) P_{\mu}\left(\left[\frac{1-q^{\beta+\left(k_{n}-1\right) \gamma}}{1-q^{\gamma}}\right] ; q, q^{\gamma}\right) \\
& \times \prod_{r=1}^{n-1} \prod_{i=1}^{k_{r}} \frac{\Gamma_{q}\left(1+\left(i-k_{r+1}-1\right) \gamma\right) \Gamma_{q}(i \gamma)}{\Gamma_{q}(\gamma)} \prod_{i=1}^{k_{n}} \frac{\Gamma_{q}(\beta+(i-1) \gamma) \Gamma_{q}(i \gamma)}{\Gamma_{q}(\gamma)} \\
& \times \prod_{1 \leqslant r<s \leqslant n-1} \prod_{i=1}^{k_{r+1}-k_{r}} \frac{\Gamma_{q}\left(\alpha_{r+1}+\cdots+\alpha_{s}+(r-s+i) \gamma\right)}{\Gamma_{q}\left(1+\alpha_{r+1}+\cdots+\alpha_{s}+\left(k_{s}-k_{s+1}+i+r-s-1\right) \gamma\right)} \\
& \times \prod_{r=1}^{n-1}\left(\prod_{i=1}^{k_{r+1}-k_{r}} \frac{\Gamma_{q}\left(\alpha_{r+1}+\cdots+\alpha_{n}+(r-n+i) \gamma\right)}{\Gamma_{q}\left(\alpha_{r+1}+\cdots+\alpha_{n}+\beta+\left(k_{n}-m+r-n+i-1\right) \gamma\right)}\right. \\
& \times \prod_{i=1}^{k_{1}} \frac{\Gamma_{q}\left(\alpha_{1}+\cdots+\alpha_{r}+\left(k_{1}-r-i+1\right) \gamma+\lambda_{i}\right)}{\Gamma_{q}\left(1+\alpha_{1}+\cdots+\alpha_{r}+\left(k_{1}+k_{r}-k_{r+1}-r-i\right) \gamma+\lambda_{i}\right)} \\
& \left.\times \prod_{j=1}^{m} \frac{\Gamma_{q}\left(\alpha_{r+1}+\cdots+\alpha_{n}+\beta+\left(k_{n}+r-n-j\right) \gamma+\mu_{j}\right)}{\Gamma_{q}\left(\alpha_{r+1}+\cdots+\alpha_{n}+\beta+\left(k_{r+1}-k_{r}+k_{n}+r-n-j\right) \gamma+\mu_{j}\right)}\right) \\
& \times \prod_{i=1}^{k_{1}} \frac{\Gamma_{q}\left(\alpha_{1}+\cdots+\alpha_{n}+\left(k_{1}-n-i+1\right) \gamma+\lambda_{i}\right)}{\Gamma_{q}\left(\alpha_{1}+\cdots+\alpha_{n}+\beta+\left(k_{1}+k_{n}-m-n-i\right) \gamma+\lambda_{i}\right)} \\
& \times \prod_{i=1}^{k_{1}} \prod_{j=1}^{m} \frac{\Gamma_{q}\left(\alpha_{1}+\cdots+\alpha_{n}+\beta+\left(k_{1}+k_{n}-n-i-j\right) \gamma+\lambda_{i}+\mu_{j}\right)}{\Gamma_{q}\left(\alpha_{1}+\cdots+\alpha_{n}+\beta+\left(k_{1}+k_{n}-n-i-j+1\right) \gamma+\lambda_{i}+\mu_{j}\right)},
\end{aligned}
$$

where $\beta_{1}=\cdots=\beta_{n-1}:=1$ and $\beta_{n}:=\beta$. The above is a restricted $q$-integral over the domain $D^{k_{1}, \ldots, k_{n}}[0,1]$. To complete the proof we divide the above identity by its $\lambda=\mu=0$ case and then take the $q \rightarrow 1^{-}$limit. In this limit $\left(1-q^{z}\right) /\left(1-q^{\gamma}\right)$ becomes the binomial element $z / \gamma$ and the 
domain of integration becomes $C_{\gamma}^{k_{1}, \ldots, k_{n}}[0,1]$, exactly as in the proof of the $\mathrm{A}_{n}$ Selberg integral (cf. [63, §5]). The resulting $\mathrm{A}_{n}$ Selberg average is the $\ell=k_{1}$ case of Theorem 1.2. It is a trivial exercise to verify that the right-hand side of (1.13) is independent of the choice of $\ell$, as long as $\ell \geqslant l(\lambda)$.

4.3. A companion to the $\mathrm{A}_{n}$ AFLT integral. In 64 the $n=2$ case of Theorem 3.4 with $\mu^{(2)}=0$ is employed to prove an $\mathrm{A}_{2}$ Selberg integral with two Jack polynomials in the integrand, but in a different form to that of the $\mathrm{A}_{2}$ AFLT integral (Theorem 1.2 for $n=2$ ). The two integrals differ in that the argument of the second Jack polynomial in [64, Theorem 3.1] is simply the alphabet $t^{(2)}$ with cardinality $k_{2}$ and there is an additional parameter $\beta_{1}$ subject to $\beta_{1}+\beta_{2}=\gamma+1$ (here $\beta_{2}$ is the $\beta$ of the $\mathrm{A}_{2}$ AFLT integral). By the rank- $n$ case of Theorem 3.4 we obtain an $\mathrm{A}_{n}$ analogue of [64, Theorem 3.1] described below.

For $\alpha_{1}, \ldots, \alpha_{n}, \beta_{n-1}, \beta_{n}, \gamma \in \mathbb{C}$ such that

$$
\begin{gathered}
\beta_{n-1}+\beta_{n}=\gamma+1, \\
\beta_{n-1}+\left(i-k_{n}-1\right) \gamma \notin \mathbb{Z} \text { for } 1 \leqslant i \leqslant \min \left\{k_{n-1}, k_{n}\right\}
\end{gathered}
$$

and

$$
\begin{aligned}
& \operatorname{Re}(\gamma)>-\frac{1}{\max \left\{k_{n-1}, k_{n}\right\}}, \quad \operatorname{Re}\left(\beta_{r}+\left(i-k_{r+1}-1\right) \gamma\right)>0 \quad \text { for } 1 \leqslant r \leqslant n \text { and } 1 \leqslant i \leqslant k_{r} \\
& \operatorname{Re}\left(\alpha_{r}+\cdots+\alpha_{s}+(r-s+i-1) \gamma\right)>0 \\
& \quad \text { for }\left\{\begin{array}{l}
1 \leqslant r \leqslant s \leqslant n-1 \text { and } 1 \leqslant i \leqslant k_{r}-k_{r-1}, \\
1 \leqslant r \leqslant n-1, s=n \text { and } 1 \leqslant i \leqslant \min \left\{k_{n}, k_{r}-k_{r-1}\right\} \\
r=s=n \text { and } 1 \leqslant i \leqslant k_{n}
\end{array}\right.
\end{aligned}
$$

where $k_{n+1}:=0$ and $\beta_{1}=\cdots=\beta_{n-2}:=1$, we modify the $\mathrm{A}_{n}$ Selberg average (1.9) to

$$
\langle\mathscr{O}\rangle_{\alpha_{1}, \ldots, \alpha_{n}, \beta_{n-1}, \beta_{n} ; \gamma}^{k_{1}, \ldots, k_{n}}:=\frac{I_{k_{1}, \ldots, k_{n}}^{\mathrm{A}_{n}}\left(\mathscr{O} ; \alpha_{1}, \ldots, \alpha_{n}, \beta_{n-1}, \beta_{n} ; \gamma\right)}{I_{k_{1}, \ldots, k_{n}}^{\mathrm{A}_{n}}\left(1 ; \alpha_{1}, \ldots, \alpha_{n}, \beta_{n-1}, \beta_{n} ; \gamma\right)} .
$$

Here

$$
\begin{aligned}
& I_{k_{1}, \ldots, k_{n}}^{\mathrm{A}_{n}}\left(\mathscr{O} ; \alpha_{1}, \ldots, \alpha_{n}, \beta_{n-1}, \beta_{n} ; \gamma\right) \\
&:=\int_{C_{\beta_{n-1}, \gamma}^{k_{1}, \ldots, k_{n}[0,1]}} \mathscr{O}\left(t^{(1)}, \ldots, t^{(n)}\right) \prod_{r=1}^{n} \prod_{i=1}^{k_{r}}\left(t_{i}^{(r)}\right)^{\alpha_{r}-1}\left(1-t_{i}^{(r)}\right)^{\beta_{r}-1} \\
& \quad \times \prod_{r=1}^{n}\left|\Delta\left(t^{(r)}\right)\right|^{2 \gamma} \prod_{r=1}^{n-1}\left|\Delta\left(t^{(r)}, t^{(r+1)}\right)\right|^{-\gamma} \mathrm{d} t^{(1)} \cdots \mathrm{d} t^{(n)}
\end{aligned}
$$

and $C_{\beta ; \gamma}^{k_{1}, \ldots, k_{n}}[0,1]$ is the following $\beta$-deformation of the chain defined in Section 4.1, Let

$$
E^{k_{1}, \ldots, k_{n}}[0,1] \subseteq[0,1]^{k_{1}+\cdots+k_{n}}
$$

be the set of points

$$
\left(t^{(1)}, \ldots, t^{(n)}\right) \in[0,1]^{k_{1}+\cdots+k_{n}}
$$


such that

$$
0<t_{1}^{(r)}<\cdots<t_{k_{r}}^{(r)}<1 \quad \text { for } 1 \leqslant r \leqslant n
$$

and

$$
t_{i}^{(r)}<t_{i-k_{r}+k_{r+1}}^{(r+1)} \quad \text { for } 1 \leqslant i \leqslant k_{r}, 1 \leqslant r \leqslant n-2 .
$$

$E^{k_{1}, \ldots, k_{n}}[0,1]$ differs from the set $D^{k_{1}, \ldots, k_{n}}[0,1]$ only in that the relative ordering between the variables $t^{(n-1)}$ and $t^{(n)}$ has been removed. Accordingly we replace the sum over the maps $M_{n-1}$ by a sum over maps

$$
M_{n-1}^{\prime}:\left\{1, \ldots, k_{n}\right\} \longrightarrow\left\{1, \ldots, k_{n+1}+1\right\}
$$

subject to $M_{n-1}^{\prime}(i) \leqslant M_{n-1}^{\prime}(i+1)$ for $1 \leqslant i \leqslant k_{n-1}$ such that

$$
t_{M_{n-1}^{\prime}(i)-1}^{(n)}<t_{i}^{(n-1)}<t_{M_{n-1}^{\prime}(i)}^{(n)}
$$

where $t_{0}^{(n)}:=0$ and $t_{k_{n}+1}^{(n)}:=1$. We then define $E_{M_{1}, \ldots, M_{n-2} ; M_{n-1}^{\prime}}^{k_{1}, \ldots, k_{n}}[0,1] \subseteq E^{k_{1}, \ldots, k_{n}}[0,1]$ by requiring that (4.1) holds for $M_{1}, \ldots, M_{n-2}$ and (4.7) holds for $M_{n-1}$. Hence

$$
E^{k_{1}, \ldots, k_{n}}[0,1]=\sum_{M_{1}, \ldots, M_{n-2}, M_{n-1}^{\prime}} E_{M_{1}, \ldots, M_{n-2} ; M_{n-1}^{\prime}}^{k_{1}, \ldots, k_{n}}[0,1] .
$$

We also replace the weight function (4.2) by

$$
G_{M_{1}, \ldots, M_{n-2}, M_{n-1}^{\prime}}^{k_{1}, \ldots, k_{n}}(\gamma):=F_{M_{1}, \ldots, M_{n-2}}^{k_{1}, \ldots, k_{n-1}}(\gamma) \prod_{i=1}^{k_{n-1}} \frac{\sin \left(\pi\left(\beta-\left(i+k_{n}-k_{n-1}-M_{n-1}^{\prime}(i)+1\right) \gamma\right)\right)}{\sin \left(\pi\left(\beta-\left(i+k_{n}-k_{n-1}\right) \gamma\right)\right)}
$$

Note that the condition (4.5b) is necessary for this weight function to be free of poles. The new chain is then defined as

$$
C_{\beta ; \gamma}^{k_{1}, \ldots, k_{n}}[0,1]:=\sum_{M_{1}, \ldots, M_{n-2}, M_{n-1}^{\prime}} G_{M_{1}, \ldots, M_{n-2}, M_{n-1}^{\prime}}^{k_{1}, \ldots, k_{n}}(\gamma) D_{M_{1}, \ldots, M_{n-1}}^{k_{1}, \ldots, k_{n}}[0,1] .
$$

We are now ready to state the counterpart to Theorem 1.2 ,

Theorem 4.1. For $n \geqslant 2$, let $k_{1}, \ldots, k_{n}$ be nonnegative integers such that $k_{1} \leqslant \cdots \leqslant k_{n-1}$. Then for $\alpha_{1}, \ldots, \alpha_{n}, \beta_{n-1}, \beta_{n}, \gamma \in \mathbb{C}$ such that (4.5) holds and $\lambda, \mu \in \mathscr{P}$, we have

$$
\begin{aligned}
\left\langle P_{\lambda}^{(1 / \gamma)}\right. & \left.\left(t^{(1)}\right) P_{\mu}^{(1 / \gamma)}\left(t^{(n)}\right)\right\rangle_{\alpha_{1}, \ldots, \alpha_{n}, \beta_{n-1}, \beta_{n} ; \gamma}^{k_{1}, \ldots, k_{n}} \\
= & P_{\lambda}^{(1 / \gamma)}\left[k_{1}\right] P_{\mu}^{(1 / \gamma)}\left[k_{n}\right] \prod_{r=1}^{n-1} \frac{\left(\alpha_{1}+\cdots+\alpha_{r}+\left(k_{1}-r\right) \gamma ; \gamma\right)_{\lambda}}{\left(\alpha_{1}+\cdots+\alpha_{r}+\beta_{r}+\left(k_{1}+k_{r}-k_{r+1}-r-1\right) \gamma ; \gamma\right)_{\lambda}} \\
& \times \prod_{r=2}^{n} \frac{\left(\alpha_{r}+\cdots+\alpha_{n}+\left(k_{n}+r-n-1\right) \gamma ; \gamma\right)_{\mu}}{\left(1+\alpha_{r}+\cdots+\alpha_{n}-\beta_{r-1}+\left(k_{r}-k_{r-1}+k_{n}+r-n-1\right) \gamma ; \gamma\right)_{\mu}} \\
& \times \prod_{i=1}^{k_{1}} \prod_{j=1}^{k_{n}} \frac{\left(\alpha_{1}+\cdots+\alpha_{n}+\left(k_{1}+k_{n}-n-i-j+1\right) \gamma\right)_{\lambda_{i}+\mu_{j}}}{\left(\alpha_{1}+\cdots+\alpha_{n}+\left(k_{1}+k_{n}-n-i-j+2\right) \gamma\right)_{\lambda_{i}+\mu_{j}}}
\end{aligned}
$$

and

$$
I_{k_{1}, \ldots, k_{n}}^{\mathrm{A}_{n}}\left(1 ; \alpha_{1}, \ldots, \alpha_{n}, \beta_{n-1}, \beta_{n} ; \gamma\right)
$$




$$
\begin{aligned}
= & \prod_{r=1}^{n} \prod_{i=1}^{k_{r}} \frac{\Gamma\left(\beta_{r}+\left(i-k_{r+1}-1\right) \gamma\right) \Gamma(i \gamma)}{\Gamma(\gamma)} \\
& \times \prod_{1 \leqslant r \leqslant s \leqslant n-1} \prod_{i=1}^{k_{r}-k_{r-1}} \frac{\Gamma\left(\alpha_{r}+\cdots+\alpha_{s}+(r-s+i-1) \gamma\right)}{\Gamma\left(\alpha_{r}+\cdots+\alpha_{s}+\beta_{s}+\left(k_{s}-k_{s+1}+r-s+i-2\right) \gamma\right)} \\
& \times \prod_{r=1}^{n} \prod_{i=1}^{k_{n}} \frac{\Gamma\left(\alpha_{r}+\cdots+\alpha_{n}+(r-n+i-1) \gamma\right)}{\Gamma\left(1+\alpha_{r}+\cdots+\alpha_{n}-\beta_{r-1}+\left(k_{r}-k_{r-1}+r-n+i-1\right) \gamma\right)},
\end{aligned}
$$

where $k_{0}=k_{n+1}:=0$ and $\beta_{0}=\cdots=\beta_{n-2}:=1$.

It should be noted that the final product in (4.9) may alternatively be expressed as

$$
\prod_{r=1}^{n-1} \prod_{i=1}^{k_{r}-k_{r}-1} \frac{\Gamma\left(\alpha_{r}+\cdots+\alpha_{n}+(r-n+i-1) \gamma\right)}{\Gamma\left(\alpha_{r}+\cdots+\alpha_{n}+\left(k_{n}+r-n+i-1\right) \gamma\right)} \prod_{i=1}^{k_{n}} \frac{\Gamma\left(\alpha_{n}+(i-1) \gamma\right)}{\Gamma\left(\alpha_{n}+\beta_{n}+\left(k_{n}-k_{n-1}+i-2\right) \gamma\right)}
$$

When $\beta=\gamma$ in (1.13) and $\left(\beta_{n-1}, \beta_{n}\right)=(1, \gamma)$ in (4.8) both integral evaluations coincide. For $k_{n}=0$ equation (4.8) simplifies to the $\mathrm{A}_{n-1}$ analogue of Kadell's integral [63, Theorem 6.1].

Proof. We start with (3.11) with $k_{n}$ finite and, for $\lambda, \mu \in \mathscr{P}$ with $l(\lambda) \leqslant k_{1}$ and $l(\mu) \leqslant k_{n}$, make the substitutions

$$
\left(X^{(1)}, Y^{(n)}, a_{n-1}, t, \mu^{(n)}\right) \mapsto\left(\langle\lambda\rangle_{k_{1}}, q^{\alpha_{n}}\langle\mu\rangle_{k_{n}}, q^{\beta_{n-1}+\left(k_{n-1}-k_{n}\right) \gamma}, q^{\gamma}, 0\right)
$$

and

$$
z_{r} \mapsto q^{\alpha_{r}-\gamma} \quad \text { for } 1 \leqslant r \leqslant n-1 .
$$

Then the resulting sum may be turned into an integral following the steps outlined in Section 4.2 . For $\lambda=\mu=0$ this yields (4.9) and for general $\lambda$ and $\mu$ it gives

$$
\left\langle P_{\lambda}^{(1 / \gamma)}\left(t^{(1)}\right) P_{\mu}^{(1 / \gamma)}\left(t^{(n)}\right)\right\rangle_{\alpha_{1}, \ldots, \alpha_{n}, \beta_{n-1}, \beta_{n} ; \gamma}^{k_{1}, \ldots, k_{n}} \times I_{k_{1}, \ldots, k_{n}}^{\mathrm{A}_{n}}\left(1 ; \alpha_{1}, \ldots, \alpha_{n}, \beta_{n-1}, \beta_{n} ; \gamma\right) .
$$

\section{Complex Schur functions and Selberg integrals}

In this section we introduce a complex analogue of the Schur function and show how complex Schur functions may be utilised to prove $\mathrm{A}_{n}$ Selberg-type integrals, such as Theorem 1.3. We should remark that Kadell already observed in [25] that for $\gamma=1$ the evaluations of the Kadell and Hua-Kadell integrals remain valid if one replaces the Schur functions in the integrand by Schur functions indexed by sequences of complex numbers. His paper does not, however, provide the necessary tools to attack integrals such as (1.22), and we will not use any of his results for complex Schur functions, such as Pieri and branching rules.

5.1. Complex Schur functions. In the following we fix the principal branch of the complex logarithm with cut along the negative real axis and argument in $(-\pi, \pi]$. Accordingly we denote the cut or slit complex plane $|\operatorname{Im}(\log (x))|<\pi$ by $\Omega$.

For $x=\left(x_{1}, \ldots, x_{n}\right) \in \Omega^{n}$ and $z=\left(z_{1}, \ldots, z_{n}\right) \in \mathbb{C}^{n}$, we define the complex Schur function 6

$$
S^{(n)}(x ; z):=\frac{\operatorname{det}_{1 \leqslant i, j \leqslant n}\left(x_{i}^{z_{j}}\right)}{\Delta(x)},
$$

\footnotetext{
${ }^{6}$ Our definition differs slightly from that of Kadell [25].
} 
where $\Delta(x)$ is the Vandermonde product (1.7). Clearly, for $\lambda \in \mathscr{P}_{n}$,

$$
s_{\lambda}\left(x_{1}, \ldots, x_{n}\right)=S^{(n)}\left(x_{1}, \ldots, x_{n} ; \lambda_{1}+n-1, \lambda_{2}+n-2, \ldots, \lambda_{n}\right) .
$$

By removing the singularities at $x_{i}=x_{j}$ we extend $S^{(n)}$ to a holomorphic function on $\Omega^{n}$. Since the complex Schur function is symmetric in $x$, we may employ the usual plethystic notation and we will sometimes write $S^{(n)}([x] ; z)$ instead of $S^{(n)}(x ; z)$, where of course $x$ should always be an alphabet of cardinality $n$.

It follows immediately from the determinantal structure of $S^{(n)}$ that the following expansion holds.

Lemma 5.1. For any $0 \leqslant m \leqslant n$, we have

$$
\begin{aligned}
S^{(n)}\left(x_{1}, \ldots, x_{n} ; z_{1}, \ldots, z_{n}\right) & =\sum_{\substack{I \subseteq\{1, \ldots, n\} \\
|I|=m}} \frac{S^{(m)}\left(\left[\sum_{i \in I} x_{i}\right] ; z_{1}, \ldots, z_{m}\right) S^{(n-m)}\left(\left[\sum_{i \notin I} x_{i}\right] ; z_{m+1}, \ldots, z_{n}\right)}{\prod_{i \in I} \prod_{j \notin I}\left(x_{i}-x_{j}\right)} .
\end{aligned}
$$

Like the ordinary Schur function, the complex Schur function satisfies a simple specialisation formula.

Lemma 5.2. We have

$$
S^{(n)}(\underbrace{1, \ldots, 1}_{n \text { times }} ; z_{1}, \ldots, z_{n})=S^{(n)}\left([n] ; z_{1}, \ldots, z_{n}\right)=\prod_{1 \leqslant i<j \leqslant n} \frac{z_{i}-z_{j}}{j-i} .
$$

Proof. Since $S^{(n)}\left([n] ; z_{1}, \ldots, z_{n}\right)$ is a polynomial in $z$, the claim follows from the specialisation formula (2.11) and the fact that for arbitrary $\lambda \in \mathscr{P}_{n}$,

$$
S^{(n)}\left([n] ; \lambda_{1}+n-1, \lambda_{2}+n-2, \ldots, \lambda_{n}\right)=s_{\lambda}[n] .
$$

For $0<\theta<\pi$ and $r>0$, let $C_{\theta, r}$ denote the contour in $\mathbb{C}$ going counterclockwise around the border of the angular sector $|x| \leqslant r,|\operatorname{Im}(\log (x))| \leqslant \theta$ as shown below.

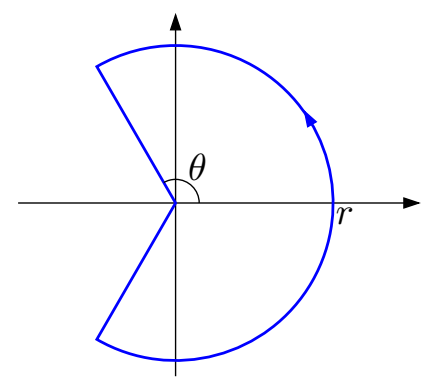

Then the complex Schur function satisfies the following integral identity.

Theorem 5.3. For $\ell$ a nonnegative integer, let $y=\left(y_{1}, \ldots, y_{\ell}\right) \in \Omega^{\ell}$, and let $0<\theta<\pi, r>0$ such that $y_{i} \in \operatorname{int}\left(C_{\theta, r}\right)$ for all $1 \leqslant i \leqslant \ell$. For $k$ a nonnegative integer, let $z=\left(z_{1}, \ldots, z_{k}\right) \in \Omega^{k}$ such that $\operatorname{Re}\left(z_{i}\right)>-1$ for all $1 \leqslant i \leqslant k$. Then, for $\lambda \in \mathscr{P}$,

$$
\frac{1}{k !(2 \pi \mathrm{i})^{k}} \int_{C_{\theta, r}^{k}} S^{(k)}(x ; z) s_{\lambda}[y-x] \prod_{1 \leqslant i<j \leqslant k}\left(x_{i}-x_{j}\right)^{2} \prod_{i=1}^{k} \prod_{j=1}^{\ell}\left(x_{i}-y_{j}\right)^{-1} \mathrm{~d} x_{1} \cdots \mathrm{d} x_{k}
$$




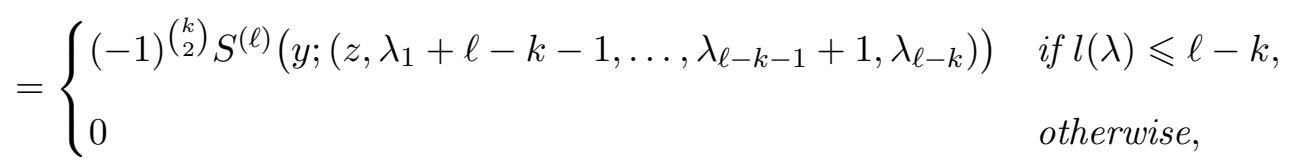

where $C_{\theta, r}^{k}$ denotes the $k$-fold product $C_{\theta, r} \times \cdots \times C_{\theta, r}$.

We repeat our remark from the introduction that the above integral should be understood in the sense of indefinite integrals since the integrand is not defined at $(0, \ldots, 0) \in \mathbb{C}^{k}$.

Proof. The integral in (5.3) is continuous in $y$, so that it suffices to prove the claim on the dense subset of $\operatorname{int}\left(C_{\theta, r}^{k}\right)$ for which the $y_{i}$ are all distinct. In particular, the first three factors of the integrand are holomorphic on $\Omega^{k}$, so that the integrand has only simple poles along the divisors $x_{i}=y_{j}$.

To compute the integral we proceed recursively. For $m$ an integer such that $0 \leqslant m \leqslant k$, define

$$
\begin{aligned}
\mathscr{I}_{m}^{(k, \ell)}(y):= & \frac{(-1)^{\left(\begin{array}{c}
m \\
2
\end{array}\right) m !}}{k !} \sum_{\substack{I \subseteq\{1, \ldots, \ell\} \\
|I|=m}}\left(\prod_{i \in I} \prod_{j \notin I}\left(y_{i}-y_{j}\right)^{-1}\right. \\
& \times \frac{1}{(2 \pi \mathrm{i})^{k-m}} \int_{C_{\theta, r}^{k-m}} S^{(k)}\left(\left[\sum_{i \in I} y_{i}+\sum_{i=m+1}^{k} x_{i}\right] ; z\right) s_{\lambda}\left(\left[\sum_{i \notin I} y_{i}-\sum_{i=m+1}^{k} x_{i}\right]\right) \\
& \left.\times \prod_{m+1 \leqslant i<j \leqslant k}\left(x_{i}-x_{j}\right)^{2} \prod_{i=m+1}^{k} \frac{\prod_{j \in I}\left(x_{i}-y_{j}\right)}{\prod_{j \notin I}\left(x_{i}-y_{j}\right)} \mathrm{d} x_{m+1} \cdots \mathrm{d} x_{k}\right),
\end{aligned}
$$

where we have suppressed the dependence on $z$ and $\lambda$. Observe that $\mathscr{I}_{0}^{(k, \ell)}(y)$ coincides with the left-hand side of (5.3) and that $\mathscr{I}_{m}^{(k, \ell)}(y)=0$ if $m>\ell$.

Now consider $\mathscr{I}_{m}^{(k, \ell)}(y)$ for some fixed $0 \leqslant m \leqslant k-1$. For a given term in the summand indexed by $I$, we compute the integral over $x_{m+1}$ by shrinking the radius $r$ of the corresponding contour $C_{\theta, r}$ to $r_{0}$. Here $r_{0}$ is sufficiently small so that $y_{j} \in \operatorname{ext}\left(C_{\theta, r_{0}}\right)$ for all $j \notin I$. As a result, the integral over $x_{m+1}$ is expressed as a sum over the residues in $x_{m+1}$ at the $\ell-m$ (distinct) poles $x_{m+1}=y_{j}$ for $j \notin I$, plus a remainder $x_{m+1}$-integral over $C_{\theta, r_{0}}$. Since the integrand grows slower than $1 /\left|x_{m+1}\right|$ as $x_{m+1}$ approaches 0 in the angular sector, this remainder converges to 0 as $r_{0} \rightarrow 0$, and hence (since it is independent of $r_{0}$ ) is identically 0 . Thus

$$
\begin{aligned}
\mathscr{I}_{m}^{(k, \ell)}(y)= & \frac{(-1)^{\left(\begin{array}{c}
m+1 \\
2
\end{array}\right)}}{k !} \sum_{\substack{I \subseteq\{1, \ldots, \ell\} \\
|I|=m}} \sum_{r \notin I}\left(\prod_{i \in I \cup\{r\}} \prod_{j \notin I \cup\{r\}}\left(y_{i}-y_{j}\right)^{-1}\right. \\
& \times \frac{1}{(2 \pi \mathrm{i})^{k-m-1}} \int_{C_{\theta, r}^{k-m-1}} S^{(k)}\left(\left[\sum_{i \in I \cup\{r\}} y_{i}+\sum_{i=m+2}^{k} x_{i}\right] ; z\right) s_{\lambda}\left(\left[\sum_{i \notin I \cup\{r\}} y_{i}-\sum_{i=m+2}^{k} x_{i}\right]\right) \\
& \left.\times \prod_{m+2 \leqslant i<j \leqslant k}\left(x_{i}-x_{j}\right)^{2} \prod_{i=m+2}^{k} \frac{\prod_{j \in I \cup\{r\}}\left(x_{i}-y_{j}\right)}{\prod_{j \notin I \backslash\{r\}}\left(x_{i}-y_{j}\right)} \mathrm{d} x_{m+2} \cdots \mathrm{d} x_{k}\right),
\end{aligned}
$$


which of course vanishes for $m \geqslant \ell$. Vanishing or not, defining $J:=I \cup\{r\}$ and noting that there are exactly $m+1$ ways an $(m+1)$-set $J$ can be decomposed into an $m$-set and a singleton, we obtain $\mathscr{I}_{m}^{(k, \ell)}(y)=\mathscr{I}_{m+1}^{(k, \ell)}(y)$. By iteration this yields $\mathscr{I}_{0}^{(k, \ell)}(y)=\mathscr{I}_{k}^{(k, \ell)}(y)$, so that

$$
\mathscr{I}_{0}^{(k, \ell)}(y)=(-1)^{\left(\begin{array}{c}
k \\
2
\end{array}\right)} \sum_{\substack{I \subseteq\{1, \ldots, \ell\} \\
|I|=k}} \frac{S^{(k)}\left(\left[\sum_{i \in I} y_{i}\right] ; z\right) s_{\lambda}\left(\left[\sum_{i \notin I} y_{i}\right]\right)}{\prod_{i \in I} \prod_{j \notin I}\left(y_{i}-y_{j}\right)} .
$$

If $l(\lambda)>\ell-k$ this vanishes and if $l(\lambda) \leqslant \ell-k$ it follows from (5.1) and Lemma 5.1 that

$$
\mathscr{I}_{0}^{(k, \ell)}(y)=(-1)^{\left(\begin{array}{c}
k \\
2
\end{array}\right)} S^{(\ell)}\left(y ;\left(z, \lambda_{1}+\ell-k-1, \ldots, \lambda_{\ell-k-1}+1, \lambda_{\ell-k}\right)\right) .
$$

To prove Theorem 1.3 we not only need Theorem 5.3 but also a generalisation of the $y=$ $(1, \ldots, 1) \in \mathbb{C}^{\ell}$ (or, plethystically, $y=\ell$ ) case of this theorem. For this we require a variant of the classical beta integral.

Lemma 5.4. Let $\alpha, \beta \in \mathbb{C}$ such that $\operatorname{Re}(\alpha)>0$. Then, for $r>1$ and $0<\theta<\pi$,

$$
\frac{1}{2 \pi \mathrm{i}} \int_{C_{\theta, r}} x^{\alpha-1}(x-1)^{\beta-1} \mathrm{~d} x=\frac{\Gamma(\alpha)}{\Gamma(1-\beta) \Gamma(\alpha+\beta)} .
$$

Proof. By holomorphicity, we may assume without loss of generality that $\operatorname{Re}(\beta)>0$ in the following.

Recall that $r>1$, so that the branch point $x=1$ is contained in the interior of $C_{\theta, r}$. Deforming this contour to $\bar{C}_{\theta, r}$ given by

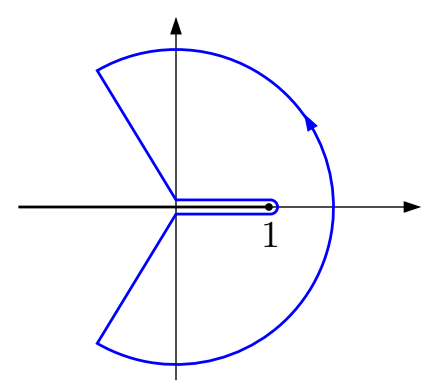

we get, by slight abuse of notation,

$$
\begin{aligned}
\int_{C_{\theta, r}} x^{\alpha-1}(x-1)^{\beta-1} \mathrm{~d} x= & \left(\int_{\bar{C}_{\theta, r}}-\lim _{r_{0} \rightarrow 0} \int_{C_{r_{0}}}\right) x^{\alpha-1}(x-1)^{\beta-1} \mathrm{~d} x \\
& -\left(\mathrm{e}^{\pi \mathrm{i}(\beta-1)} \int_{0}^{1}+\mathrm{e}^{-\pi \mathrm{i}(\beta-1)} \int_{0}^{1}\right) x^{\alpha-1}|x-1|^{\beta-1} \mathrm{~d} x,
\end{aligned}
$$

where $C_{r_{0}}$ is the positively oriented semicircle $1+r_{0} \mathrm{e}^{\mathrm{i} \phi}, \phi \in(-\pi / 2, \pi / 2)$ of radius $r_{0}$ around 1 . The integral over the deformed contour $\bar{C}_{\theta, r}$ trivially vanishes. Since $\operatorname{Re}(\beta)>0$, so does the integral over $C_{r_{0}}$ in the $r_{0} \rightarrow 0$ limit. By the standard Euler beta integral [4, p. 5]

$$
\int_{0}^{1} x^{\alpha-1}(1-x)^{\beta-1} \mathrm{~d} x=\frac{\Gamma(\alpha) \Gamma(\beta)}{\Gamma(\alpha+\beta)}
$$


for $\operatorname{Re}(\alpha), \operatorname{Re}(\beta)>0$, we thus find

$$
\frac{1}{2 \pi \mathrm{i}} \int_{C_{\theta, r}} x^{\alpha-1}(x-1)^{\beta-1} \mathrm{~d} x=\frac{\sin (\pi \beta)}{\pi} \frac{\Gamma(\alpha) \Gamma(\beta)}{\Gamma(\alpha+\beta)} .
$$

The claim now follows by the reflection formula for the gamma function.

Theorem 5.5. Let $\beta \in \mathbb{C}$ and $z=\left(z_{1}, \ldots, z_{k}\right) \in \mathbb{C}^{k}$ such that $\operatorname{Re}\left(z_{i}\right)>-1$ for all $i$. Then, for $r>1,0<\theta<\pi$ and $\lambda \in \mathscr{P}$,

$$
\begin{aligned}
& \frac{1}{(2 \pi \mathrm{i})^{k}} \int_{C_{\theta, r}^{k}} S^{(k)}(x ; z) s_{\lambda}[1-\beta-x] \prod_{1 \leqslant i<j \leqslant k}\left(x_{i}-x_{j}\right)^{2} \prod_{i=1}^{k}\left(x_{i}-1\right)^{\beta-1} \mathrm{~d} x_{1} \cdots \mathrm{d} x_{k} \\
& =(-1)^{\left(\begin{array}{l}
k \\
2
\end{array}\right)} S^{(k)}([k] ; z) s_{\lambda}[1-\beta-k] \\
& \times \prod_{i=1}^{k}\left(\frac{i ! \Gamma\left(z_{i}+1\right)}{\Gamma(2-i-\beta) \Gamma\left(z_{i}+\beta+k\right)} \prod_{j \geqslant 1} \frac{z_{i}-\lambda_{j}+\beta+k+j-1}{z_{i}+\beta+k+j-1}\right) .
\end{aligned}
$$

Proof. First assume that $\beta=1-\ell$ with $\ell$ an integer. Then (5.4) simplifies to

$$
\begin{aligned}
& \frac{1}{(2 \pi \mathrm{i})^{k}} \int_{C_{\theta, r}^{k}} S^{(k)}(x ; z) s_{\lambda}[\ell-x] \prod_{1 \leqslant i<j \leqslant k}\left(x_{i}-x_{j}\right)^{2} \prod_{i=1}^{k}\left(x_{i}-1\right)^{-\ell} \mathrm{d} x_{1} \cdots \mathrm{d} x_{k} \\
& =(-1)^{\left(\begin{array}{c}
k \\
2
\end{array}\right)} S^{(k)}([k] ; z) s_{\lambda}[\ell-k] \\
& \times \prod_{i=1}^{k}\left(\frac{i ! \Gamma\left(z_{i}+1\right)}{\Gamma(\ell-i+1) \Gamma\left(z_{i}-\ell+k+1\right)} \prod_{j \geqslant 1} \frac{z_{i}-\lambda_{j}-\ell+k+j}{z_{i}-\ell+k+j}\right) \\
& =k !(-1)^{\left(\begin{array}{c}
k \\
2
\end{array}\right)} S^{(k)}([k] ; z) s_{\lambda}[\ell-k] \prod_{i=1}^{k} \prod_{j=1}^{\ell-k} \frac{z_{i}-\lambda_{j}-\ell+k+j}{k+j-i} .
\end{aligned}
$$

By the principal specialisation formulas (2.11) and (5.2) it follows that

$$
\begin{aligned}
S^{(k)}([k] ; z) s_{\lambda}[\ell-k] & \prod_{i=1}^{k} \prod_{j=1}^{\ell-k} \frac{z_{i}-\lambda_{j}-\ell+k+j}{k+j-i} \\
& = \begin{cases}S^{(\ell)}\left([\ell] ;\left(z, \lambda_{1}+\ell-k-1, \ldots, \lambda_{\ell-k-1}+1, \lambda_{\ell-k}\right)\right) & \text { if } l(\lambda) \leqslant \ell-k, \\
0 & \text { otherwise, }\end{cases}
\end{aligned}
$$

so that (5.5) is precisely (5.3) for $y=1+\cdots+1=\ell$.

One possible approach to showing the general complex $\beta$ case would be to appeal to Carlson's theorem. To avoid having to show the required bounded growth at infinity of the left-hand side of (5.4), we will more simply show that after appropriate normalisation both sides of (5.4) become rational functions. As a first step towards proving this we make the substitution $\lambda \mapsto \lambda^{\prime}$ using 
(1.20) and

$$
\prod_{j \geqslant 1} \frac{w-\lambda_{j}^{\prime}+j-1}{w+j-1}=\prod_{j \geqslant 1} \frac{w-j}{w+\lambda_{j}-j}
$$

to obtain the equivalent identity

$$
\begin{aligned}
& \frac{1}{(2 \pi \mathrm{i})^{k}} \int_{C_{\theta, r}^{k}} S^{(k)}(x ; z) s_{\lambda}[x+\beta-1] \prod_{1 \leqslant i<j \leqslant k}\left(x_{i}-x_{j}\right)^{2} \prod_{i=1}^{k}\left(x_{i}-1\right)^{\beta-1} \mathrm{~d} x_{1} \cdots \mathrm{d} x_{k} \\
& =(-1)^{\left(\begin{array}{l}
k \\
2
\end{array}\right)} S^{(k)}([k] ; z) s_{\lambda}[\beta+k-1] \\
& \times \prod_{i=1}^{k} \frac{i ! \Gamma\left(z_{i}+1\right)}{\Gamma(2-i-\beta) \Gamma\left(z_{i}+\beta+k\right)} \prod_{i=1}^{k} \prod_{j \geqslant 1} \frac{z_{i}+\beta+k-j}{z_{i}+\lambda_{j}+\beta+k-j} .
\end{aligned}
$$

By [32, p. 72]

$$
s_{\lambda}[X+Y]=\sum_{\mu} s_{\lambda / \mu}[X] s_{\mu}[Y]
$$

and the determinantal nature of both types of Schur functions in the integrand, the left hand side of (5.7) may also be written as

$$
\begin{aligned}
& k ! \sum_{\mu} s_{\lambda / \mu}[\beta-1] \frac{1}{(2 \pi \mathrm{i})^{k}} \int_{C_{\theta, r}^{k}} \operatorname{det}_{1 \leqslant i, j \leqslant k}\left(x_{i}^{\mu_{j}+k-j}\right) \prod_{i=1}^{k} x_{i}^{z_{i}}\left(x_{i}-1\right)^{\beta-1} \mathrm{~d} x_{1} \cdots \mathrm{d} x_{k} \\
& =k ! \sum_{\mu} s_{\lambda / \mu}[\beta-1] \operatorname{det}_{1 \leqslant i, j \leqslant k}\left(\frac{1}{2 \pi \mathrm{i}} \int_{C_{\theta, r}} x^{z_{i}+\mu_{j}+k-j}(x-1)^{\beta-1} \mathrm{~d} x\right) \\
& =k ! \sum_{\mu} s_{\lambda / \mu}[\beta-1] \operatorname{det}_{1 \leqslant i, j \leqslant k}\left(\frac{\Gamma\left(z_{i}+\mu_{j}+k-j+1\right)}{\Gamma(1-\beta) \Gamma\left(z_{i}+\mu_{j}+\beta+k-j+1\right)}\right) \\
& =k ! \sum_{\mu} s_{\lambda / \mu}[\beta-1] \prod_{i=1}^{k} \frac{\Gamma\left(z_{i}+1\right)}{\Gamma(1-\beta) \Gamma\left(z_{i}+\beta+1\right)} \operatorname{det}_{1 \leqslant i, j \leqslant k}\left(\frac{\left(z_{i}+1\right)_{\mu_{j}+k-j}}{\left(z_{i}+\beta+1\right)_{\mu_{j}+k-j}}\right) \text {, }
\end{aligned}
$$

where the penultimate line follows from Lemma [5.4. This shows that the left-hand side of (5.7) divided by the right-hand side is given by the following rational function:

$$
\begin{aligned}
& \frac{(-1)^{\left(\begin{array}{c}
k \\
2
\end{array}\right)}}{S^{(k)}([k] ; z)} \sum_{\mu} \frac{s_{\lambda / \mu}[\beta-1]}{s_{\lambda}[\beta+k-1]} \prod_{i=1}^{k}\left(\frac{\left(z_{i}+\beta+1\right)_{k-1}}{(i-1) !(2-i-\beta)_{i-1}} \prod_{j \geqslant 1} \frac{z_{i}+\lambda_{j}+\beta+k-j}{z_{i}+\beta+k-j}\right) \\
& \times \operatorname{det}_{1 \leqslant i, j \leqslant k}\left(\frac{\left(z_{i}+1\right)_{\mu_{j}+k-j}}{\left(z_{i}+\beta+1\right)_{\mu_{j}+k-j}}\right),
\end{aligned}
$$

completing the proof. We remark that for $\lambda=0$ the above collapses into

$$
\frac{(-1)^{\left(\begin{array}{c}
k \\
2
\end{array}\right)}}{S^{(k)}([k] ; z)} \prod_{i=1}^{k} \frac{\left(z_{i}+\beta+1\right)_{k-1}}{(i-1) !(2-i-\beta)_{i-1}} \operatorname{det}_{1 \leqslant i, j \leqslant k}\left(\frac{\left(z_{i}+1\right)_{k-j}}{\left(z_{i}+\beta+1\right)_{k-j}}\right) \text {. }
$$


In this instance the determinant follows as a special case of a well-known determinant evaluation due to Krattenthaler, see e.g., [28, Lemma 3] with $\left(n, X_{i}, A_{i}, B_{i}\right) \mapsto\left(k, z_{i}, k-i+1, \beta+k-i+1\right)$. For general $\lambda$ it does not seem so easy to show by direct means that the above rational function is in fact equal to 1 .

5.2. Proof of Theorem 1.3. Recall definitions (1.21) of $A_{r}$ and $A_{r, s}$, (1.17) of the integral

$$
I_{k_{1}, \ldots, k_{n}}^{\mathrm{A}_{n}}\left(\mathscr{O} ; \alpha_{1}, \ldots, \alpha_{n}, \beta\right)
$$

and (1.15) of the integration contour $C^{k_{1}, \ldots, k_{n}}$. In our next theorem, which may be viewed as a complex analogue of Theorem 1.3. we will slightly extend the definition of the above integral to allow for functions $\mathscr{O}\left(t^{(1)}, \ldots, t^{(n)}\right)$ that are not polynomial in $t^{(1)}$.

Theorem 5.6. For $n$ a positive integer, let $k_{1}, \ldots, k_{n}$ be nonnegative integers, $\alpha_{1}, \ldots, \alpha_{n}, \beta \in \mathbb{C}$, $z \in \mathbb{C}^{k_{1}}$ and $\lambda^{(2)}, \ldots, \lambda^{(n+1)} \in \mathscr{P}$, such that

$$
\begin{aligned}
\operatorname{Re}\left(z_{i}+\alpha_{1}+\cdots+\alpha_{s}\right)>s-1 & \text { for } 1 \leqslant s \leqslant n \text { and } 1 \leqslant i \leqslant k_{1}, \\
\operatorname{Re}\left(\lambda_{k_{r}-k_{r-1}}^{(r)}+\alpha_{r}+\cdots+\alpha_{s}\right)>s-r & \text { for } 2 \leqslant r \leqslant s \leqslant n .
\end{aligned}
$$

Further let $t^{(1)}, \ldots, t^{(n)}$ be alphabets of cardinality $k_{1}, \ldots, k_{n}$, and set $\lambda_{i}^{(1)}:=z_{i}-k_{1}+i$ for $1 \leqslant i \leqslant k_{1}$ and $t^{(n+1)}:=1-\beta$. Then,

$$
\begin{aligned}
I_{k_{1}, \ldots, k_{n}}^{\mathrm{A}_{n}} & \left(S^{\left(k_{1}\right)}\left(t^{(1)} ; z\right) \prod_{r=2}^{n+1} s_{\lambda^{(r)}}\left[t^{(r)}-t^{(r-1)}\right] ; \alpha_{1}, \ldots, \alpha_{n}, \beta\right) \\
= & S^{\left(k_{1}\right)}\left(\left[k_{1}\right] ; z\right) \prod_{r=1}^{n}\left((-1)^{\left(\begin{array}{c}
k_{r} \\
2
\end{array}\right)} s_{\lambda^{(r+1)}}\left[k_{r+1}-k_{r}\right] \prod_{i=1}^{k_{r}} \frac{i !}{\Gamma\left(k_{r+1}-i+1\right)}\right) \\
& \times \prod_{1 \leqslant r<s \leqslant n} \prod_{i=1}^{k_{r}-k_{r-1}} \prod_{j=1}^{k_{s}-k_{s-1}}\left(\lambda_{i}^{(r)}-\lambda_{j}^{(s)}+A_{r, s}+j-i\right) \\
& \times \prod_{r=1}^{n} \prod_{i=1}^{k_{r}-k_{r-1}}\left(\frac{\Gamma\left(\lambda_{i}^{(r)}+A_{r}-i-n\right)}{\Gamma\left(\lambda_{i}^{(r)}+A_{r, n+1}-i+1\right)} \prod_{j \geqslant 1} \frac{\lambda_{i}^{(r)}-\lambda_{j}^{(n+1)}+A_{r, n+1}+j-i}{\lambda_{i}^{(r)}+A_{r, n+1}+j-i}\right)
\end{aligned}
$$

where $\beta_{1}=\cdots=\beta_{n-1}:=1, \beta_{n}:=\beta, k_{0}:=0$ and $k_{n+1}:=1-\beta$.

We note that

$$
\prod_{r=1}^{n} \prod_{i=1}^{k_{r}} \frac{1}{\Gamma\left(k_{r+1}-i+1\right)}
$$

vanishes unless $k_{r+1}-k_{r} \geqslant 0$ for $1 \leqslant r \leqslant n-1$, i.e., unless $k_{1} \leqslant k_{2} \leqslant \cdots \leqslant k_{n}$. Assuming this holds it then follows from

$$
\prod_{r=1}^{n} s_{\lambda^{(r+1)}}\left[k_{r+1}-k_{r}\right]
$$

that the right-hand side vanishes unless $l\left(\lambda^{(r)}\right) \leqslant k_{r}-k_{r-1}$ for all $2 \leqslant r \leqslant n$.

If we take $\lambda^{(2)}=\lambda^{(n+1)}=0$ in Theorem 5.6 and also choose $z_{i}=k_{1}-i$ for all $1 \leqslant i \leqslant k_{1}$ (so that on the right $\lambda^{(1)}=0$ ), we obtain

$$
I_{k_{1}, \ldots, k_{n}}^{\mathrm{A}_{n}}\left(1 ; \alpha_{1}, \ldots, \alpha_{n}, \beta\right)
$$




$$
=\prod_{r=1}^{n}\left((-1)^{\left(\begin{array}{c}
k_{r} \\
2
\end{array}\right)} \prod_{i=1}^{k_{r}} \frac{i !}{\Gamma\left(k_{r+1}-i+1\right)}\right) \prod_{1 \leqslant r<s \leqslant n+1} \prod_{i=1}^{k_{r}-k_{r-1}} \frac{\Gamma\left(A_{r, s}+k_{s}-k_{s-1}-i+1\right)}{\Gamma\left(A_{r, s}-i+1\right)},
$$

where the $\alpha_{r}$ must satisfy (1.16). This is equivalent to (1.18) where it should be noted that in the above $k_{n+1}$ is defined as $1-\beta$ whereas $k_{n+1}:=0$ in (1.18).

Let $\lambda^{(1)} \in \mathscr{P}_{k_{1}}$. If we take (5.8) for arbitrary partitions $\lambda^{(2)}, \ldots, \lambda^{(n+1)}$, specialise $z_{i}=\lambda_{i}^{(1)}+k_{1}-i$ for $1 \leqslant i \leqslant k_{1}$ and divide the resulting identity by (5.9), then Theorem 1.3 follows with $\ell_{r}$ given by $k_{r}-k_{r-1}$ for all $1 \leqslant r \leqslant n$ and $\ell_{n+1}$ any integer such that $\ell_{n+1} \geqslant l\left(\lambda^{(n+1)}\right)$. Since the right-hand side of (1.22) is independent of the choice of $\ell_{r}$ provided that $\ell_{r} \geqslant l\left(\lambda^{(r)}\right)$, this completes the proof of Theorem 1.3 .

Proof of Theorem 5.6. We may deform the contour $C^{k_{1}, \ldots, k_{n}}$ to

$$
C_{\theta_{1}, r_{1}}^{k_{1}} \times \cdots \times C_{\theta_{n}, r_{n}}^{k_{n}},
$$

for arbitrary real $r_{1}, \ldots, r_{n}$ and $\theta_{1}, \ldots, \theta_{n}$ such that $r_{1}>\cdots>r_{n}>1$ and $\pi>\theta_{1}>\cdots>\theta_{n}>0$, and in the following we assume this new deformed contour.

We now proceed by induction on $n$. The base case, $n=1$, corresponds to Theorem 5.5 with $(k, \lambda, \beta) \mapsto\left(k_{1}, \lambda^{(2)}, \beta_{1}=\beta\right)$ and $z_{i} \mapsto z_{i}+\alpha_{1}-1$ for all $1 \leqslant i \leqslant k_{1}$.

To carry out the inductive step we denote the left-hand side of (5.8) by

$$
\mathscr{L}_{\lambda^{(2)}, \ldots, \lambda^{(n+1)}}^{k_{1}, \ldots, k_{n}}\left(z ; \alpha_{1}, \ldots, \alpha_{n}, \beta\right) .
$$

Assuming $n \geqslant 2$, we integrate over $t^{(1)}$ using Theorem 5.3 with $(k, \ell, x, y, \lambda) \mapsto\left(k_{1}, k_{2}, t^{(1)}, t^{(2)}, \lambda^{(2)}\right)$ and $z_{i} \mapsto z_{i}+\alpha_{1}-1$ for all $1 \leqslant i \leqslant k_{1}$. This yields

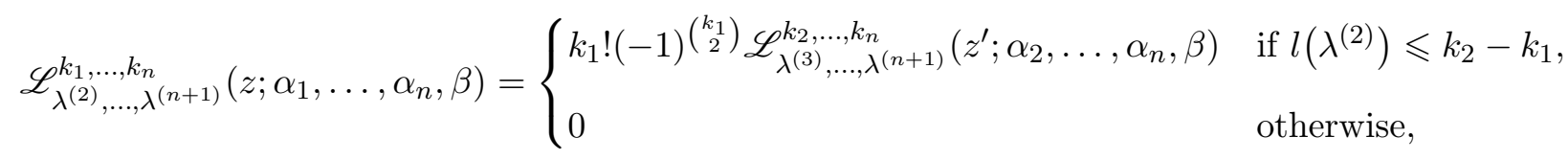

where

$$
z^{\prime}:=\left(z_{1}+\alpha_{1}-1, \ldots, z_{k_{1}}+\alpha_{1}-1, \lambda_{1}^{(2)}+k_{2}-k_{1}-1, \ldots, \lambda_{k_{2}-k_{1}-1}^{(2)}+1, \lambda_{k_{2}-k_{1}}^{(2)}\right) .
$$

By induction on $n$ this gives (in the $l\left(\lambda^{(2)}\right) \leqslant k_{2}-k_{1}$ case)

$$
\begin{aligned}
& \mathscr{L}_{\lambda^{(2)}, \ldots, \lambda(n+1)}^{k_{1}, \ldots, k_{n}}\left(z ; \alpha_{1}, \ldots, \alpha_{n}, \beta\right) \\
& =S^{\left(k_{2}\right)}\left(\left[k_{2}\right] ; z^{\prime}\right) k_{1} !(-1)^{\left(\begin{array}{c}
k_{1} \\
2
\end{array}\right)} \prod_{r=2}^{n}\left(k_{r} !(-1)^{\left(\begin{array}{c}
k_{r} \\
2
\end{array}\right)} s_{\lambda^{(r+1)}}\left[k_{r+1}-k_{r}\right] \prod_{i=1}^{k_{r}} \frac{i !}{\Gamma\left(k_{r+1}-i+1\right)}\right) \\
& \times \prod_{s=3}^{n} \prod_{i=1}^{k_{2}} \prod_{j=1}^{k_{s}-k_{s-1}}\left(z_{i}^{\prime}-\lambda_{j}^{(s)}+\alpha_{2}+\cdots+\alpha_{s-1}+k_{s-1}-k_{s}-s+j+2\right) \\
& \times \prod_{3 \leqslant r<s \leqslant n} \prod_{i=1}^{k_{r}-k_{r}-1} \prod_{j=1}^{k_{s}-k_{s}-1}\left(\lambda_{i}^{(r)}-\lambda_{j}^{(s)}+A_{r, s}+j-i\right) \\
& \times \prod_{i=1}^{k_{2}}\left(\frac{\Gamma\left(z_{i}^{\prime}+\alpha_{2}+\cdots+\alpha_{n}-n+2\right)}{\Gamma\left(z_{i}^{\prime}+\alpha_{2}+\cdots+\alpha_{n}+k_{n}-k_{n+1}-n+2\right)}\right.
\end{aligned}
$$




$$
\begin{gathered}
\left.\times \prod_{j \geqslant 1} \frac{z_{i}^{\prime}-\lambda_{j}^{(n+1)}+\alpha_{2}+\cdots+\alpha_{n}+k_{n}-k_{n+1}+j-n+1}{z_{i}^{\prime}+\alpha_{2}+\cdots+\alpha_{n}+k_{n}-k_{n+1}+j-n+1}\right) \\
\times \prod_{r=3}^{n} \prod_{i=1}^{k_{r}-k_{r-1}}\left(\frac{\Gamma\left(\lambda_{i}^{(r)}+A_{r}-i-n\right)}{\Gamma\left(\lambda_{i}^{(r)}+A_{r, n+1}-i+1\right)} \prod_{j \geqslant 1} \frac{\lambda_{i}^{(r)}-\lambda_{j}^{(n+1)}+A_{r, n+1}+j-i}{\lambda_{i}^{(r)}+A_{r, n+1}+j-i}\right) .
\end{gathered}
$$

By the definition of $z^{\prime}$ and by (5.6) with $(\ell, k, z, \lambda) \mapsto\left(k_{2}, k_{1}, z+\alpha_{1}-1, \lambda^{(2)}\right)$, this finally yields

$$
\begin{aligned}
& \mathscr{L}_{\lambda^{(2)}, \ldots, \lambda^{(n+1)}}^{k_{1}, \ldots, k_{n}}\left(z ; \alpha_{1}, \ldots, \alpha_{n}, \beta\right) \\
& =S^{\left(k_{1}\right)}\left(\left[k_{1}\right] ; z\right) \prod_{r=1}^{n}\left((-1)^{\left(\begin{array}{c}
k_{r} \\
2
\end{array}\right)} s_{\lambda^{(r+1)}}\left[k_{r+1}-k_{r}\right] \prod_{i=1}^{k_{r}} \frac{i !}{\Gamma\left(k_{r+1}-i+1\right)}\right) \\
& \times \prod_{1 \leqslant r<s \leqslant n} \prod_{i=1}^{k_{r}-k_{r-1}} \prod_{j=1}^{k_{s}-k_{s-1}}\left(\lambda_{i}^{(r)}-\lambda_{j}^{(s)}+A_{r, s}+j-i\right) \\
& \times \prod_{r=1}^{n} \prod_{i=1}^{k_{r}-k_{r-1}}\left(\frac{\Gamma\left(\lambda_{i}^{(r)}+A_{r}+r-i-n\right)}{\Gamma\left(\lambda_{i}^{(r)}+A_{r, n+1}+r-i+1\right)} \prod_{j \geqslant 1} \frac{\lambda_{i}^{(r)}-\lambda_{j}^{(n+1)}+A_{r, n+1}+j-i}{\lambda_{i}^{(r)}+A_{r, n+1}+j-i}\right) .
\end{aligned}
$$

As noted previously, the right-hand side vanishes if $k_{2}-k_{1}<0$, and assuming $k_{2}-k_{1} \geqslant 0$ it then vanishes unless $l\left(\lambda^{(2)}\right) \leqslant k_{2}-k_{1}$. Hence the above holds regardless of the ordering of $k_{1}$ and $k_{2}$ or the length of $\lambda^{(2)}$.

\section{An ELliptic AFLT integral}

In this section we prove the elliptic AFLT integral of Theorem 1.4. Throughout it is assumed that $p, q \in \mathbb{C}$ such that $|p|,|q|<1$.

6.1. The elliptic Selberg integral. We begin with a brief review of the elliptic Selberg integral and its relation to the ordinary Selberg integral (1.4).

The elliptic Selberg integral7 was first conjectured by van Diejen and Spiridonov [9, 10] and then proved by the second author in [44]. (See also [21, 54] for alternative proofs). It corresponds to the $\boldsymbol{\lambda}=\boldsymbol{\mu}=\mathbf{0}$ case of Theorem 1.4, viz.

$$
\begin{aligned}
& S_{n}\left(t_{1}, \ldots, t_{6} ; t ; p, q\right) \\
& \quad:=\kappa_{n} \int_{\mathbb{T}^{n}} \prod_{1 \leqslant i<j \leqslant n} \frac{\Gamma\left(t z_{i}^{ \pm} z_{j}^{ \pm} ; p, q\right)}{\Gamma\left(z_{i}^{ \pm} z_{j}^{ \pm} ; p, q\right)} \prod_{i=1}^{n} \frac{\prod_{r=1}^{6} \Gamma\left(t_{r} z_{i}^{ \pm} ; p, q\right)}{\Gamma\left(z_{i}^{ \pm 2} ; p, q\right)} \frac{\mathrm{d} z_{1}}{z_{1}} \cdots \frac{\mathrm{d} z_{n}}{z_{n}} \\
& \quad=\prod_{i=1}^{n}\left(\Gamma\left(t^{i} ; p, q\right) \prod_{1 \leqslant r<s \leqslant 6} \Gamma\left(t^{i-1} t_{r} t_{s} ; p, q\right)\right),
\end{aligned}
$$

where $t, t_{1}, \ldots, t_{6} \in \mathbb{C}$ are such that $|t|,\left|t_{1}\right|, \ldots,\left|t_{6}\right|<1$ and the balancing condition (1.25) holds. For $n=1$ the elliptic Selberg integral corresponds to Spiridonov's elliptic analogue of the Euler

\footnotetext{
${ }^{7}$ An elliptic Selberg integral of a very different type, which arises as a solution of the Knizhnik-ZamolodchikovBernard heat equation, may be found in [57] and references therein.
} 
beta integral [53] and for general $n$ it was shown in 43 . that by taking an appropriate limit the full Selberg integral (1.4) (with $k \mapsto n)$ arises. Specifically, setting $q=\exp (2 \pi \mathrm{i} \tau)$ for $\tau \in \mathbb{H}$

$$
\begin{aligned}
\lim _{p \rightarrow 0} \lim _{\tau \rightarrow 0} \prod_{i=1}^{n} \frac{\Gamma\left(q^{\alpha+\beta+(2 n-i-1) \gamma} ; p, q\right)}{\Gamma\left(q^{i \gamma} ; p, q\right) \Gamma\left(q^{\alpha+(i-1) \gamma} ; p, q\right) \Gamma\left(q^{\beta+(i-1) \gamma} ; p, q\right) \Gamma^{4}\left(-q^{(\alpha+\beta) / 2+(i-1) \gamma} ; p, q\right)} \\
\times S_{n}\left(q^{\alpha / 2}, q^{\alpha / 2},-q^{\beta / 2},-q^{\beta / 2}, p^{1 / 2} q^{1 / 2-\zeta / 2}, p^{1 / 2} q^{1 / 2-\zeta / 2} ; q^{\gamma} ; p, q\right) \\
=\prod_{i=1}^{n} \frac{\Gamma(\alpha+\beta+(2 n-i-1) \gamma) \Gamma(1+\gamma)}{\Gamma(\alpha+(i-1) \gamma) \Gamma(\beta+(i-1) \gamma) \Gamma(1+i \gamma)} \\
\quad \times \int_{[0,1]^{n}} \prod_{i=1}^{n} t_{i}^{\alpha-1}\left(1-t_{i}\right)^{\beta-1} \prod_{1 \leqslant i<j \leqslant n}\left|t_{i}-t_{j}\right|^{2 \gamma} \mathrm{d} t_{1} \cdots \mathrm{d} t_{n},
\end{aligned}
$$

where $\operatorname{Re}(\alpha), \operatorname{Re}(\beta), \operatorname{Re}(\gamma)>0,2(n-1) \gamma+\alpha+\beta-\zeta=0$, see [43, Theorem 7.4]. This same limit, taken on the right-hand side of (6.1) yields 1, so that the Selberg integral follows, albeit for a slightly more restricted range of the parameter $\gamma$ than strictly necessary.

6.2. Elliptic interpolation functions. To lift the elliptic Selberg integral (6.1) to the elliptic AFLT integral of Theorem 1.4 we require a number of results from the theory of elliptic interpolation functions on root systems. For a detailed discussion of these functions we refer the reader to [8, 42, 44, 45, 48, and below we only state some of the key results needed in the proof of Theorem 1.4.

In order to describe the elliptic skew interpolation functions we need the $\mathrm{BC}_{n}$-symmetric elliptic interpolation functions (or well-poised Macdonald functions)

$$
R_{\mu}^{*}\left(x_{1}, \ldots, x_{n} ; a, b ; q, t ; p\right),
$$

where $\mu \in \mathscr{P}_{n}$. For $\lambda \in \mathscr{P}_{n}$ such that $\mu \nsubseteq \lambda$ these functions satisfy the vanishing property

$$
R_{\mu}^{*}\left(a\langle\lambda\rangle_{n ; q, t} ; a, b ; q, t ; p\right)=0 .
$$

The elliptic interpolation function $R_{\mu}^{*}(a, b ; q, t ; p)$ generalises Okounkov's $\mathrm{BC}_{n}$-symmetric Macdonald interpolation polynomial $P_{\mu}^{*}(q, t, s)$ [40] which has the same vanishing property and has the ordinary Macdonald polynomial $P_{\mu}(q, t)$ as top-homogeneous degree component. When $t^{n} a b=p q$, the elliptic interpolation functions are referred to as being of Cauchy-type and factorise as

$$
R_{\mu}^{*}\left(x_{1}, \ldots, x_{n} ; a, b ; q, t ; p\right)=\Delta_{\mu}^{0}\left(t^{n-1} a / b \mid t^{n-1} a x_{1}^{ \pm}, \ldots, t^{n-1} a x_{n}^{ \pm} ; q, t ; p\right),
$$

where $\Delta_{\mu}^{0}\left(a \mid \ldots, x_{i}^{ \pm}, \ldots ; q, t ; p\right):=\Delta_{\mu}^{0}\left(a \mid \ldots, x_{i}, x_{i}^{-1}, \ldots ; q, t ; p\right)$. They also factor under principal specialisation:

$$
R_{\mu}^{*}\left(v\langle 0\rangle_{n ; q, t} ; a, b ; q, t ; p\right)=\Delta_{\mu}^{0}\left(t^{n-1} a / b \mid t^{n-1} a v, a / v ; q, t ; p\right),
$$

and satisfy the evaluation symmetry

$$
\frac{R_{\mu}^{*}\left(v\langle\lambda\rangle_{n ; q, t} / a ; a, b / a ; q, t ; p\right)}{R_{\mu}^{*}\left(v\langle 0\rangle_{n ; q, t} / a ; a, b / a ; q, t ; p\right)}=\frac{R_{\lambda}^{*}\left(v\langle\mu\rangle_{n ; q, t} / a^{\prime} ; a^{\prime}, b / a^{\prime} ; q, t ; p\right)}{R_{\lambda}^{*}\left(v\langle 0\rangle_{n ; q, t} / a^{\prime} ; a^{\prime}, b / a^{\prime} ; q, t ; p\right)},
$$

where $\lambda, \mu \in \mathscr{P}_{n}$ and $a a^{\prime}=v \sqrt{t^{n-1} b}$.

Using the elliptic interpolation functions one can define elliptic binomial coefficients as

$$
\left(\begin{array}{l}
\lambda \\
\mu
\end{array}\right)_{[a, b] ; q, t ; p}:=\frac{(p q a ; q, t ; p)_{2 \lambda^{2}}}{C_{\lambda}^{-}(p q ; q, t ; p) C_{\lambda}^{-}(t ; q, t ; p) C_{\lambda}^{+}(a ; q, t ; p) C_{\lambda}^{+}(p q a / t ; q, t ; p)}
$$




$$
\times \Delta_{\mu}^{0}\left(a / b \mid t^{n}, 1 / b ; q, t ; p\right) R_{\mu}^{*}\left(t^{1-n} a^{1 / 2}\langle\lambda\rangle_{n ; q, t} ; t^{1-n} a^{1 / 2}, b a^{-1 / 2} ; q, t ; p\right),
$$

where $2 \lambda^{2}$ is shorthand for the partition $\left(2 \lambda_{1}, 2 \lambda_{1}, 2 \lambda_{2}, 2 \lambda_{2}, \ldots\right)$ and $n$ is an integer such that $n \geqslant l(\lambda), l(\mu)$. This definition is independent of the choice of $a^{1 / 2}$ and $n$. The elliptic binomial coefficients vanish unless $\mu \subseteq \lambda$ and trivialise to 1 when $\mu=0$ (but not when $\mu=\lambda$ ). It will be convenient to also use the normalised elliptic binomial coefficients

$$
\left\langle\begin{array}{l}
\lambda \\
\mu
\end{array}\right\rangle_{[a, b]\left(v_{1}, \ldots, v_{k}\right) ; q, t ; p}:=\frac{\Delta_{\lambda}^{0}\left(a \mid b, v_{1}, \ldots, v_{k} ; q, t ; p\right)}{\Delta_{\mu}^{0}\left(a / b \mid 1 / b, v_{1}, \ldots, v_{k} ; q, t ; p\right)}\left(\begin{array}{l}
\lambda \\
\mu
\end{array}\right)_{[a, b] ; q, t ; p} .
$$

In terms of these, the elliptic $\mathrm{C}_{n}$ analogue of Jackson's summation [42, Theorem 4.1] (or [8, Equation (3.7)]) is given by

$$
\sum_{\mu} \Delta_{\mu}^{0}(a / b \mid d, e ; q, t ; p)\left\langle\begin{array}{l}
\lambda \\
\mu
\end{array}\right\rangle_{[a, b] ; q, t ; p}\left\langle\begin{array}{l}
\mu \\
\nu
\end{array}\right\rangle_{[a / b, c / b] ; q, t ; p}=\left\langle\begin{array}{l}
\lambda \\
\nu
\end{array}\right\rangle_{[a, c](b d, b e) ; q, t ; p},
$$

where $b c d e=a p q$.

We can now define the elliptic skew interpolation functions as

$$
\begin{aligned}
R_{\lambda / \nu}^{*}\left(\left[v_{1}, \ldots, v_{2 n}\right] ; a, b ; q, t ; p\right):=\sum_{\mu} & \Delta_{\mu}^{0}\left(p q / b^{2} \mid p q / b v_{1}, \ldots, p q / b v_{2 n} ; q, t ; p\right) \\
& \times\left\langle\begin{array}{c}
\lambda \\
\mu
\end{array}\right\rangle_{[a / b, a b / p q] ; q, t ; p}\left\langle\begin{array}{l}
\mu \\
\nu
\end{array}\right\rangle_{\left[p q / b^{2}, p q V / a b\right] ; q, t ; p},
\end{aligned}
$$

where $V:=v_{1} \cdots v_{2 n}$. Note that unlike the $\mathrm{BC}_{n}$-symmetric interpolation functions the skew interpolation functions are $\mathfrak{S}_{2 n}$-symmetric. The rationale for using plethystic brackets around the $v_{i}$ is that in the $p \rightarrow 0$ limit they relate to the variables $x_{i}$ of the $\mathrm{BC}_{n}$-symmetric interpolation functions via the plethystic substitution

$$
x_{i}+x_{i}^{-1} \mapsto \frac{t^{1 / 2}\left(v_{2 i-1}^{-1}+v_{2 i}^{-1}-v_{2 i-1}-v_{2 i}\right)}{1-t}
$$

for all $1 \leqslant i \leqslant n$. In particular, for real $\alpha, \beta$ such that $0<\alpha<\beta<1$ and $\alpha+\beta<1$,

$$
\begin{aligned}
\lim _{p \rightarrow 0} p^{\alpha|\lambda|} R_{\lambda / 0}^{*}\left(\left[t^{1 / 2}\left(p^{-\alpha} x_{1}\right)^{ \pm}, \ldots,\right.\right. & \left.\left.t^{1 / 2}\left(p^{-\alpha} x_{n}\right)^{ \pm}, p^{-\alpha} t^{-1 / 2} c, p^{\alpha} t^{1 / 2} / d\right] ; a, p^{\beta} b ; q, t ; p\right) \\
= & \left(-a t^{-1 / 2}\right)^{|\lambda|} q^{n\left(\lambda^{\prime}\right)} t^{-2 n(\lambda)} c_{\lambda}(q, t) P_{\lambda}\left(\left[X+\frac{d-c}{1-t}\right] ; q, t\right),
\end{aligned}
$$

where $X:=x_{1}+\cdots+x_{n}$.

By (6.6) it immediately follows that

$$
R_{\lambda / \nu}^{*}\left(\left[v_{1}, v_{2}\right] ; a, b ; q, t ; p\right)=\left\langle\begin{array}{l}
\lambda \\
\nu
\end{array}\right\rangle_{\left[a / b, v_{1} v_{2}\right]\left(a / v_{1}, a / v_{2}\right) ; q, t ; p} .
$$

The skew interpolation functions further satisfy the branching rule

$$
\begin{aligned}
& R_{\lambda / \nu}^{*}\left(\left[v_{1}, \ldots, v_{2 n}, w_{1}, \ldots, w_{2 m}\right] ; a, b ; q, t ; p\right) \\
& \quad=\sum_{\mu} R_{\lambda / \mu}^{*}\left(\left[v_{1}, \ldots, v_{2 n}\right] ; a, b ; q, t ; p\right) R_{\mu / \nu}^{*}\left(\left[w_{1}, \ldots, w_{2 m}\right] ; a / V, b ; q, t ; p\right),
\end{aligned}
$$

and for $\nu=0$ generalise the $\mathrm{BC}_{n}$-symmetric interpolation functions

$$
R_{\lambda / 0}^{*}\left(\left[t^{1 / 2} x_{1}^{ \pm}, \ldots, t^{1 / 2} x_{n}^{ \pm}\right] ; t^{n-1 / 2} a, t^{1 / 2} b ; q, t ; p\right)
$$




$$
= \begin{cases}\Delta_{\lambda}^{0}\left(t^{n-1} a / b \mid t^{n} ; q, t ; p\right) R_{\lambda}^{*}\left(x_{1}, \ldots, x_{n} ; a, b ; q, t ; p\right) & \text { for } \lambda \in \mathscr{P}_{n}, \\ 0 & \text { otherwise }\end{cases}
$$

where we recall the convention (1.23).

The elliptic Selberg integral (6.1) is $p, q$-symmetric. In order to maintain this symmetry in the AFLT case, we need to generalise all of the above elliptic functions. Let $\boldsymbol{\lambda}=\left(\lambda^{(1)}, \lambda^{(2)}\right)$ and $\boldsymbol{\mu}=\left(\mu^{(1)}, \mu^{(2)}\right)$ be bipartitions. Then

$$
\begin{aligned}
R_{\boldsymbol{\mu}}^{*}(a, b ; t ; p, q) & :=R_{\mu^{(1)}}^{*}(a, b ; q, t ; p) R_{\mu^{(2)}}^{*}(a, b ; p, t ; q) \\
R_{\lambda / \boldsymbol{\mu}}^{*}(a, b ; t ; p, q) & :=R_{\lambda^{(1)} / \mu^{(1)}}^{*}(a, b ; q, t ; p) R_{\lambda^{(2)} / \mu^{(2)}}^{*}(a, b ; p, t ; q) .
\end{aligned}
$$

In much the same way we define $\Delta_{\boldsymbol{\lambda}}^{0}\left(a \mid b_{1}, \ldots, b_{k} ; t ; p, q\right)$ and $\langle\underset{\mu}{\boldsymbol{\lambda}}\rangle_{[a, b]}\left(v_{1}, \ldots, v_{k}\right) ; t ; p, q$. Finally, we recall the definition of $\langle\boldsymbol{\lambda}\rangle_{n ; t ; p, q}$, the spectral vector indexed by a bipartition, from (1.24).

For the proof of the elliptic AFLT integral we require two key identities for the interpolation functions. The first of these is the following generalised elliptic Selberg integral, see [44, Theorem 9.2]. For $\boldsymbol{\lambda}, \boldsymbol{\mu} \in \mathscr{P}_{n}^{2}$ and $t, t_{1}, t_{2}, t_{3}, t_{4}, t_{5}, t_{6} \in \mathbb{C}$ such that (1.25) holds

$$
\begin{aligned}
\kappa_{n} \int_{C_{\boldsymbol{\lambda}}} & R_{\boldsymbol{\lambda}}^{*}\left(z_{1}, \ldots, z_{n} ; t_{1}, t_{2} ; t ; p, q\right) R_{\boldsymbol{\mu}}^{*}\left(z_{1}, \ldots, z_{n} ; t_{3}, t_{6} ; t ; p, q\right) \\
& \times \prod_{1 \leqslant i<j \leqslant n} \frac{\Gamma\left(t z_{i}^{ \pm} z_{j}^{ \pm} ; p, q\right)}{\Gamma\left(z_{i}^{ \pm} z_{j}^{ \pm} ; p, q\right)} \prod_{i=1}^{n} \frac{\prod_{r=1}^{6} \Gamma\left(t_{r} z_{i}^{ \pm} ; p, q\right)}{\Gamma\left(z_{i}^{ \pm 2} ; p, q\right)} \frac{\mathrm{d} z_{1}}{z_{1}} \cdots \frac{\mathrm{d} z_{n}}{z_{n}} \\
= & \prod_{i=1}^{n}\left(\Gamma\left(t^{i} ; p, q\right) \prod_{1 \leqslant r<s \leqslant 6} \Gamma\left(t^{i-1} t_{r} t_{s} ; p, q\right)\right) \\
& \times \Delta_{\boldsymbol{\lambda}}^{0}\left(t^{n-1} t_{1} / t_{2} \mid t^{n-1} t_{1} t_{4}, t^{n-1} t_{1} t_{5} ; t ; p, q\right) \Delta_{\boldsymbol{\mu}}^{0}\left(t^{n-1} t_{3} / t_{6} \mid t^{n-1} t_{3} t_{4}, t^{n-1} t_{3} t_{5} ; t ; p, q\right) \\
& \times R_{\boldsymbol{\lambda}}^{*}\left(t_{3}\langle\mathbf{0}\rangle_{n ; t ; p, q} / \zeta^{\prime} ; t_{1} \zeta^{\prime}, t_{2} \zeta^{\prime} ; t ; p, q\right) R_{\boldsymbol{\mu}}^{*}\left(t_{1}\langle\boldsymbol{\lambda}\rangle_{n ; t ; p, q} / \zeta ; t_{3} \zeta, t_{6} \zeta ; t ; p, q\right),
\end{aligned}
$$

where $\zeta:=\sqrt{t^{n-1} t_{1} t_{2}}$ and $\zeta^{\prime}:=\sqrt{t^{n-1} t_{3} t_{6}}$, and where $C_{\boldsymbol{\lambda} \mu}$ is a deformation of $\mathbb{T}^{n}$ separating sequences of poles of the integrand tending to zero from sequences of poles tending to infinity. Note that by the evaluation symmetry (6.4) this result is invariant under the simultaneous substitution $\left(\boldsymbol{\lambda}, \boldsymbol{\mu}, t_{1}, t_{2}, t_{3}, t_{6}\right) \mapsto\left(\boldsymbol{\mu}, \boldsymbol{\lambda}, t_{3}, t_{6}, t_{1}, t_{2}\right)$. We also note that for $\boldsymbol{\mu}=0$ the above integral may be viewed as an elliptic analogue of Kadell's integral.

The second key result we need is the connection coefficient identity [42, Corollary 4.14]

$$
R_{\boldsymbol{\lambda}}^{*}\left(x_{1}, \ldots, x_{n} ; a, b ; t ; p, q\right)=\sum_{\boldsymbol{\mu}}\left\langle\begin{array}{l}
\boldsymbol{\lambda} \\
\boldsymbol{\mu}
\end{array}\right\rangle_{\left[t^{n-1} a / b, a / a^{\prime}\right]\left(t^{n-1} a a^{\prime}\right) ; t ; p, q} R_{\boldsymbol{\mu}}^{*}\left(x_{1}, \ldots, x_{n} ; a^{\prime}, b ; t ; p, q\right),
$$

where $\boldsymbol{\lambda} \in \mathscr{P}_{n}^{2}$.

6.3. Proof of Theorem 1.4. Denote the elliptic AFLT integral by $I_{\lambda \mu}$. As a first step towards proving the theorem we apply the branching rule (6.9) to expand $R_{\boldsymbol{\mu} / \mathbf{0}}^{*}$ as

$$
R_{\boldsymbol{\mu} / \mathbf{0}}^{*}\left(\left[t^{1 / 2} z_{1}^{ \pm}, \ldots, t^{1 / 2} z_{n}^{ \pm}, t^{-1 / 2} t_{4}, t^{-1 / 2} t_{5}\right] ; t^{n-3 / 2} t_{3} t_{4} t_{5}, t^{1 / 2} t_{6} ; t ; p, q\right)
$$




$$
\begin{aligned}
=\sum_{\boldsymbol{\nu} \subseteq \boldsymbol{\mu}} & R_{\boldsymbol{\mu} / \boldsymbol{\nu}}^{*}\left(\left[t^{-1 / 2} t_{4}, t^{-1 / 2} t_{5}\right] ; t^{n-3 / 2} t_{3} t_{4} t_{5}, t^{1 / 2} t_{6} ; t ; p, q\right) \\
& \times R_{\boldsymbol{\nu} / \mathbf{0}}^{*}\left(\left[t^{1 / 2} z_{1}^{ \pm}, \ldots, t^{1 / 2} z_{n}^{ \pm}\right] ; t^{n-1 / 2} t_{3}, t^{1 / 2} t_{6} ; t ; p, q\right) .
\end{aligned}
$$

By (6.8) and (6.10) this may be further rewritten as

$$
\begin{aligned}
& R_{\boldsymbol{\mu} / \mathbf{0}}^{*}( {\left.\left[t^{1 / 2} z_{1}^{ \pm}, \ldots, t^{1 / 2} z_{n}^{ \pm}, t^{-1 / 2} t_{4}, t^{-1 / 2} t_{5}\right] ; t^{n-3 / 2} t_{3} t_{4} t_{5}, t^{1 / 2} t_{6} ; t ; p, q\right) } \\
&=\sum_{\boldsymbol{\nu} \in \mathscr{P}_{n}^{2}} \Delta_{\boldsymbol{\nu}}^{0}\left(t^{n-1} t_{3} / t_{6} \mid t^{n} ; t ; p, q\right)\left\langle\begin{array}{c}
\boldsymbol{\mu} \\
\boldsymbol{\nu}
\end{array}\right\rangle_{\left[t^{n-2} t_{3} t_{4} t_{5} / t_{6}, t_{4} t_{5} / t\right]\left(t^{n-1} t_{3} t_{4}, t^{n-1} t_{3} t_{5}\right) ; t ; p, q} \\
& \quad \times R_{\boldsymbol{\nu}}^{*}\left(z_{1}, \ldots, z_{n} ; t_{3}, t_{6} ; t ; p, q\right) .
\end{aligned}
$$

If we substitute this into the elliptic AFLT integral, rewrite $R_{\boldsymbol{\lambda} / \mathbf{0}}^{*}$ in terms of $R_{\boldsymbol{\lambda}}^{*}$ using (6.10) and then interchange the order of the sum and integral, we obtain exactly the integral (6.11) (with $\boldsymbol{\mu} \mapsto \boldsymbol{\nu})$ in the summand. Hence

$$
\begin{aligned}
I_{\boldsymbol{\lambda} \boldsymbol{\mu}}= & S_{n}\left(t_{1}, t_{2}, t_{3}, t_{4}, t_{5}, t_{6} ; t ; p, q\right) \\
& \times \Delta_{\boldsymbol{\lambda}}^{0}\left(t^{n-1} t_{1} / t_{2} \mid t^{n}, t^{n-1} t_{1} t_{4}, t^{n-1} t_{1} t_{5} ; t ; p, q\right) R_{\boldsymbol{\lambda}}^{*}\left(t_{3}\langle\mathbf{0}\rangle_{n ; t ; p, q} / \zeta^{\prime} ; t_{1} \zeta^{\prime}, t_{2} \zeta^{\prime} ; t ; p, q\right) \\
& \times \sum_{\boldsymbol{\nu} \in \mathscr{P}_{n}^{2}} \Delta_{\nu}^{0}\left(t^{n-1} t_{3} / t_{6} \mid t^{n}, t^{n-1} t_{3} t_{4}, t^{n-1} t_{3} t_{5} ; t ; p, q\right) \\
& \quad \times\left\langle\begin{array}{c}
\boldsymbol{\mu} \\
\boldsymbol{\nu}
\end{array}\right\rangle_{\left[t^{n-2} t_{3} t_{4} t_{5} / t_{6}, t_{4} t_{5} / t\right]\left(t^{n-1} t_{3} t_{4}, t^{n-1} t_{3} t_{5}\right) ; t ; p, q} R_{\boldsymbol{\nu}}^{*}\left(t_{1}\langle\boldsymbol{\lambda}\rangle_{n ; t ; p, q} / \zeta ; t_{3} \zeta, t_{6} \zeta ; t ; p, q\right),
\end{aligned}
$$

where, as before, $\zeta:=\sqrt{t^{n-1} t_{1} t_{2}}$ and $\zeta^{\prime}:=\sqrt{t^{n-1} t_{3} t_{6}}$. By (1.25), (2.7) and (6.5)

$$
\begin{aligned}
& \Delta_{\nu}^{0}\left(t^{n-1} t_{3} / t_{6} \mid t^{n}, t^{n-1} t_{3} t_{4}, t^{n-1} t_{3} t_{5} ; t ; p, q\right)\left\langle\begin{array}{l}
\boldsymbol{\mu} \\
\boldsymbol{\nu}
\end{array}\right\rangle_{\left[t^{n-2} t_{3} t_{4} t_{5} / t_{6}, t_{4} t_{5} / t\right]\left(t^{n-1} t_{3} t_{4}, t^{n-1} t_{3} t_{5}\right) ; t ; p, q} \\
&=\Delta_{\boldsymbol{\mu}}^{0}\left(t^{n-2} t_{3} t_{4} t_{5} / t_{6} \mid t^{n-1} t_{3} t_{4}, t^{n-1} t_{3} t_{5}, t^{n-1} t_{4} t_{5} ; t ; p, q\right)\left\langle\begin{array}{l}
\boldsymbol{\mu} \\
\boldsymbol{\nu}
\end{array}\right\rangle_{\left[t^{n-2} t_{3} t_{4} t_{5} / t_{6}, t_{4} t_{5} / t\right]\left(p q t_{3} / t t_{6}\right) ; t ; p, q} .
\end{aligned}
$$

As a result,

$$
\begin{aligned}
I_{\boldsymbol{\lambda} \boldsymbol{\mu}}= & S_{n}\left(t_{1}, t_{2}, t_{3}, t_{4}, t_{5}, t_{6} ; t ; p, q\right) \\
& \times \Delta_{\boldsymbol{\lambda}}^{0}\left(t^{n-1} t_{1} / t_{2} \mid t^{n}, t^{n-1} t_{1} t_{4}, t^{n-1} t_{1} t_{5} ; t ; p, q\right) R_{\boldsymbol{\lambda}}^{*}\left(t_{3}\langle\mathbf{0}\rangle_{n ; t ; p, q} / \zeta^{\prime} ; t_{1} \zeta^{\prime}, t_{2} \zeta^{\prime} ; t ; p, q\right) \\
& \times \Delta_{\boldsymbol{\mu}}^{0}\left(t^{n-2} t_{3} t_{4} t_{5} / t_{6} \mid t^{n-1} t_{3} t_{4}, t^{n-1} t_{3} t_{5}, t^{n-1} t_{4} t_{5} ; t ; p, q\right) \\
& \times \sum_{\boldsymbol{\nu} \in \mathscr{P}_{n}^{2}}\left\langle\begin{array}{c}
\boldsymbol{\mu} \\
\boldsymbol{\nu}
\end{array}\right\rangle_{\left[t^{n-2} t_{3} t_{4} t_{5} / t_{6}, t_{4} t_{5} / t\right]\left(p q t_{3} / t t_{6}\right) ; t ; p, q} R_{\boldsymbol{\nu}}^{*}\left(t_{1}\langle\boldsymbol{\lambda}\rangle_{n ; t ; p, q} / \zeta ; t_{3} \zeta, t_{6} \zeta ; t ; p, q\right) .
\end{aligned}
$$

The sum over $\nu$ can be carried out by (6.12) with $\left(a, a^{\prime}, b\right) \mapsto\left(t_{3} t_{4} t_{5} \zeta / t, t_{3} \zeta, t_{6} \zeta\right)$, so that

$$
\begin{aligned}
I_{\boldsymbol{\lambda} \boldsymbol{\mu}}= & S_{n}\left(t_{1}, t_{2}, t_{3}, t_{4}, t_{5}, t_{6} ; t ; p, q\right) \\
& \times \Delta_{\boldsymbol{\lambda}}^{0}\left(t^{n-1} t_{1} / t_{2} \mid t^{n}, t^{n-1} t_{1} t_{4}, t^{n-1} t_{1} t_{5} ; t ; p, q\right) R_{\boldsymbol{\lambda}}^{*}\left(t_{3}\langle\mathbf{0}\rangle_{n ; t ; p, q} / \zeta^{\prime} ; t_{1} \zeta^{\prime}, t_{2} \zeta^{\prime} ; t ; p, q\right)
\end{aligned}
$$




$$
\begin{aligned}
& \times \Delta_{\boldsymbol{\mu}}^{0}\left(t^{n-2} t_{3} t_{4} t_{5} / t_{6} \mid t^{n-1} t_{3} t_{4}, t^{n-1} t_{3} t_{5}, t^{n-1} t_{4} t_{5} ; t ; p, q\right) \\
& \times R_{\boldsymbol{\mu}}^{*}\left(t_{1}\langle\boldsymbol{\lambda}\rangle_{n ; t ; p, q} / \zeta ; t_{3} t_{4} t_{5} \zeta / t, t_{6} \zeta ; t ; p, q\right) .
\end{aligned}
$$

We finally observe that

$$
t^{n}\left(t_{3} t_{4} t_{5} \zeta / t\right)\left(t_{6} \zeta\right)=t^{2 n-2} t_{1} t_{2} t_{3} t_{4} t_{5} t_{6}=p q
$$

so that the interpolation function in the last line is of Cauchy type and hence factors by (6.2). Thus

$$
\begin{aligned}
I_{\boldsymbol{\lambda} \boldsymbol{\mu}}= & S_{n}\left(t_{1}, t_{2}, t_{3}, t_{4}, t_{5}, t_{6} ; t ; p, q\right) \\
& \times \Delta_{\boldsymbol{\lambda}}^{0}\left(t^{n-1} t_{1} / t_{2} \mid t^{n}, t^{n-1} t_{1} t_{4}, t^{n-1} t_{1} t_{5} ; t ; p, q\right) R_{\boldsymbol{\lambda}}^{*}\left(t_{3}\langle\mathbf{0}\rangle_{n ; t ; p, q} / \zeta^{\prime} ; t_{1} \zeta^{\prime}, t_{2} \zeta^{\prime} ; t ; p, q\right) \\
& \times \Delta_{\boldsymbol{\mu}}^{0}\left(t^{n-2} t_{3} t_{4} t_{5} / t_{6} \mid t^{n-1} t_{3} t_{4}, t^{n-1} t_{3} t_{5}, t^{n-1} t_{4} t_{5} ; t ; p, q\right) \\
& \times \frac{\Delta_{\boldsymbol{\mu}}^{0}\left(t^{n-2} t_{3} t_{4} t_{5} / t_{6} \mid t^{n-2} t_{1} t_{3} t_{4} t_{5}\langle\boldsymbol{\lambda}\rangle_{n ; t ; p, q}\right)}{\Delta_{\boldsymbol{\mu}}^{0}\left(t^{n-2} t_{3} t_{4} t_{5} / t_{6} \mid t^{n-1} t_{1} t_{3} t_{4} t_{5}\langle\boldsymbol{\lambda}\rangle_{n ; t ; p, q}\right)}
\end{aligned}
$$

where we have again used (1.25) and (2.7). Using the principal specialisation formula (6.3) completes the proof.

6.4. Proof of Corollary 1.5. Throughout the proof we used condensed notation for theta and elliptic gamma functions:

$$
\begin{aligned}
\theta\left(z_{1}, \ldots, z_{k} ; p\right) & =\theta\left(z_{1} ; p\right) \cdots \theta\left(z_{k} ; p\right) \\
\Gamma\left(z_{1}, \ldots, z_{k} ; p, q\right) & =\Gamma\left(z_{1} ; p, q\right) \cdots \Gamma\left(z_{k} ; p, q\right) .
\end{aligned}
$$

Denote the integral identity obtained from $(1.26)$ by restricting $\boldsymbol{\lambda}$ and $\boldsymbol{\mu}$ to $\boldsymbol{\lambda}=(\lambda, 0)$ and $\boldsymbol{\mu}=(\mu, 0)$ as

$$
\mathscr{L}_{\lambda, \mu}\left(t_{1}, \ldots, t_{6} ; q, t ; p\right)=\mathscr{R}_{\lambda, \mu}\left(t_{1}, \ldots, t_{6} ; q, t ; p\right)
$$

where $t^{2 n-2} t_{1} t_{2} t_{3} t_{4} t_{5} t_{6}=p q$. In order to take the $p \rightarrow 0$ limit we adopt the symmetry breaking procedure of [43] and multiply the integrand of $\mathscr{L}_{\lambda, \mu}$ by [42, Corollary 1.2]

$$
1=\sum_{\sigma \in\{ \pm 1\}^{n}} \prod_{i=1}^{n} \frac{\theta\left(t_{1} z_{i}^{\sigma_{i}}, t_{3} z_{i}^{\sigma_{i}}, t_{4} z_{i}^{\sigma_{i}}, t^{n-1} t_{1} t_{3} t_{4} z_{i}^{-\sigma_{i}} ; q\right)}{\theta\left(z_{i}^{2 \sigma_{i}}, t^{i-1} t_{1} t_{3}, t^{i-1} t_{1} t_{4}, t^{i-1} t_{3} t_{4} ; q\right)} \prod_{1 \leqslant i<j \leqslant n} \frac{\theta\left(t z_{i}^{\sigma_{i}} z_{j}^{\sigma_{j}} ; q\right)}{\theta\left(z_{i}^{\sigma_{i}} z_{j}^{\sigma_{j}} ; q\right)}
$$

By $\mathrm{BC}_{n}$ symmetry, all $2^{n}$ integrals that arise are identical. Using $\Gamma(p z ; p, q)=\theta(z ; q) \Gamma(z ; p, q)$ this yields

$$
\begin{aligned}
\mathscr{L}_{\lambda, \mu}\left(t_{1}, \ldots,\right. & \left.t_{6} ; q, t ; p\right) \\
=2^{n} \kappa_{n} \int & R_{\lambda / 0}^{*}\left(\left[t^{1 / 2} z^{ \pm}\right] ; t^{n-1 / 2} t_{1}, t^{1 / 2} t_{2} ; q, t ; p\right) \\
& \times R_{\mu / 0}^{*}\left(\left[t^{1 / 2} z^{ \pm}, t^{-1 / 2} t_{4}, t^{-1 / 2} t_{5}\right] ; t^{n-3 / 2} t_{3} t_{4} t_{5}, t^{1 / 2} t_{6} ; q, t ; p\right) \\
& \times \prod_{1 \leqslant i<j \leqslant n} \frac{\Gamma\left(p t z_{i} z_{j}, t / z_{i} z_{j}, t\left(z_{i} / z_{j}\right)^{ \pm 1} ; p, q\right)}{\Gamma\left(p z_{i} z_{j}, 1 / z_{i} z_{j},\left(z_{i} / z_{j}\right)^{ \pm 1} ; p, q\right)} \\
& \times \prod_{i=1}^{n} \frac{\theta\left(t^{n-1} t_{1} t_{3} t_{4} / z_{i} ; q\right) \prod_{r \in I} \Gamma\left(p t_{r} z_{i}, t_{r} / z_{i} ; p, q\right) \prod_{r \in J} \Gamma\left(t_{r} z_{i}^{ \pm} ; p, q\right)}{\theta\left(t^{i-1} t_{1} t_{3}, t^{i-1} t_{1} t_{4}, t^{i-1} t_{3} t_{4} ; q\right) \Gamma\left(p z_{i}^{2}, z_{i}^{-2} ; p, q\right)} \frac{\mathrm{d} z}{z}
\end{aligned}
$$


where $I:=\{1,3,4\}, J:=\{2,5,6\}, z=\left(z_{1}, \ldots, z_{n}\right)$ and $\mathrm{d} z / z:=\left(\mathrm{d} z_{1} / z_{1}\right) \cdots\left(\mathrm{d} z_{n} / z_{n}\right)$. We now scale the parameters as

$$
\left(t_{1}, t_{2}, t_{3}, t_{4}, t_{5}, t_{6}\right) \mapsto\left(t_{1}, p^{1 / 2} t_{2}, t_{3}, p^{-1 / 4} t_{4}, p^{1 / 4} t_{5}, p^{1 / 2} t_{6}\right),
$$

resulting in the $p$-independent balancing condition $t^{2 n-2} t_{1} t_{2} t_{3} t_{4} t_{5} t_{6}=q$. We can now also scale the integration contour by a factor of $p^{-1 / 4}$ without passing over any poles. After also replacing $z_{i} \mapsto p^{-1 / 4} z_{i}$, all elliptic gamma functions in the resulting integral have arguments scaling as $p^{\gamma}$ for $\gamma \in[0,1]$. Then taking the $p \rightarrow 0$ limit using (6.7) (with $\alpha=1 / 4$ and $\beta=1 / 2$ ) and finally replacing $z \mapsto z / t_{5}$, we obtain

$$
\begin{aligned}
\lim _{p \rightarrow 0}( & \left.p^{(|\lambda|+|\mu|) / 4} \mathscr{L}_{\lambda, \mu}\left(t_{1}, p^{1 / 2} t_{2}, t_{3}, p^{-1 / 4} t_{4}, p^{1 / 4} t_{5}, p^{1 / 2} t_{6}\right) \prod_{i=1}^{n} \theta\left(p^{-1 / 4} t^{i-1} t_{1} t_{4}, p^{-1 / 4} t^{i-1} t_{3} t_{4} ; q\right)\right) \\
= & \left(-t^{n-1} t_{1} / t_{5}\right)^{|\lambda|}\left(-t^{n-2} t_{3} t_{4}\right)^{|\mu|} q^{n\left(\lambda^{\prime}\right)+n\left(\mu^{\prime}\right)} t^{-2 n(\lambda)-2 n(\mu)} \\
& \times c_{\lambda}(q, t) c_{\mu}(q, t) \frac{(q ; q)_{\infty}^{n}}{(t ; q)_{\infty}^{n}} \prod_{i=1}^{n} \frac{1}{\theta\left(t^{i-1} t_{1} t_{3} ; q\right)} \\
& \times \frac{1}{n !(2 \pi \mathrm{i})^{n}} \int P_{\lambda}(z ; q, t) P_{\mu}\left(\left[z+\frac{t-t_{4} t_{5}}{1-t}\right] ; q, t\right) \\
& \times \prod_{i=1}^{n} \frac{\theta\left(t^{n-1} t_{1} t_{3} t_{4} t_{5} / z_{i} ; q\right)}{\left(t_{4} t_{5} / z_{i}, z_{i} ; q\right)_{\infty}} \prod_{1 \leqslant i<j \leqslant n} \frac{\left(z_{i} / z_{j}, z_{j} / z_{i} ; q\right)_{\infty}}{\left(t z_{i} / z_{j}, t z_{j} / z_{i} ; q\right)_{\infty}} \frac{\mathrm{d} z}{z},
\end{aligned}
$$

where the contour can be chosen as $\mathbb{T}^{n}$ provided $\left|t_{4} t_{5}\right|<1$. Since the limit of the right-hand side of (1.26) is given by

$$
\begin{aligned}
\lim _{p \rightarrow 0}( & \left.p^{(|\lambda|+|\mu|) / 4} \mathscr{R}_{\lambda, \mu}\left(t_{1}, p^{1 / 2} t_{2}, t_{3}, p^{-1 / 4} t_{4}, p^{1 / 4} t_{5}, p^{1 / 2} t_{6}\right) \prod_{i=1}^{n} \theta\left(p^{-1 / 4} t^{i-1} t_{1} t_{4}, p^{-1 / 4} t^{i-1} t_{3} t_{4} ; q\right)\right) \\
= & \left(-t^{n-1} t_{1} t_{4}\right)^{|\lambda|}\left(-t^{n-1} t_{3} t_{4}\right)^{|\mu|} q^{n\left(\lambda^{\prime}\right)+n\left(\mu^{\prime}\right)} t^{-n(\lambda)-n(\mu)} \\
& \times \frac{\left(t^{n}, t^{n-1} t_{1} t_{3} ; q, t\right)_{\lambda}\left(t^{n-1} t_{4} t_{5} ; q, t\right)_{\mu}}{\left(t^{2 n-m-2} t_{1} t_{3} t_{4} t_{5} ; q, t\right)_{\lambda}} \prod_{i=1}^{n} \frac{\left(t^{2 n-i-1} t_{1} t_{3} t_{4} t_{5} ; q\right)_{\infty}}{\left(t^{i}, t^{i-1} t_{1} t_{3}, t^{i-1} t_{4} t_{5} ; q\right)_{\infty}} \\
& \times \prod_{i=1}^{n} \prod_{j=1}^{m} \frac{\left(t^{2 n-i-j-1} t_{1} t_{3} t_{4} t_{5} ; q\right)_{\lambda_{i}+\mu_{j}}}{\left(t^{2 n-i-j} t_{1} t_{3} t_{4} t_{5} ; q\right)_{\lambda_{i}+\mu_{j}}}
\end{aligned}
$$

the claim follows with $(a, b)=\left(t^{n-1} t_{1} t_{3} t_{4} t_{5}, t_{4} t_{5}\right)$.

6.5. An equivalent form of Corollary 1.5. For functions $f, g: \mathbb{C}^{n} \rightarrow \mathbb{C}$, symmetric in the $n$ variables, define the scalar product [32, p. 372]

$$
\langle f, g\rangle_{n}^{\prime}:=\frac{1}{n !(2 \pi \mathrm{i})^{n}} \int_{\mathbb{T}^{n}} f(z) g\left(z^{-1}\right) \prod_{1 \leqslant i<j \leqslant n} \frac{\left(z_{i} / z_{j}, z_{j} / z_{i} ; q\right)_{\infty}}{\left(t z_{i} / z_{j}, t z_{j} / z_{i} ; q\right)_{\infty}} \frac{\mathrm{d} z_{1}}{z_{1}} \cdots \frac{\mathrm{d} z_{n}}{z_{n}} .
$$

Then, for $\lambda \in \mathscr{P}_{n}$, 32, pp. 369\&370]

$$
\left\langle P_{\lambda}(q, t), Q_{\mu}(q, t)\right\rangle_{n}^{\prime}=\delta_{\lambda, \mu} \frac{\left(t^{n} ; q, t\right)_{\lambda}}{\left(q t^{n-1} ; q, t\right)_{\lambda}} \prod_{i=1}^{n} \frac{\left(t, q t^{i-1} ; q\right)_{\infty}}{\left(q, t^{i} ; q\right)_{\infty}} .
$$

In terms of $\langle\cdot, \cdot\rangle_{n}^{\prime}$ Corollary 1.5 may be rewritten as follows. 
Corollary 6.1. For $\lambda, \mu \in \mathscr{P}$ and $a, b, q, t \in \mathbb{C}$ such that $|b|,|q|,|t|<1$,

$$
\begin{aligned}
& \left\langle P_{\lambda}(z ; q, t) \prod_{i=1}^{n} \frac{\left(a z_{i} ; q\right)_{\infty}}{\left(b z_{i} ; q\right)_{\infty}}, Q_{\mu}\left(\left[z+\frac{t-b}{1-t}\right] ; q, t\right) \prod_{i=1}^{n} \frac{\left(q z_{i} / a ; q\right)_{\infty}}{\left(z_{i} ; q\right)_{\infty}}\right\rangle_{n}^{\prime} \\
& =t^{(1-n)|\mu|} P_{\lambda}\left(\left[\frac{1-t^{n}}{1-t}\right] ; q, t\right) Q_{\mu}\left(\left[\frac{1-b t^{n-1}}{1-t}\right] ; q, t\right) \\
& \quad \times \frac{\left(q t^{m} / a ; q, t\right)_{\lambda}}{\left(b q t^{n-1} / a ; q, t\right)_{\lambda}} \prod_{i=1}^{n} \frac{\left(t, a t^{i-1}, q t^{i-1} b / a ; q\right)_{\infty}}{\left(q, t^{i}, b t^{i-1} ; q\right)_{\infty}} \prod_{i=1}^{n} \prod_{j=1}^{m} \frac{\left(q t^{j-i} / a ; q\right)_{\lambda_{i}-\mu_{j}}}{\left(q t^{j-i+1} / a ; q\right)_{\lambda_{i}-\mu_{j}}} .
\end{aligned}
$$

where $m$ is an arbitrary integer such that $m \geqslant l(\mu)$.

For $b=t$ this is equivalent to [60, Theorem 1.7], which, as shown on page 261 of that paper, is a generalisation of (6.14).

Proof. Given a partition $\lambda \subseteq\left(N^{n}\right)$, we denote the complement of $\lambda$ with respect to $\left(N^{n}\right)$ by $\hat{\lambda}$, i.e., $\hat{\lambda}=\left(N-\lambda_{n}, \ldots, N-\lambda_{1}\right)$.

In Corollary 1.5 we replace $\lambda$ by its complement with respect to $\left(N^{n}\right)$, where $N$ is an arbitrary integer such that $\lambda_{1} \leqslant N$, and also scale $a \mapsto a q^{-N}$. Using [5, Equation (4.3)]

$$
P_{\hat{\lambda}}(x ; q, t)=\left(x_{1} \cdots x_{n}\right)^{N} P_{\lambda}\left(x^{-1} ; q, t\right)
$$

and

$$
\left(a q^{-N}, q^{N+1} / a ; q\right)_{\infty}=(-a)^{N} q^{-\left(\begin{array}{c}
N+1 \\
2
\end{array}\right)}(a, q / a ; q)_{\infty}
$$

this yields

$$
\begin{aligned}
\frac{1}{n !(2 \pi \mathrm{i})^{n}} \int_{\mathbb{T}^{n}} P_{\lambda}\left(z^{-1} ; q, t\right) P_{\mu}\left(\left[z+\frac{t-b}{1-t}\right] ; q, t\right) \\
\quad \times \prod_{i=1}^{n} \frac{\left(a / z_{i}, q z_{i} / a ; q\right)_{\infty}}{\left(b / z_{i}, z_{i} ; q\right)_{\infty}} \prod_{1 \leqslant i<j \leqslant n} \frac{\left(z_{i} / z_{j}, z_{j} / z_{i} ; q\right)_{\infty}}{\left(t z_{i} / z_{j}, t z_{j} / z_{i} ; q\right)_{\infty}} \frac{\mathrm{d} z_{1}}{z_{1}} \cdots \frac{\mathrm{d} z_{n}}{z_{n}} \\
=b^{-|\lambda|} t^{|\mu|-(n-1)|\lambda|} P_{\lambda}\left(\left[\frac{1-t^{n}}{1-t}\right] ; q, t\right) P_{\mu}\left(\left[\frac{1-b t^{n-1}}{1-t}\right] ; q, t\right) \\
\quad \times \prod_{i=1}^{n} \frac{\left(t, a t^{i-m-1} q^{-\lambda_{i}}, a t^{i-n} / b, q t^{i-1} b / a ; q\right)_{\infty}}{\left(q, t^{i}, b t^{i-1}, a t^{i-n} q^{-\lambda_{i}} / b ; q\right)_{\infty}} \prod_{i=1}^{m} \frac{\left(a t^{i-j} q^{-\lambda_{i}+\mu_{j}} ; q\right)_{\infty}}{\left(a t^{i-j-1} q^{-\lambda_{i}+\mu_{j}} ; q\right)_{\infty}}
\end{aligned}
$$

which is independent of $N$. By

$$
\begin{aligned}
& \prod_{i=1}^{n} \frac{\left(a t^{i-m-1} q^{-\lambda_{i}} ; q\right)_{\infty}}{\left(a t^{i-n} q^{-\lambda_{i}} / b ; q\right)_{\infty}} \prod_{i=1}^{n} \prod_{j=1}^{m} \frac{\left(a t^{i-j} q^{-\lambda_{i}+\mu_{j}} ; q\right)_{\infty}}{\left(a t^{i-j-1} q^{-\lambda_{i}+\mu_{j}} ; q\right)_{\infty}} \\
& \quad=\left(b t^{n-1}\right)^{|\lambda|} t^{-n|\mu|} \frac{\left(q t^{m} / a ; q, t\right)_{\lambda}}{\left(b q t^{n-1} / a ; q, t\right)_{\lambda}} \prod_{i=1}^{n} \frac{\left(a t^{i-1} ; q\right)_{\infty}}{\left(a t^{i-n} / b ; q\right)_{\infty}} \prod_{i=1}^{n} \prod_{j=1}^{m} \frac{\left(q t^{j-i} / a ; q\right)_{\lambda_{i}-\mu_{j}}}{\left(q t^{j-i+1} / a ; q\right)_{\lambda_{i}-\mu_{j}}}
\end{aligned}
$$

and the substitution $z \mapsto z^{-1}$ in the integral, the claim follows. 


\section{OPEN PROBLEMS}

To conclude the paper we will discuss a number of open problems.

7.1. Generalising Theorem 1.3 to $\gamma \neq 1$. The main open problem is to generalise Theorem 1.3 to the case of Jack polynomials. Using (1.20) this theorem can be rewritten as

$$
\begin{aligned}
& \left\langle\left(\prod_{r=1}^{n} s_{\lambda^{(r)}}\left[t^{(r)}-t^{(r-1)}\right]\right) s_{\lambda^{(n+1)}}\left[t^{(n)}+\beta-1\right]\right\rangle_{\alpha_{1}, \ldots, \alpha_{n}, \beta}^{k_{1}, \ldots, k_{n}} \\
& =\left(\prod_{r=1}^{n} s_{\lambda^{(r)}}\left[k_{r}-k_{r-1}\right]\right) s_{\lambda^{(n+1)}}\left[k_{n}+\beta-1\right] \prod_{\substack{r, s=1 \\
r \neq s}}^{n+1} \prod_{i=1}^{\ell_{r}} \frac{\left(\varepsilon_{r}\left(A_{r, s}-k_{s-1}+k_{s}\right)-i+1\right)_{\lambda_{i}^{(r)}}}{\left(\varepsilon_{r}\left(A_{r, s}+\varepsilon_{s} \ell_{s}\right)-i+1\right)_{\lambda_{i}^{(r)}}} \\
& \quad \times \prod_{1 \leqslant r<s \leqslant n+1} \prod_{i=1}^{\ell_{r}} \prod_{j=1}^{\ell_{s}} \frac{\left(A_{r, s}-i+\varepsilon_{s} j+1\right)_{\lambda_{i}^{(r)}-\varepsilon_{s} \lambda_{j}^{(s)}}}{\left(A_{r, s}-i+\varepsilon_{s}(j-1)+1\right)_{\lambda_{i}^{(r)}-\varepsilon_{s} \lambda_{j}^{(s)}}},
\end{aligned}
$$

where $\ell_{1}, \ldots, \ell_{n+1}$ are arbitrary integers such that $\ell_{r} \geqslant l\left(\lambda^{(r)}\right)$ for $1 \leqslant r \leqslant n+1, \varepsilon_{1}=\cdots=\varepsilon_{n}=1$, $\varepsilon_{n+1}=-1, k_{0}:=0$ and $k_{n+1}:=1-\beta$. It is not difficult to define a function, say

$$
R_{\lambda(1), \ldots, \lambda(n+1)}^{k_{1}, \ldots, k_{n}}\left(\alpha_{1}, \ldots, \alpha_{n}, \beta ; \gamma\right)
$$

such that for $\gamma=1$ it gives the right-hand side of (7.1) and such that for $\lambda^{(1)}=\lambda, \lambda^{(2)}=\cdots=$ $\lambda^{(n)}=0$ and $\lambda^{(n+1)}=\mu$ it yields the right-hand side of (1.13). To describe this function, we generalise our earlier definition (1.21) of $A_{r}$ and $A_{r, s}$ to include $\gamma$ :

$$
A_{r}:=\alpha_{r}+\cdots+\alpha_{n}+\left(k_{r}-k_{r-1}+r\right) \gamma \quad \text { and } \quad A_{r, s}:=A_{r}-A_{s},
$$

for $1 \leqslant r, s \leqslant n+1$. Hence $A_{r, s}=-A_{r, s}$ and

$$
A_{r, s}=\alpha_{r}+\cdots+\alpha_{s-1}+\left(k_{r}-k_{r-1}-k_{s}+k_{s-1}+r-s\right) \gamma
$$

for $1 \leqslant r \leqslant s \leqslant n+1$.

Lemma 7.1. Let $A_{r, s}$ be as in (7.2), where $0=k_{0} \leqslant k_{1} \leqslant k_{2} \leqslant \cdots \leqslant k_{n}$ are integers and $k_{n+1}:=1-\beta / \gamma$. Set $\varepsilon_{1}=\cdots=\varepsilon_{n}=1$ and $\varepsilon_{n+1}=-1$, and define

$$
\begin{aligned}
& R_{\lambda^{(1)}, \ldots, \lambda^{(n+1)}}^{k_{1}, \ldots, k_{n}}\left(\alpha_{1}, \ldots, \alpha_{n}, \beta ; \gamma\right) \\
&:=\left(\prod_{r=1}^{n} P_{\lambda^{(r)}}^{(1 / \gamma)}\left[k_{r}-k_{r-1}\right]\right) P_{\lambda^{(n+1)}}^{(1 / \gamma)}\left[k_{n}+\beta / \gamma-1\right] \\
& \times \prod_{1 \leqslant r<s \leqslant n+1} \frac{\left(-\varepsilon_{s} A_{r, s}-\varepsilon_{s}\left(k_{r-1}-k_{r}\right) \gamma ; \gamma\right)_{\lambda^{(s)}}}{\left(-\varepsilon_{s} A_{r, s}+\varepsilon_{s} \ell_{r} \gamma ; \gamma\right)_{\lambda^{(s)}}} \\
& \times \prod_{1 \leqslant r<s \leqslant n}\left(\frac{\left(A_{r, s}-\left(k_{s-1}-k_{s}\right) \gamma ; \gamma\right)_{\lambda^{(r)}}}{\left(1+A_{r, s}+\left(\varepsilon_{s} \ell_{s}-1\right) \gamma ; \gamma\right)_{\lambda^{(r)}}} \prod_{i=1}^{\ell_{r}} \prod_{j=1}^{\ell_{s}} \frac{\left(1+A_{r, s}+(j-i) \gamma\right)_{\lambda_{i}^{(r)}-\lambda_{j}^{(s)}}}{\left(1+A_{r, s}+(j-i-1)_{\lambda_{i}^{(r)}-\lambda_{j}^{(s)}}\right.}\right) \\
& \times \prod_{r=1}^{n}\left(\frac{\left(A_{r, n+1}-\left(k_{n}-k_{n+1}\right) \gamma ; \gamma\right)_{\lambda^{(r)}}}{\left(A_{r, n+1}-\ell_{n+1} \gamma ; \gamma\right)_{\lambda(r)}} \prod_{i=1}^{\ell_{n+1}} \frac{\left(A_{r, n+1}-(i+j-1) \gamma\right)_{\lambda_{i}^{(r)}+\lambda_{j}^{(n+1)}}}{\left(A_{r, n+1}-(i+j-2) \gamma\right)_{\lambda_{i}^{(r)}+\lambda_{j}^{(n+1)}}}\right),
\end{aligned}
$$


where $\ell_{1}, \ldots, \ell_{n+1}$ are arbitrary integers such that $\ell_{r} \geqslant l\left(\lambda^{(r)}\right)$ and $\ell_{1} \leqslant k_{1}$. Then

$$
R_{\lambda(1), \ldots, \lambda(n+1)}^{k_{1}, \ldots, k_{n}}\left(\alpha_{1}, \ldots, \alpha_{n}, \beta ; \gamma\right)
$$

is well-defined (i.e., independent of the choice of the $\ell_{r}$ ),

$$
R_{\lambda^{(1)}, \ldots, \lambda(n+1)}^{0, k_{2}, \ldots, k_{n}}\left(\alpha_{1}, \ldots, \alpha_{n}, \beta ; \gamma\right)= \begin{cases}R_{\lambda^{(2)}, \ldots, \lambda(n+1)}^{k_{2}, \ldots, k_{n}}\left(\alpha_{2}, \ldots, \alpha_{n}, \beta ; \gamma\right) & \text { if } \lambda^{(1)}=0 \\ 0 & \text { otherwise }\end{cases}
$$

and

$$
R_{\lambda, \underbrace{0, \ldots, 0, \mu}_{n-1 \text { times }}}^{k_{1}, \ldots, k_{n}}\left(\alpha_{1}, \ldots, \alpha_{n}, \beta ; \gamma\right) \quad \text { and } \quad R_{\lambda^{(1)}, \ldots, \lambda(n+1)}^{k_{1}, \ldots, k_{n}}\left(\alpha_{1}, \ldots, \alpha_{n}, \beta ; 1\right)
$$

agree with the right-hand side of the $\mathrm{A}_{n}$ AFLT integral (1.13) and the right-hand side of (7.1) respectively. Moreover, when $l\left(\lambda^{(1)}\right)<k_{1}$ and $k_{1}, \ldots, k_{n} \geqslant 1$,

$$
\begin{aligned}
& R_{\lambda^{(1)}, \ldots, \lambda^{(n+1)}}^{k_{1}, \ldots, k_{n}}\left(\alpha_{1}, \alpha_{2}, \ldots, \alpha_{n}, \beta ; \gamma\right) \\
& \quad=R_{\lambda^{(1)}, \ldots, \lambda(n+1)}^{k_{1}-1, \ldots, k_{n}-1}\left(\alpha_{1}+\gamma, \alpha_{2}, \ldots, \alpha_{n}, \beta+\gamma, \gamma\right) \prod_{s=1}^{n+1} \frac{\left(-\varepsilon_{s} A_{1, s}+\varepsilon_{s} k_{1} \gamma ; \gamma\right)_{\lambda^{(s)}}}{\left(-\varepsilon_{s} A_{1, s}+\varepsilon_{s}\left(k_{1}-1\right) \gamma ; \gamma\right)_{\lambda^{(s)}}} .
\end{aligned}
$$

We note that the assumption that $l\left(\lambda^{(1)}\right)<k_{1}$ is not actually a restriction since

$$
R_{\lambda(1), \ldots, \lambda(n+1)}^{k_{1}, \ldots, k_{n}}\left(\alpha_{1}, \ldots, \alpha_{n}, \beta ; \gamma\right)
$$

depends on $\lambda^{(1)}+\alpha_{1}$ only, where, for $m$ a scalar, $\lambda+m:=\left(\lambda_{1}+m, \lambda_{2}+m, \ldots\right)$. For $n=2$ and $\lambda^{(2)}=0$ the recursion (7.4) agrees with [11, Equation (A.15)] (provided $\left[k_{2} \gamma\right] /\left[\left(k_{2}-1\right) \gamma\right]$ in the latter is corrected to $\left.\left[k_{2} \gamma\right]_{\lambda} /\left[\left(k_{2}-1\right) \gamma\right]_{\lambda}\right)$. For $n>2$ and $\lambda^{(2)}=\cdots=\lambda^{(n)}=0$, however, (7.4) and the recursion at the bottom of page 36 of [11] are inconsistent.

Proof of Lemma 7.1. To see that the right-hand side of (7.3) is independent of the $\ell_{r}$, fix a $t$ such that $1 \leqslant t \leqslant n+1$. Then, assuming that $\lambda_{\ell_{t}}^{(t)}=0$, it follows from elementary manipulations and the use of

$$
\frac{(a)_{-n}}{(b)_{-n}}=\frac{(1-b)_{n}}{(1-a)_{n}}
$$

that the right-hand side of (7.3) reduces to the same expression with $\ell_{t} \mapsto \ell_{t}-1$.

For (7.4), write

$$
k_{n+1}=k_{n+1}(\beta ; \gamma) \quad \text { and } \quad A_{r, s}=A_{r, s}^{k_{1}, \ldots, k_{n}}\left(\alpha_{1}, \ldots, \alpha_{n}, \beta ; \gamma\right) .
$$

It is then readily checked that

$$
\begin{gathered}
k_{n+1}(\beta+\gamma ; \gamma)=k_{n+1}(\beta ; \gamma)-1 \\
A_{r, s}^{k_{1}-1, \ldots, k_{n}-1}\left(\alpha_{1}+\gamma, \alpha_{2}, \ldots, \alpha_{n}, \beta+\gamma ; \gamma\right)=A_{r, s}^{k_{1}, \ldots, k_{n}}\left(\alpha_{1}, \ldots, \alpha_{n}, \beta ; \gamma\right) .
\end{gathered}
$$

Hence, for $l\left(\lambda^{(1)}\right) \leqslant k_{1}-1$,

$$
\frac{R_{\lambda^{(1)}, \ldots, \lambda^{(n+1)}}^{k_{1}, \ldots, k_{n}}\left(\alpha_{1}, \alpha_{2}, \ldots, \alpha_{n}, \beta ; \gamma\right)}{R_{\lambda^{(1)}, \ldots, \lambda^{(n+1)}}^{k_{1}-1, \ldots, k_{n}-1}\left(\alpha_{1}+\gamma, \alpha_{2}, \ldots, \alpha_{n}, \beta+\gamma, \gamma\right)}=\frac{P_{\lambda^{(1)}}^{(1 / \gamma)}\left[k_{1}\right]}{P_{\lambda^{(1)}}^{(1 / \gamma)}\left[k_{1}-1\right]} \prod_{s=2}^{n+1} \frac{\left(-\varepsilon_{s} A_{1, s}+\varepsilon_{s} k_{1} \gamma ; \gamma\right)_{\lambda^{(s)}}}{\left(-\varepsilon_{s} A_{1, s}+\varepsilon_{s}\left(k_{1}-1\right) \gamma ; \gamma\right)_{\lambda^{(s)}}}
$$

By the specialisation formula (2.20) the recursion (17.4) follows.

The remaining claims of the lemma are immediate and left to the reader. 
An obvious guess would be that

$$
\begin{gathered}
\left\langle\left(\prod_{r=1}^{n} P_{\lambda^{(r)}}^{(1 / \gamma)}\left[t^{(r)}-t^{(r-1)}\right]\right) P_{\lambda^{(n+1)}}^{(1 / \gamma)}\left[t^{(n)}+\beta / \gamma-1\right]\right\rangle_{\alpha_{1}, \ldots, \alpha_{n}, \beta ; \gamma}^{k_{1}, \ldots, k_{n}} \\
=R_{\lambda^{(1)}, \ldots, \lambda^{(n+1)}}^{k_{1}, \ldots, k_{n}}\left(\alpha_{1}, \ldots, \alpha_{n}, \beta ; \gamma\right),
\end{gathered}
$$

but this is easily shown to be false unless $\lambda^{(2)}=\cdots=\lambda^{(n)}=0$. For example, by a direct computation using Theorem 1.2 and the Jack polynomial limit of (2.17) given by

$$
P_{(r)}^{(1 / \gamma)}[x-y]=x_{2}^{r} F_{1}\left(\begin{array}{c}
-\gamma,-r \\
1-\gamma-r
\end{array} ; \frac{y}{x}\right),
$$

it follows that for $k_{1}=\cdots=k_{n}=1$ and

$$
\left(\lambda^{(1)}, \ldots, \lambda^{(n)}, \lambda^{(n+1)}\right)=\left(\left(u_{1}\right), \ldots,\left(u_{n}\right), \mu\right)
$$

the left-hand side of (7.5) evaluates as a product of $n-1$ terminating ${ }_{3} F_{2}$ series. Specifically,

$$
\begin{aligned}
\left\langle\left(\prod_{r=1}^{n} P_{\left(u_{r}\right)}^{(1 / \gamma)}\left[t_{r}-t_{r-1}\right]\right) P_{\mu}^{(1 / \gamma)}\left[t_{n}+\beta / \gamma-1\right]\right\rangle_{\alpha_{1}-u_{1}, \ldots, \alpha_{n}-u_{n}, \beta ; \gamma}^{1, \ldots, 1} \\
=P_{\mu}^{(1 / \gamma)}[\beta / \gamma] \frac{\left(\alpha_{1}+\cdots+\alpha_{n}+\beta-n \gamma ; \gamma\right)_{\mu}}{\left(\alpha_{1}+\cdots+\alpha_{n}+\beta-(n-1) \gamma ; \gamma\right)_{\mu}} \\
\quad \times \prod_{r=1}^{n} \frac{\left(1-\alpha_{1}-\cdots-\alpha_{r}+(r-1) \gamma\right)_{u_{1}+\cdots+u_{r}}}{\left(1-\alpha_{1}-\cdots-\alpha_{r}-\beta_{r}+\left(r-\delta_{r, n}\right) \gamma\right)_{u_{1}+\cdots+u_{r}}} \\
\quad \times \prod_{r=1}^{n-1}{ }_{3} F_{2}\left(\begin{array}{l}
\left.-\gamma, \alpha_{1}+\cdots+\alpha_{r}-(r-1) \gamma,-u_{r+1} ; 1\right), \\
1-\gamma-u_{r+1}, 1+\alpha_{1}+\cdots+\alpha_{r}-r \gamma
\end{array}\right)
\end{aligned}
$$

where $t_{0}:=0$ and $\beta_{1}=\cdots=\beta_{n-1}:=1$. For $\gamma=1$ the $r$ th ${ }_{3} F_{2}$ series simplifies to $\delta_{u_{r+1}, 0}$ in accordance with the $k_{1}=\cdots=k_{n}$ case of (7.1). We do not know how to modify the product of Jack polynomials on the left of (7.5) so that equality holds.

7.2. Generalising Theorem 4.1 for $\gamma=1$. Another open problem is to generalise the $\gamma=1$ case of (4.8) to include a product of $n$ Schur functions. For $\beta_{n-1}+\beta_{n}=2$, denote by

$$
\langle\mathscr{O}\rangle_{\alpha_{1}, \ldots, \alpha_{n}, \beta_{n-1}, \beta_{n}}^{k_{1}, \ldots, k_{n}}
$$

the $\gamma=1$ case of the $\mathrm{A}_{n}$ Selberg average (4.6) (this again requires a complex integration contour). Then the problem is to extend the method of Section 5 to prove that

$$
\begin{aligned}
&\langle(\left.\left.\prod_{r=1}^{n-1} s_{\lambda^{(r)}}\left[t^{(r)}-t^{(r-1)}\right]\right) s_{\lambda^{(n)}}\left[t^{(n)}\right]\right\rangle_{\alpha_{1}, \ldots, \alpha_{n}, \beta_{n-1}, \beta_{n}}^{k_{1}, \ldots, k_{n}} \\
& \stackrel{?}{=} \prod_{r=1}^{n} \prod_{1 \leqslant i<j \leqslant \ell_{r}} \frac{\lambda_{i}^{(r)}-\lambda_{j}^{(r)}+j-i}{j-i} \prod_{1 \leqslant r<s \leqslant n-1} \prod_{i=1}^{\ell_{r}} \prod_{j=1}^{\ell_{s}} \frac{\lambda_{i}^{(r)}-\lambda_{j}^{(s)}+A_{r, s}+j-i}{A_{r, s}+j-i} \\
& \quad \times \prod_{r=1}^{n-1} \prod_{i=1}^{\ell_{r}} \prod_{j=1}^{k_{n}} \frac{A_{r, n+1}-i-j+1}{\lambda_{i}^{(r)}+\lambda_{j}^{(n)}+A_{r, n+1}-i-j+1}
\end{aligned}
$$




$$
\begin{aligned}
& \times \prod_{r, s=1}^{n-1} \prod_{i=1}^{\ell_{r}} \frac{\left(A_{r, s}-k_{s-1}+k_{s}-i+1\right)_{\lambda_{i}^{(r)}}}{\left(A_{r, s}+\ell_{s}-i+1\right)_{\lambda_{i}^{(r)}}} \prod_{r=1}^{n-1} \prod_{i=1}^{\ell_{r}} \frac{\left(A_{r, n}-k_{n-1}+k_{n}-i+1\right)_{\lambda_{i}^{(r)}}}{\left(A_{r, n}+\beta_{n-1}-i\right)_{\lambda_{i}^{(r)}}} \\
& \times \prod_{r=2}^{n-1} \prod_{i=1}^{k_{n}} \frac{\left(A_{r, n+1}+k_{r-1}-k_{r}-i+1\right)_{\lambda_{i}^{(n)}}}{\left(A_{r, n+1}-\ell_{r}-i+1\right)_{\lambda_{i}^{(n)}}} \frac{\left(A_{n, n+1}+k_{n-1}-k_{n}-i+1\right)_{\lambda_{i}^{(n)}}}{\left(A_{n, n+1}+\beta_{n}-i\right)_{\lambda_{i}^{(n)}}}
\end{aligned}
$$

where $t^{(0)}:=0, k_{0}=k_{n+1}:=0, \ell_{1}=k_{1}, \ell_{n}=k_{n}, \ell_{r}$ for $2 \leqslant r \leqslant n-1$ are arbitrary nonnegative integers such that $\ell_{r} \geqslant l\left(\lambda^{(r)}\right)$, and the $A_{r, s}$ are defined as in (1.21). The more general average

$$
\left\langle\left(\prod_{r=1}^{n} s_{\lambda^{(r)}}\left[t^{(r)}-t^{(r-1)}\right]\right) s_{\lambda^{(n)}}\left[t^{(n)}\right]\right\rangle_{\alpha_{1}, \ldots, \alpha_{n}, \beta_{n-1}, \beta_{n}}^{k_{1}, \ldots, k_{n}}
$$

appears not to have a similarly simple evaluation. For example, for $n=2$ and $\beta_{1}+\beta_{2}=\gamma+1$ it follows that

$$
\begin{aligned}
\left\langle P_{\left(u_{1}\right)}^{(1 / \gamma)}\left[t_{1}\right] P_{\left(u_{2}\right)}^{(1 / \gamma)}\left[t_{2}-t_{1}\right] P_{\left(u_{3}\right)}^{(1 / \gamma)}\left[t_{2}\right]\right\rangle_{\alpha_{1}, \alpha_{2}, \beta_{1}, \beta_{2} ; \gamma}^{1,1} & \\
= & \frac{\left(\alpha_{1}\right)_{u_{1}}\left(\alpha_{2}\right)_{u_{2}+u_{3}}\left(\alpha_{1}+\alpha_{2}-\gamma\right)_{u_{1}+u_{2}+u_{3}}}{\left(\alpha_{1}+\beta_{1}-\gamma\right)_{u_{1}}\left(\alpha_{2}+\beta_{2}-\gamma\right)_{u_{2}+u_{3}}\left(\alpha_{1}+\alpha_{2}\right)_{u_{1}+u_{2}+u_{3}}} \\
& \quad \times{ }_{4} F_{3}\left(\begin{array}{c}
-\gamma, \alpha_{1}+u_{1},-\alpha_{2}+\beta_{1}-u_{2}-u_{3},-u_{2} \\
1-\gamma-u_{2}, \alpha_{1}+\beta_{1}-\gamma+u_{1}, 1-\alpha_{2}-u_{2}-u_{3}
\end{array} ; 1\right) .
\end{aligned}
$$

For $\gamma=1$ this does not vanish when $u_{2}=0$, but instead yields the non-uniform expression

$$
\begin{aligned}
& \left\langle s_{\left(u_{1}\right)}\left[t_{1}\right] s_{\left(u_{2}\right)}\left[t_{2}-t_{1}\right] s_{\left(u_{3}\right)}\left[t_{2}\right]\right\rangle_{\alpha_{1}, \alpha_{2}, \beta_{1}, \beta_{2}}^{1,1} \\
& = \begin{cases}\frac{\left(\alpha_{1}\right)_{u_{1}}\left(\alpha_{2}\right)_{u_{3}}\left(\alpha_{1}+\alpha_{2}-1\right)}{\left(\alpha_{1}+\beta_{1}-1\right)_{u_{1}}\left(\alpha_{2}+\beta_{2}-1\right)\left(\alpha_{1}+\alpha_{2}-1+u_{1}+u_{3}\right)} & \text { if } u_{2}=0, \\
\frac{\left(\alpha_{1}\right)_{u_{1}}\left(\alpha_{2}\right)_{u_{2}+u_{3}-1}\left(\alpha_{1}+\alpha_{2}-1\right)\left(\beta_{1}-1\right)}{\left(\alpha_{1}+\beta_{1}-1\right)_{u_{1}+1}\left(\alpha_{2}+\beta_{2}-1\right)_{u_{2}+u_{3}}} & \text { if } u_{2} \geqslant 1 .\end{cases}
\end{aligned}
$$

7.3. An elliptic Selberg and AFLT integral for $\mathbf{A}_{\boldsymbol{n}}$. Theorem 1.4 gives an elliptic generalisation of the AFLT integral (1.6). This integral includes the elliptic Selberg integral as special case. Two obvious open problems are to generalise the $\mathrm{A}_{n}$ Selberg integral (1.12) to the elliptic level and to then further extend this to an elliptic analogue of the $\mathrm{A}_{n}$ AFLT integral (1.13). We intend to address these problems in a future paper [2].

\section{REFERENCES}

[1] V. A. Alba, V. A. Fateev, A. V. Litvinov and G. M. Tarnopolskiy, On combinatorial expansion of the conformal blocks arising from AGT conjecture, Lett. Math. Phys. 98 (2011), 33-64.

[2] S. P. Albion, E. M. Rains and S. O. Warnaar, Elliptic $\mathrm{A}_{n}$ Selberg integrals, in preparation.

[3] L. F. Alday, D. Gaiotto and Y. Tachikawa, Liouville correlation functions from four-dimensional gauge theories, Lett. Math. Phys. 91 (2010), 167-197.

[4] G. E. Andrews, R. Askey and R. Roy, Special Functions, Encyclopedia of Mathematics and its Applications, Vol. 71, Cambridge University Press, Cambridge, 1999.

[5] T. H. Baker and P. J. Forrester, Transformation formulas for multivariable basic hypergeometric series, Methods Appl. Anal. 6 (1999), 147-164. 
[6] F. J. van de Bult and E. M. Rains, Limits of multivariate elliptic hypergeometric biorthogonal functions, arXiv: 1110.1458 .

[7] I. Coman, E. Pomoni and J. Teschner, Trinion conformal blocks from topological strings, J. High Energy Phys. 2020, 078, $57 \mathrm{pp}$.

[8] H. Coskun and R. A. Gustafson, Well-poised Macdonald functions $W_{\lambda}$ and Jackson coefficients $\omega_{\lambda}$ on BC$C_{n}$, pp. 127-155 in Jack, Hall-Littlewood and Macdonald polynomials, Contemp. Math., 417, Amer. Math. Soc., Providence, RI, 2006.

[9] J. F. van Diejen and V. P. Spiridonov, An elliptic Macdonald-Morris conjecture and multiple modular hypergeometric sums, Math. Res. Lett. 7 (2000), 729-746.

[10] J. F. van Diejen and V. P. Spiridonov, Elliptic Selberg integrals, Internat. Math. Res. Notices 20 (2001), 10831110.

[11] V. A. Fateev and A. V. Litvinov, Integrable structure, W-symmetry and AGT relation, J. High Energy Phys. 2012, 051, 39 pp.

[12] B. Feigin, D. Fuchs, Invariant skew-symmetric differential operators on the line and Verma modules over the Virasoro algebra, Funct. Anal. Appl. 16 (1982) 114-126.

[13] R. Flume and R. Poghossian, An algorithm for the microscopic evaluation of the coefficients of the Seiberg-Witten prepotential, Int. J. Mod. Phys. A 18 (2003), 2541-2563.

[14] P. J. Forrester, Log-Gases and Random Matrices, London Math. Soc. Monographs Series, 34, Princeton University Press, Princeton, NJ, 2010.

[15] P. J. Forrester and S. O. Warnaar, The importance of the Selberg integral, Bull. Amer. Math. Soc. 45 (2008), 489-534.

[16] F. Fucito, J. F. Morales and R. Poghossian, Instantons on quivers and orientifolds, J. High Energy Phys. 2004, 037, 23 pp.

[17] G. Gasper and M. Rahman, Basic Hypergeometric Series, second edition, Encyclopedia of Mathematics and its Applications, Vol. 96, Cambridge University Press, Cambridge, 2004.

[18] J. Haglund, The q, t-Catalan Numbers and the Space of Diagonal Harmonics, Univ. Lecture Ser., vol. 41, American Mathematical Society, Providence, RI, 2008.

[19] L. K. Hua, Harmonic Analysis of Functions of Several Complex Variables in the Classical Domains, Translations of Mathematical Monographs, 6, AMS, Providence, RI, 1979.

[20] M. Isachenkov, V. Mitev and E. Pomoni, Toda 3-point functions from topological strings II, J. High Energy Phys. 2016, 066, 48 pp.

[21] M. Ito and M. Noumi, Evaluation of the $B C_{n}$ elliptic Selberg integral via the fundamental invariants, Proc. Amer. Math. Soc. 145 (2017), 689-703.

[22] H. Itoyama, T. Oota and R. Yoshioka, 2d-4d connection between $q$-Virasoro/W block at root of unity limit and instanton partition function on ALE space, Nuclear Phys. B 877 (2013), 506-537.

[23] K. W. J. Kadell, An integral for the product of two Selberg-Jack symmetric polynomials, Compositio Math. 87 (1993), 5-43.

[24] K. W. J. Kadell, The Selberg-Jack symmetric functions, Adv. Math. 130 (1997), 33-102.

[25] K. W. J. Kadell, The Schur functions for partitions with complex parts, pp. 247-270 in q-Series from a Contemporary Perspective, Contemp. Math., 254, Amer. Math. Soc., Providence, RI, 2000.

[26] J. Kaneko, q-Selberg integrals and Macdonald polynomials, Ann. Sci. École Norm. Sup. (4) 29 (1996), $583-637$.

[27] J. Kaneko, $A_{1} \Psi_{1}$ summation theorem for Macdonald polynomials, Ramanujan J. 2 (1998), 379-386.

[28] C. Krattenthaler, Advanced determinant calculus, Sém. Lothar. Combin. 42 (1999), Art. B42q, 67 pp.

[29] A. Lascoux, Symmetric functions and combinatorial operators on polynomials, CMBS Reg. Conf. Ser. Math., vol. 99, American Mathematical Society, Providence, RI, 2003.

[30] J.-G. Luque and J.-Y Thibon, Hankel hyperdeterminants and Selberg integrals, J. Phys. A 36 (2003), $5267-5292$.

[31] I. G. Macdonald, Commuting differential operators and zonal spherical functions, pp. 189-200 in Algebraic Groups, Lecture Notes in Math. 1271, Springer, Berlin, 1987.

[32] I. G. Macdonald, Symmetric Functions and Hall Polynomials, second edition, The Clarendon Press, Oxford University Press, New York, 1995.

[33] I. G. Macdonald, Hypergeometric functions II, arXiv:1309.5208.

[34] K. Mimachi and Y. Yamada, Singular vectors of the Virasoro algebra in terms of Jack symmetric polynomials, Comm. Math. Phys. 174 (1995), 447-455. 
[35] A. Mironov, A. Morozov, Sh. Shakirov and A. Smirnov, Proving AGT conjecture as HS duality: extension to five dimensions, Nuclear Phys. B 855 (2012), 128-151.

[36] Y. Nakayama, Liouville field theory: a decade after the revolution, Int. J. Mod. Phys. A 19 (2004), 2771-2930.

[37] N. A. Nekrasov, Seiberg-Witten prepotential from instanton counting, Adv. Theor. Math. Phys. 7 (2004), $831-$ 864.

[38] N. A. Nekrasov and A. Okounkov, Seiberg-Witten theory and random partitions, pp. 525-596 in The Unity of Mathematics, Progr. Math., 244, Birkhäuser Boston, Boston, MA, 2006.

[39] A. Okounkov, (Shifted) Macdonald polynomials: q-integral representation and combinatorial formula, Compositio Math. 112 (1998), 147-182.

[40] A. Okounkov, BC-type interpolation Macdonald polynomials and binomial formula for Koornwinder polynomials, Transform. Groups 3 (1998), 181-207.

[41] A. Okounkov and G. Olshanski, Shifted Jack polynomials, binomial formula, and applications, Math. Res. Lett. 4 (1997), 69-78.

[42] E. M. Rains, $B C_{n}$-symmetric abelian functions, Duke Math. J. 135 (2006), 99-180.

[43] E. M. Rains, Limits of elliptic hypergeometric integrals, Ramanujan J. 18 (2009), 257-306.

[44] E. M. Rains, Transformations of elliptic hypergeometric integrals, Ann. of Math. (2) 171 (2010), 169-243.

[45] E. M. Rains, Elliptic Littlewood identities, J. Combin. Theory Ser. A 119 (2012), 1558-1609.

[46] E. M. Rains and S. O. Warnaar, Bounded Littlewood identities, Mem. Amer. Math. Soc. 270 (2021), No. 1317, vii+115 pp.

[47] H. Rosengren, Determinantal elliptic Selberg integrals, Sém. Lothar. Combin. 81 (2020), Art. B81g, 10 pp.

[48] H. Rosengren and S. O. Warnaar, Elliptic hypergeometric functions associated with root systems, pp. 159-186 in Multivariable Special Functions, Cambridge University Press, Cambridge, 2020.

[49] S. N. M. Ruijsenaars, First order analytic difference equations and integrable quantum systems, J. Math. Phys. 38 (1997), 1069-1146.

[50] R. Sakamoto, J. Shiraishi, D. Arnaudon, L. Frappat, E. Ragoucy, Correspondence between conformal field theory and Calogero-Sutherland model, Nucl. Phys. B 704 (2005), 490-509.

[51] A. Selberg, Bemerkninger om et multipelt integral, Norsk. Mat. Tidsskr. 24 (1944), 71-78.

[52] S. Shadchin, Cubic curves from instanton counting, J. High Energy Phys. 2006, 046, 27 pp.

[53] V. P. Spiridonov, On the elliptic beta function, Russian Math. Surveys 56 (2001), 185-186.

[54] V. P. Spiridonov, Short proofs of the elliptic beta integrals, Ramanujan J. 13 (2007), 1-3.

[55] R. P. Stanley, Some combinatorial properties of Jack symmetric functions, Adv. Math. 77 (1989), $76-115$.

[56] V. Tarasov and A. Varchenko, Selberg-type integrals associated with $\mathfrak{s l}_{3}$, Lett. Math. Phys. 65, (2003), 173-185.

[57] V. Tarasov and A. Varchenko, Knizhnik-Zamolodchikov type equations, Selberg integrals and related special functions, pp. 368-401 in Multivariable Special Functions, Cambridge University Press, Cambridge, 2020.

[58] J. Teschner, Liouville theory revisited, Class. Quant. Grav. 18 (2001), R153-R222.

[59] A. Tsuchiya and Y. Kanie, Fock space representations of the Virasoro algebra. Intertwining operators, Publ. Res. Inst. Math. Sci. 22 (1986), 259-327.

[60] S. O. Warnaar, q-Selberg integrals and Macdonald polynomials, Ramanujan J. 10 (2005), 237-268.

[61] S. O. Warnaar, On the generalised Selberg integral of Richards and Zheng, Adv. in Appl. Math. 40 (2008), $212-218$.

[62] S. O. Warnaar, Bisymmetric functions, Macdonald polynomials and $\mathfrak{s l}_{3}$ basic hypergeometric series, Composito Math. 144 (2008), 271-303.

[63] S. O. Warnaar, A Selberg integral for the Lie algebra $\mathrm{A}_{n}$, Acta Math. 203 (2009), 269-304.

[64] S. O. Warnaar, The $\mathfrak{s l}_{3}$ Selberg integral, Adv. Math. 224 (2010), 499-524.

[65] S. Yanagida, Whittaker vectors of the Virasoro algebra in terms of Jack symmetric polynomial, J. Algebra 333 (2011), 273-294.

[66] H. Zhang and Y. Matsuo, Selberg integral and $\mathrm{SU}(N)$ AGT conjecture, J. High Energy Phys. 2011, 106, 38 pp. 
Fakultät für Mathematik, Universität Wien, Oskar-Morgenstern-Platz 1, A-1090, Vienna, Austria

Email address: seamus.albion@univie.ac.at

Department of Mathematics, California Institute of Technology, Pasadena, CA 91125, USA

Email address: rains@caltech.edu

School of Mathematics and Physics, The University of Queensland, Brisbane, QLD 4072, Australia

Email address: o. warnaar@maths.uq.edu.au 